\title{
Time Dependence of the Ultraviolet Radiation Field in the Local Interstellar Medium
}

\author{
Antonio Parravano ${ }^{1,2}$, David J. Hollenbach ${ }^{2}$, and Christopher F. McKee ${ }^{3}$
}

\begin{abstract}
Far Ultraviolet (FUV, $6 \mathrm{eV}<h \nu<13.6 \mathrm{eV}$ ) radiation has been recognized as the main source of heating of the neutral interstellar gas, and, as a consequence, it determines whether the thermal balance of the neutral gas results in cold $(T \sim 50-100 K)$ clouds $(\mathrm{CNM})$, warm $\left(T \sim 10^{4} K\right)$ clouds $(\mathrm{WNM})$, or a combination of the two. High FUV fields convert the neutral gas to WNM, while low fields result in CNM. The knowledge of how these fractions depend on the FUV sources (i.e. the star formation rate, the IMF, and the size distribution of associations) is a basic step in building any detailed model of the large scale behavior of the ISM and the mutual relation between the ISM and the star formation rate in a galaxy.

The sources of FUV radiation are the short-lived massive stars that generally originate in associations that form in Giant Molecular Clouds present in the galactic disk. Using McKee \& Williams' (1997) distribution of birthrates for OB associations in the Galaxy, we determine the expected behavior of the time-dependent FUV field for random positions in the local ISM. The FUV field is calculated in two bands $(912-1100 \AA$ and $912-2070 \AA)$ and at the wavelength $1400 \AA$. In terms of $U_{-17} \equiv U /\left(10^{-17} \operatorname{erg~} \mathrm{cm}^{-3} \AA^{-1}\right)$, where $U$ is the energy density of the radiation field in some band, we find (mean, median) values at the solar circle of $U_{-17}=(15.7,7.4)$ and $(14.2,7.2)$ for the $[912-1100 \AA]$ and $[912-2070 \AA]$ bands, respectively. At $1400 \AA$ we find (mean, median) values of $U_{-17}=(14.4,7.5)$. Our median value for the [912-2070 $\left.\mathrm{A}\right]$ band is $G_{0}=1.6$ times Habing's (1968) value for the radiation field at the solar circle in this band, and quite close to Draine's (1976) value, $G_{0}=1.7$. Both the latter values are based on observations of sources of FUV radiation in the solar neighborhood, so all three values are close to observed values. Due to attenuation by dust, only associations within about 500 pc contribute significantly to the energy density at a given point. Large angle scattering produces a diffuse field that is about $10 \%$ of the field produced by the sum of direct and small angle $\left(<5^{\circ}\right)$ scattering from discrete sources (the associations), as observed. At a point exposed to the median radiation field, the brightest association typically produces about $20 \%$ of the total energy density. At a
\end{abstract}

\footnotetext{
${ }^{1}$ Universidad de Los Andes, Centro De Astrofísica Teórica, Mérida 5101a, Venezuela

${ }^{2}$ NASA Ames Research Center, MS 245-3, Moffett Field, CA 94035

${ }^{3}$ Physics Department and Astronomy Department University of California at Berkeley, Berkeley, CA 94720
} 
point exposed to an above average radiation field, the brightest association produces most of the energy density. Therefore, the FUV field is asymmetric at a given point, and the asymmetry grows for higher fields.

The FUV field fluctuates with a variety of amplitudes, the larger ones being less frequent. The mean field is about twice the median field because of these fluctuations, or spikes, in the radiation field. These spikes, which last $\sim 30 \mathrm{Myr}$, are caused by the infrequent birth of nearby associations. For spikes that are significantly higher than the mean field, the time interval between spikes is $\sim 2 U_{-15}^{3 / 2}$ Gyr. We also model shorter duration spikes caused by runaway OB stars. The presence of a fluctuating heating rate created by the fluctuating FUV field converts CNM to WNM and vice versa.

Subject headings: ISM: structure — ISM: evolution — Stars: formation — Stars: mass function

\section{Introduction}

Neutral interstellar gas is the dominant mass component of the interstellar medium (ISM), and the atomic gas exists as two phases in rough pressure equilibrium: the warm neutral medium (WNM) with hydrogen densities at the solar circle of $n \sim 0.3 \mathrm{~cm}^{-3}$ and $T \simeq 8000 \mathrm{~K}$, and the cold neutral medium (CNM) with $n \sim 40 \mathrm{~cm}^{-3}$ and $T \sim 70 \mathrm{~K}$ (Wolfire et al. 1995, 2002). With sufficient shielding column density, $N>10^{20-21} \mathrm{~cm}^{-2}$, the CNM clouds harbor molecular interiors, and for $N>10^{22} \mathrm{~cm}^{-2}$ they become gravitationally bound and stars may form in their molecular interiors (e.g., McKee 1989). Most of the star formation in the Galaxy occurs in Giant Molecular Clouds (GMCs) with large masses $M \sim 10^{5-6} \mathrm{M}_{\odot}$ and columns $N \sim 10^{22} \mathrm{~cm}^{-2}$.

In spiral galaxies FUV radiation from OB stars heats, photodissociates, and controls the state of the neutral interstellar gas. High FUV fields heat CNM to WNM; low FUV fields allow WNM to cool to CNM (Wolfire et al. 1995, 2002). If, as seems likely, the formation rate of stars in galaxies depends on the mass fraction of CNM gas, then a possible feedback process occurs between stars and the ISM that may control the global rate of star formation in a galaxy (Parravano 1988, 1989, 1991; Corbelli \& Salpeter 1995). High star formation rates lead to large numbers of OB stars that create high FUV fluxes that destroy the sources of star formation. Low rates of star formation, on the other hand, may lead to enhanced growth rates of star-forming clouds and thereby to enhanced star formation rates. In this paper we focus on the calculation of the fluctuating FUV field in the local region of the Milky Way Galaxy, deferring to future papers the study of the fractional amounts of CNM and WNM produced by the FUV field, and the possible regulation of star formation rates in galaxies.

OB stars are generally born in associations that form inside GMCs present in the galactic disk. The FUV field at a given point in a galaxy then depends largely on the distribution of 
stellar masses in relatively nearby associations and on the birth-rate and size distribution of these associations. The FUV luminosity of an association is an increasing function of its size, but the birth-rate of associations decreases with size. This fact, coupled with the fact that the probability of a young association existing within a distance $r$ of a point decreases rapidly with decreasing $r$, leads to a fluctuating FUV field, with the larger fluctuations being less frequent. The abundant low luminosity or distant associations help to build an FUV background, over which is added bursts of FUV flux coming from relatively rare events (the birth of nearby large associations) that can significantly increase the FUV field for about $\sim 30$ to 50 Myr. This period corresponds to the time that an association produces stars ( $\sim 20 \mathrm{Myr}$, generally in a mode of several bursts) plus the time that early B stars, which dominate the FUV production, remain alive after the end of the star formation process in the GMC. The time variation of the FUV field at any given point in a galaxy maps directly onto the spatial variation of the FUV field in the galaxy at any instant of time: those regions very close to luminous associations will have exceptionally high FUV fluxes, whereas large volumes of space will have much lower FUV fluxes.

The presence of a time and space-dependent FUV field has a major impact on the structure of the interstellar medium. In a subsequent paper we calculate the response of interstellar gas to this fluctuating FUV field, estimate the average fractions of the gas in the CNM and WNM phases, and determine the typical time that the gas remains in each phase. The knowledge of these quantities and how they depend on the gas properties (i.e., mean density and composition) and on the FUV-sources (i.e., star formation rate SFR, initial mass function IMF, and size distribution of associations) is a basic step in building any detailed model of the large scale behavior of the ISM and the mutual relation between the ISM and the SFR.

FUV photons heat the neutral interstellar gas primarily by photoejecting energetic $(\sim 1$ eV) electrons into the gas from interstellar dust grains (Watson, 1972; Jura, 1976; de Jong, 1977; Draine, 1978) and large stable molecules like polycyclic aromatic hydrocarbons (PAHs) (d'Hendecourt and Léger, 1987; Lepp and Dalgarno, 1988; Verstraete et al., 1990; Bakes and Tielens, 1994). The relevant wavelength range for this "grain photoelectric heating" process is determined by the frequency dependence of the absorption cross sections and the photoelectric yields of grains and PAHs. Since we are interested in the neutral ISM, the Lyman threshold $(912 \AA)$ is the shortest wavelength of the relevant band. The long wavelength limit is somewhat arbitrary. It depends on the wavelength at which both the absorption cross section and the yield has fallen significantly for grains as well as for PAHs (Draine 1978; Bakes \& Tielens 1994, and references therein). In general, the band between $6 \mathrm{eV} \leq h \nu \leq 13.6 \mathrm{eV}$ (i.e. [912-2070 $\AA]$ ) has been considered as the primary band for grain photoelectric heating, and a mean value of the flux in the band is often used as a parameter to calculate the thermochemical equilibrium of the neutral gas. However, for a given total flux in the band, a change in its energy distribution can have a large effect on the photodissociation rates and even the heating rates. In particular, the main processes of $\mathrm{H}_{2}$ and $\mathrm{CO}$ destruction is photodissociation by radiation in the band [912-1100 $\AA$ ]. We therefore follow two primary bands: 
a) The band $[912-2070 \AA]$, hereafter the "FUV band". The mean energy density in this band is related to the parameter $G_{0}$ that is often used to parametrize the photoelectric dust heating rate (Tielens \& Hollenbach 1985). Let $U_{\text {band }} \equiv U\left(\right.$ band) $/ \Delta_{\text {band }}$ be the average radiation energy density per $\AA$ in the wavelength band $\Delta_{\text {band }}$. The energy density of FUV radiation is generally expressed in units of the Habing (1968) typical energy density at the solar circle averaged between $6 \mathrm{eV} \leq h \nu \leq 13.6 \mathrm{eV}$ (i.e. $[912-2070 \AA]$ ),

$$
G_{0} \equiv \frac{U_{\mathrm{FUV}}}{U_{\mathrm{FUV}}^{\mathrm{H}}}=\frac{U_{\mathrm{FUV}}}{4.6 \times 10^{-17} \mathrm{erg} \mathrm{cm}^{-3} \AA^{-1}} .
$$

If the radiation field is one-dimensional, it is convenient to express the Habing field as a flux, $c U_{\mathrm{FUV}}^{\mathrm{H}} \Delta_{\mathrm{FUV}}=1.6 \times 10^{-3} \mathrm{erg} \mathrm{cm}^{-2} \mathrm{~s}^{-1}$.

b) The band $[912-1100 \AA]$, hereafter the " $\mathbf{H}_{2}$ band". The flux in this band determines the dissociation rate of $\mathrm{H}_{2}$ and $\mathrm{CO}$. It is also useful to consider separately the contribution of this band to the photoelectic heating. Even though the $\mathrm{H}_{2}$ band covers only about $10 \%$ of the whole FUV wavelength band, both the absorption cross section and the yield of dust are significantly higher in the $\mathrm{H}_{2}$ band, so that it contributes disproportionately to the gas heating. On the other hand, the extinction in the $\mathrm{H}_{2}$ band is about twice the extinction in the FUV band, which reduces the importance of the $\mathrm{H}_{2}$ band heating.

In addition to these two rather broad bands, simulations in a $1 \AA$ band around $1400 \AA$ are performed in order to compare the averages of the time dependent FUV model with observations. Many observations have been made of the interstellar field at $1400 \AA$, although the observations are averages over bands considerably broader than $1 \AA$.

In our model, we include both direct and scattered FUV, and therefore, at a given time the local value of the FUV interstellar radiation field (hereafter FUV-ISRF) can be reasonably determined from the location of the FUV sources and the distribution and properties of the absorbing and scattering dust particles. In order to construct a time dependent model for FUV interstellar radiation we adopt: a) an initial mass function for stars in associations (§2.1); b) a stellar-mass dependence of the FUV and $\mathrm{H}_{2}$ band luminosities, and the main sequence lifetime for stars of various masses $(\S 2.2)$; c) the dependence of the birth-rate of associations on the number of stars in the association, the history of the star formation rate in an association, and the distribution in the galactic plane of the association birthplaces from McKee \& Williams (1997; hereafter MW97) (§3); and d) a vertical distribution of associations and dust (§4). We also present in $\S 4$ model simulations of the time dependence of the FUV-ISRF, together with the corresponding asymptotic values. The typical FUV-ISRF is computed as the median value in the time-sequence. We compare in $\S 5$ the mean and median values derived with the time-dependent model with previous estimations of the local field. In $\S 6$ we quantify the effect of runaway OB stars on the detailed time evolution of the FUV-ISRF. Finally, we summarize the results in $\S 7$. 


\section{Stellar Properties}

\subsection{Stellar IMF and PDMF}

Let $\mathcal{N}_{*}(>m)$ be the number of stars with masses greater than $m M_{\odot}$, and let $d \mathcal{N}_{*}(m) \equiv-d \mathcal{N}_{*}(>m)$ be the number of stars with masses between $m$ and $m+d m$. We express the present day mass function (PDMF) as $d \mathcal{N}_{*}(m) / d \ln m$, which is simply the number of stars in a logarithmic mass interval. We denote the PDMF per unit area of Galactic disk by

$$
\varsigma_{*}(m) \equiv \frac{d^{2} \mathcal{N}_{*}(m)}{d A d \ln m} .
$$

This quantity is related to the quantity $\phi_{\mathrm{ms}}$ defined by Miller \& Scalo (1979; hereafter MS79) by $\varsigma_{*}(m)=\phi_{\mathrm{ms}} \log e=0.434 \phi_{\mathrm{ms}}$.

The stellar birthrate as a function of mass - the IMF — is given by $d \dot{\mathcal{N}}_{*}(m) / d \ln m$; note that $\dot{\mathcal{N}}_{*}(m)$ includes only stellar birth, not stellar death. The IMF per unit area of Galactic disk is $\dot{\zeta}_{*}(m)$, which is related to MS79's $\xi(\log m)$ by $\int \dot{\varsigma}(m, t) d t=0.434 \xi(\log m)$. Sometimes the IMF is described by the fraction of stars above mass $m$ [e.g., $F_{n}(>m)-$ MS79], but this depends on the uncertain and possibly variable number of low-mass stars in the IMF. Since we are interested in the stars that produce the FUV radiation, we shall generally characterize the IMF in terms of quantities related to the high-mass end of the IMF.

For massive stars, the IMF can be approximated as a cut-off power law that extends up to a mass $m_{u}$ (MW97),

$$
\frac{d \dot{\mathcal{N}}_{*}(m)}{d \ln m}=\dot{\mathcal{N}}_{* u}\left(\frac{m_{u}}{m}\right)^{\Gamma} \quad\left(m \leq m_{u}\right)
$$

where $\dot{\mathcal{N}}_{* u}$ is approximately equal to the birthrate of stars with masses between $0.5 m_{u}$ and $m_{u}$ (for $\Gamma=1$ this relation is exact). Young star clusters have PDMFs that are of this form also, although the parameters $m_{u}$ and $\Gamma$ that enter this distribution may differ from those in the IMF due to stellar mass loss and, eventually, to supernovae. The birthrate of stars more massive than $m$ is

$$
\dot{\mathcal{N}}_{*}(>m)=\frac{\dot{\mathcal{N}}_{* u}}{\Gamma}\left[\left(\frac{m_{u}}{m}\right)^{\Gamma}-1\right] .
$$

In most associations, there are very few stars with masses near $m_{u}$, so it is convenient to express the IMF in terms of $\dot{\mathcal{N}}_{* h}$, the birthrate of high-mass stars (those with masses $m \geq m_{h}$ ),

$$
\frac{d \dot{\mathcal{N}}_{*}(m)}{d \ln m}=\dot{\mathcal{N}}_{* h}\left(\frac{\Gamma}{\phi_{h}}\right)\left(\frac{m_{h}}{m}\right)^{\Gamma}
$$

where $\phi_{h} \equiv 1-\left(m_{h} / m_{u}\right)^{\Gamma}$ is a numerical parameter that is of order unity; indeed, for the fiducial values of the parameters that we adopt below, $\phi_{h}=0.974$.

The value of $m_{h}$ is somewhat arbitrary; we adopt $m_{h}=8$ since most ionizing photons are produced by stars above this mass and since this is the minimum mass for core-collapse 
supernovae. The value of $\Gamma$ is also somewhat uncertain. Van Buren (1983) carried out a careful study of the high stellar mass IMF of the Galaxy, and concluded that $\Gamma=1.03$. A fit to the high-mass end of Scalo's (1986) IMF gives $\Gamma \simeq 1.5$. In a study of OB associations in the northern Milky Way, Massey, Johnson, \& DeGioia-Eastwood (1995) found a mean value $\Gamma=1.1 \pm 0.1$. In the star cluster R136 located in the LMC, Massey and Hunter (1998) found $\Gamma \simeq 1.35$. While it remains controversial whether the IMF is a universal function (Scalo 1998), there is no evidence that the variations seen in the IMF are anything but statistical in nature. We adopt a single power-law IMF with $\Gamma=1.35$ for stars more massive than $8 M_{\odot}$. As described below and in Appendix A, the probabilistic distribution used here allows for fluctuations around this power law in a given association.

The existence of an upper limit to the mass function is also controversial; for example, Massey and Hunter (1998) suggest that the apparent cutoff is due to the sparcity of very massive stars. This issue was addressed in a general way by MW97. If the distribution of a quantity $x$ can be approximated by a cutoff-power law of the form in equation (价),

$$
\mathcal{N}(>x)=\frac{\mathcal{N}_{u}}{\Gamma}\left[\left(\frac{x_{u}}{x}\right)^{\Gamma}-1\right],
$$

then the expected number of objects with $x>x_{u}$ in the absence of a physical cutoff is $\mathcal{N}_{u} / \Gamma$. If this number is large, then the cutoff must be physically significant, whereas if it is $\lesssim 1$ then it is not. In R136, Massey and Hunter (1998) find 29 stars more massive than $75 M_{\odot}$, but none more massive than $155 M_{\odot}$ (based on the stellar data of Vacca, Garmany, \& Shull 1996). Setting $m_{u}=155$ in this case, we find $\mathcal{N}_{u} / \Gamma=17.4 \gg 1$. Hence, we conclude that the upper mass limit in R136 is physically significant. In the Galaxy, it is not clear that $m_{u}$ is as large as 155 . In their survey of OB associations in the northern Milky Way, Massey et al. (1995) found only one star

more massive than $120 M_{\odot}$, whereas many more would have been expected if the IMF continued on to higher masses. We shall adopt $m_{u}=120$ here.

\subsection{Stellar Sources of FUV Radiation}

As they evolve, stars change their radii and effective temperatures and therefore their FUV luminosities. However, we are interested in the FUV radiation field from a large population of stars of different ages and in different associations, and therefore the use of a mean luminosity during the main sequence (MS) lifetime, $t_{\mathrm{ms}}$, is an adequate approximation. During the large but infrequent fluctuations of the FUV field that occur when a single nearby association dominates the radiation field, this approximation is not as good as in the quiescent period when associations of different ages contribute to the FUV field. However, even in a single association, several generations of stars can contribute to the total luminosity of the association, and the use of time-averaged FUV luminosities provides an adequate description of the total FUV luminosity of the association. Therefore, in our model the time variation of the total FUV luminosity of an 
association is due to the birth and death of its massive stars, and not to the luminosity changes in individual stars during their MS lifetime.

To compute the mean main-sequence luminosities we have used the set of stellar evolutionary tracks generally referred as the Padova tracks (Bruzual 1999, and references therein), with the stellar flux in the $\mathrm{H}_{2}$ and FUV bands kindly provided by G. Bruzual and J. Mateu (based on the library of synthetic stellar spectra compiled by Lejeune et al. 1997ab). Hereafter, we denote as $L_{\mathrm{FUV}}$ and $L_{H 2}$ the mean main-sequence luminosities in the FUV band, [912-2070 $\AA$ ], and in the $\mathrm{H}_{2}$ band, [912 - $\left.1100 \AA\right]$, respectively. For stars with solar metallicity, Figures 1a and 1b show respectively the resulting mean luminosities in the band [1100-2070 $\AA$ ] (i.e. $L_{\mathrm{FUV}}-L_{\mathrm{H} 2}$ ) and in the $\mathrm{H}_{2}$ band (i.e. $L_{H 2}$ ) as a function of the zero age main sequence stellar mass $m$ in units of $M_{\odot}$. In Figures 1a and 1b the triangles correspond to the calculated mean MS luminosities using the stellar evolutionary tracks, and the lines are the eight-segment power law used to approximate the mass dependence of the luminosity in the simulations. Table 1 shows the adopted power laws for $L_{\mathrm{FUV}}-L_{H 2}, L_{H 2}, s_{49}$ (the ionizing photon luminosity, see below), and the main sequence lifetime $t_{\mathrm{ms}}(m)$. The power law approximations for the luminosities are good to within $10 \%$, and those for the main sequence lifetime are good to within $5 \%$.

When a massive star is young and embedded or near its natal cloud, the natal cloud absorbs a fraction of the emitted radiation. Due to their shorter lifetimes, more massive stars are more affected by this obscuration. We assume that the fraction of radiation locally absorbed goes linearly from $3 / 4$ to zero in a time $t_{\text {obsc }}$. In the nearest region of massive star formation, the Orion Molecular Cloud, the Trapezium generation of massive stars and the other Blaauw subgroups of massive stars apparently broke out of the obscuring cloud material in few million years. A more exact analysis can be done by quantitatively considering the breakout of OB stars from their clouds, as was done in the models of Whitworth (1979) and Williams \& McKee (1997). The latter models indicate $t_{\mathrm{obsc}} \simeq 4 \mathrm{Myr}$, the number that we adopt. Because $\mathrm{B}$ stars are dominant contributors to the interstellar FUV field (see below), and because they live considerably longer than $t_{\text {obsc }}$, the exact value of $t_{\text {obsc }}$ does not significantly affect our results, i.e., most of the FUV radiation is not absorbed locally in the natal clouds of the OB stars.

In order to show the time averaged contribution of the different mass ranges to the FUV radiation field, Figures 1c and 1d show the energy output in each band for a mass distribution of stars following a Present Day Mass Function (PDMF) of MS stars. The PDMF $\varsigma_{*}(m)$ has been constructed assuming a constant star formation rate (appropriate for the solar circle-see $\S 2.4$ below), a single power-law for the IMF ( $\$ 2.1)$, and weighting the stars by their main-sequence lifetimes. Rocha-Pinto et al. (2000) have presented evidence that the Galactic star formation rate changes on time scales of order $400 \mathrm{Myr}$, which corresponds to the main-sequence lifetime of a $2.9 M_{\odot}$ star. Almost all the FUV radiation is produced by stars more massive than this, so such a time variation in the Galactic star formation rate would not affect our results. We note that the results in Figures 1c and 1d are useful only to make time-averaged estimates. To follow the evolution of the FUV-ISRF when the SFR is time and position dependent, it is necessary 
to consider that obscuration acts during the period $t_{\text {obsc }}$, starting at the moment when each generation of stars are formed.

In the FUV band the corresponding mean emissivity per unit disk area is $\sim 13.4 L_{\odot} / \mathrm{pc}^{2}$ (i.e., the area below the heavy line curve in Fig. 1c). After correcting for obscuration, the emissivity is $\sim 10.9 L_{\odot} / \mathrm{pc}^{2}$ (i.e. the area below the thin line curve in Fig. 1c). Therefore, $\sim 2.5 L_{\odot} / \mathrm{pc}^{2}$ is absorbed by the natal clouds, representing $\sim 19 \%$ of the total mean emissivity per unit disk area. The distribution of emissivity with stellar mass has a maximum at $\sim 6 M_{\odot}$ (i.e., B5), but only $\sim 16 \%$ of the total emission is produced by stars with masses below this maximum. Half of the FUV radiation is produced by stars with $m>18$, but half of the escaping FUV radiation is produced by stars with $m>13$ (i.e., earlier than B1). Stars with masses less than $3 M_{\odot}$ only contribute $\sim 3 \%$. Therefore, the SFR history in the past $\sim 350 \mathrm{Myr}$ [i.e. $t_{\mathrm{ms}}\left(3 M_{\odot}\right)$ ] is sufficient to simulate the present FUV radiation field.

The characteristics of the FUV-emitting stars can be described in terms of their mean lifetime and luminosity. Ignoring absorption by the natal cloud, we calculate the luminosity-weighted lifetime as

$$
\left\langle t_{\mathrm{FUV}}\right\rangle=\frac{\int L_{\mathrm{FUV}}(m) t_{\mathrm{ms}} d \dot{\mathcal{N}}_{*}}{\int L_{\mathrm{FUV}}(m) d \dot{\mathcal{N}}_{*}}
$$

which is $7.8 \mathrm{Myr}$ for the stellar parameters we have adopted. By comparison, the corresponding lifetime for ionizing radiation is $4.0 \mathrm{Myr}(\S 2.3)$. The mean FUV luminosity per high-mass star in the IMF is

$$
\left\langle L_{\mathrm{FUV}}\right\rangle_{h} \equiv \frac{1}{\dot{\mathcal{N}}_{* h}} \int L_{\mathrm{FUV}}(m) d \dot{\mathcal{N}}_{*}=\frac{\Gamma}{\phi_{h}} \int L_{\mathrm{FUV}}(m)\left(\frac{m_{h}}{m}\right)^{\Gamma} d \ln m .
$$

The mean FUV emissivity per unit disk area is related to the high-mass star formation rate as

$$
\Sigma_{\mathrm{FUV}}=\dot{\varsigma}_{* h}\left\langle L_{\mathrm{FUV}}\right\rangle_{h}\left\langle t_{\mathrm{FUV}}\right\rangle
$$

For the stellar parameters we have adopted $\left\langle L_{\mathrm{FUV}}\right\rangle_{h}=5.8 \times 10^{4} L_{\odot}$, and using the high-mass star formation rate derived in $\S 2.4, \Sigma_{\mathrm{FUV}}=13.4 L_{\odot} / \mathrm{pc}^{2}$ in accordance with the value derived from the PDMF above. Since $\Sigma_{\mathrm{FUV}}=10.9 L_{\odot} / \mathrm{pc}^{2}$ after correcting for obscuration, the obscuration-corrected mean luminosity is $\left\langle L_{\mathrm{FUV}}\right\rangle_{h}=4.7 \times 10^{4} L_{\odot}$.

In the $\mathrm{H}_{2}$ band, the radiation field is dominated by more massive stars. The mean emissivity per unit disk area is $\sim 3.2 L_{\odot} / \mathrm{pc}^{2}$. After correcting for obscuration, the emissivity is $\sim 2.5 L_{\odot} / \mathrm{pc}^{2}$. The maximum contribution per unit mass occurs at $m=9$ (i.e., B2 stars). After correcting for obscuration, about $85 \%$ of the total emission in the band is produced by stars with masses

\footnotetext{
${ }^{4}$ This calculation has interesting implications. If the diffuse ISM is optically thick to FUV photons, then $81 \%$ of the IR luminosity from galactic dust heated by FUV photons will arise from the diffuse gas. Therefore, when comparing IR observations of galaxies made with rather large beams, encompassing many GMCs and many FUV optical depths in the galactic plane, with PDR models (e.g., Malhotra et al. 2001, Kaufman et al. 1999) one will need to separate the IR continuum and $[\mathrm{CII}]$ and $[\mathrm{OI}]$ fine structure line emission into two components: one from the natal GMCs and one from the diffuse ISM.
} 
$m>9$, and stars with masses less than $5 M_{\odot}$ only contribute $\sim 2 \%$ to the total emission (lifetime $\sim 100 \mathrm{Myr})$. Half of the total escaping emission is produced by stars with masses over $\sim 21 M_{\odot}$ (lifetime of $\sim 9 \mathrm{Myr}$ ). Since the extinction in the $\mathrm{H}_{2}$ band is larger than the extinction in the FUV band, the radiation field in the $\mathrm{H}_{2}$ band is more dominated by nearby associations. Therefore, the amplitudes of the fluctuations in the $\mathrm{H}_{2}$ band are expected to be larger than in the FUV band, where more sources contribute to the field. For the stellar parameters we have adopted and ignoring absorption by the natal cloud, the luminosity-weighted lifetime is $\left\langle t_{\mathrm{H}_{2}}\right\rangle=6.3 \mathrm{Myr}$ and the mean $\mathrm{H}_{2}$ luminosity per high-mass star in the IMF is $\left\langle L_{\mathrm{H}_{2}}\right\rangle_{h}=1.8 \times 10^{4} L_{\odot}$. Since $\Sigma_{\mathrm{H}_{2}}=2.5 L_{\odot} / \mathrm{pc}^{2}$ after correcting for obscuration, the obscuration-corrected mean luminosity is $\left\langle L_{\mathrm{FUV}}\right\rangle_{h}=1.0 \times 10^{4} L_{\odot}$.

Most of the conclusions in the preceding two paragraphs apply only to a distribution of FUV sources that is approximately constant in both space and time. In fact, the FUV sources are located in stellar associations that are spatially and temporally very inhomogeneous. As a result, the local SFR, and therefore the FUV and $\mathrm{H}_{2}$ band emissivities, are below their mean values most of the time (see §4.3). In addition, massive stars are scarce and therefore distant, so that they are subject to relatively large interstellar extinction. All of these effects will be included in our time dependent model. In any case, the results in Figures 1c and $1 \mathrm{~d}$ show that it is sufficient to consider only the contribution of stars with masses larger than 2 or $3 M_{\odot}$ to compute the time dependence of the FUV radiation field.

\subsection{Stellar Sources of Ionizing Radiation}

Ionizing radiation from hot stars is considered in the present model because we are calculating the FUV-ISRF in the neutral gas and therefore it is necessary to verify that the sampling point is outside of HII regions. In addition, we use the observations of the total ionizing photon production rate in the Galaxy to constrain the birth rate of associations (MW97; see $\S 4$ ).

Let $s(m)$ be the time-averaged main-sequence ionizing photon luminosity (in photons $\mathrm{s}^{-1}$ ) of a star of initial mass $m$. Values of $s(m)$ were kindly provided by Vacca (private communication) and are plotted in Figure 2a as $s_{49}=s /\left(10^{49}\right.$ photons $\left.\mathrm{s}^{-1}\right)$. An analytic fit to these data is given in Table 1. In Vacca, Garmany, \& Shull (1996) the ionizing luminosities are given as function of the spectroscopic mass and the evolutionary mass, but here the zero age main sequence mass is used because the IMF $(\S 2.1)$ refers to this initial mass.

The contribution of the different mass ranges to the ionizing photon production is shown in Figure 2b. Again a mass distribution of stars following a PDMF was assumed. For the adopted distribution (constant SFR and an IMF with power law $\Gamma=1.35$, see $\S 2.1$ ), stars of about $30 M_{\odot}$ make the maximum contribution. Half of the ionizing photons are produced by stars above $\sim 55 M_{\odot}$ (lifetime $\left.\sim 3.8 \mathrm{Myr}\right)$, and $\sim 95 \%$ are produced by stars above $\sim 20 M_{\odot}$.

The characteristics of the ionizing luminosity from a group of stars following an IMF that is a 
cutoff power law can be described by two quantities (McKee 1989). The first is the mean ionizing photon luminosity per high-mass star,

$$
\langle s\rangle_{h} \equiv \frac{1}{\dot{\mathcal{N}}_{* h}} \int s(m) d \dot{\mathcal{N}}_{*}=\frac{\Gamma}{\phi_{h}} \int s(m)\left(\frac{m_{h}}{m}\right)^{\Gamma} d \ln m,
$$

where the second equation follows from equation (5). The second quantity is the mean lifetime of an ionizing star,

$$
\left\langle t_{\mathrm{ion}}\right\rangle=\frac{\int s(m) t_{\mathrm{ms}} d \dot{\mathcal{N}}_{*}}{\int s(m) d \dot{\mathcal{N}}_{*}}
$$

If each star produces $Q(m)=s(m) t_{\mathrm{ms}}$ ionizing photons during its lifetime, then these equations imply that in a steady state the expected value of the ionizing luminosity from a group of stars is

$$
S=\int Q(m) d \dot{\mathcal{N}}_{*}=\dot{\mathcal{N}}_{* h}\langle s\rangle_{h}\left\langle t_{\text {ion }}\right\rangle
$$

For the stellar parameters we have adopted, $\langle s\rangle_{h, 49}=0.42$ and $\left\langle t_{\text {ion }}\right\rangle=4.0 \times 10^{6}$ yr, so that

$$
\dot{\mathcal{N}}_{* h}=6.0 \times 10^{-7} \phi_{S} S_{49}
$$

where $\dot{\mathcal{N}}_{* h}$ is measured in $\mathrm{yr}^{-1}$ and $\phi_{S}$ allows for deviations from our adopted values of $\langle s\rangle_{h}$ and $\left\langle t_{\text {ion }}\right\rangle$. If $m_{u}$ differs from our adopted value of $120, \phi_{S}$ changes. For example, $\phi_{S}=1.32$ for $m_{u}=80$ and $\phi_{S}=0.88$ for $m_{u}=150$; a power-law approximation over this range gives $\phi_{S} \simeq\left(120 / m_{u}\right)^{0.65}$. By comparison MW97, who adopted $\Gamma=1.5$ and used a different set of stellar lifetimes but the same value of $m_{u}$, found $\langle s\rangle_{h, 49}=0.34$ and $\left\langle t_{\text {ion }}\right\rangle=3.7 \times 10^{6} \mathrm{yr}$, corresponding to $\phi_{S}=1.32$.

\subsection{Star Formation in the Galaxy}

What is the relation between the star formation rate locally and that in the Galaxy as a whole? Let $\dot{\zeta}_{* h}(R)$ be the birthrate of massive stars $\left(m>m_{h}\right)$ per unit area at Galactocentric radius $R$. MW97 found that the spatial distribution of massive star formation in the Galaxy (omitting the Galactic Center) can be approximated as $\dot{\zeta}_{* h}(R) \propto \exp \left(-R / H_{R}\right)$ for $11 \mathrm{kpc}>R>$ $3 \mathrm{kpc}$, with $H_{R}=3.5 \mathrm{kpc}$. The total rate of massive star formation in the Galaxy is then

$$
\begin{aligned}
\dot{\mathcal{N}}_{* h, T} & =\dot{\varsigma}_{* h} \int_{3 \mathrm{kpc}}^{11 \mathrm{kpc}} 2 \pi \exp \left[\left(R_{0}-R\right) / H_{R}\right] R^{2} d R \\
& \equiv \dot{\varsigma}_{* h} A_{\mathrm{eff}},
\end{aligned}
$$

where $R_{0}=8.5 \mathrm{kpc}$ is the distance to the Galactic Center and $\dot{\zeta}_{* h}$ without an argument is the value of the massive star formation rate at the solar circle. [This definition of $A_{\text {eff }}$ differs by a factor $\exp \left(R_{0} / H_{R}\right)$ from that in MW97]. Numerically, the effective area for massive star formation in the Galaxy is $A_{\text {eff }}=530 \mathrm{kpc}^{2}$. It should be borne in mind that star formation is observed to 
occur beyond $11 \mathrm{kpc}$, but there are no giant radio HII regions, and therefore few if any large associations, there.

Radio surveys of the Galaxy provide an estimate for the total ionizing photon production rate, $S_{T}=2.6 \times 10^{53}$ photons s $^{-1}$, good to a factor of about 1.5 (MW97). We use $S_{T}$ to set $\dot{\zeta}_{* h}$, since equations (13) and (15) yield

$$
\dot{\varsigma}_{* h}=6.0 \times 10^{-7} \phi_{S}\left(\frac{S_{T, 49}}{A_{\text {eff }}}\right) \rightarrow 29.4 \phi_{S} \quad \mathrm{kpc}^{-2} \mathrm{Myr}^{-1}
$$

where we have expressed the result in convenient units. This result is compared with other estimates in the literature in Figure 3 . We note that the value $\dot{\zeta}_{* h}=38 \mathrm{kpc}^{-2} \mathrm{Myr}^{-1}$ derived in MW97 was obtained by assuming $\Gamma=1.5$ and adopting a different set of stellar lifetimes.

In the Galaxy as a whole, the birthrate of massive stars is $6.0 \times 10^{-7} \phi_{S} S_{49, T}=0.0156 \phi_{S} \mathrm{yr}^{-1}$. Correspondingly, the time between core-collapse supernovae in the Galaxy is $t_{S N} \sim\left(64 / \phi_{S}\right) \mathrm{yr}$.

The resulting PDMF for $m>m_{h}$ agrees reasonably well with the one derived by MS79 from observed data. Our PDMF is systematically higher than the one derived by Scalo (1986), although it is close to the top of his error bars. This trend can be explained by the fact that our PDMF corresponds to the mean star formation rate at the solar circle as determined from the Galactic distribution of emission of ionizing photons, whereas the observed local PDMF likely corresponds to the median star formation rate (see $\S 4.3$ ). Also, massive stars embedded or close to molecular clouds might be missing in the observed PDMF.

\section{Associations}

The sources of FUV radiation are the short-lived massive stars that generally form in associations. Therefore, these associations can be considered as point sources with an FUV luminosity that decreases with time as their massive members die. These associations tend to expand (typical velocities are $\sim 1-2 \mathrm{~km} \mathrm{~s}^{-1}$ ), but this effect can be neglected because the associations remain small during the time that most of the FUV radiation is produced - recall that a large fraction of the FUV radiation that escapes from the natal cloud escapes during the first $16 \mathrm{Myr}(\S 2.2)$. The fact that the main contributors to the FUV field are grouped has a tremendous effect on the temporal behavior of the radiation field. For example, the occurrence of a close association can raise the local radiation field by orders of magnitude until its massive

star population dies. Therefore, we must model the birth-rate and size-distribution of associations in order to determine the time and space dependence of the interstellar FUV radiation field. We adopt the MW97 model, which explicitly gives the birthrate of associations as a function of the number of stars formed in the association. The birth-rate distribution in MW97 is derived from observations of HII regions, and reproduces the total ionizing luminosity, the local ionizing luminosity, and the rate of core collapse supernovae observed in the Galaxy. Moreover, the observed OB associations in the solar neighborhood (i.e., those that are young and large enough 
to be identified) are consistent with the MW97 model, as we discuss below. The MW97 model is based on observations of the effects produced by Lyman continuum photons radiated by a small fraction of the stellar population (i.e. the very hot stars). A larger fraction of the star population contributes to the FUV radiation field; therefore an additional test to the MW97 model will be provided here.

\subsection{Evolution of the Luminosity of Associations}

The typical period of time that an association forms stars is of the order of $15-20 \mathrm{Myr}$ (Blaauw 1964, 1991; Heiles 1990). The rate at which stars form in an association is not continuous but occurs in bursts during the 15-20 Myr interval. We idealize this situation by assuming that each association produces five bursts, or generations, each of which has the same number of stars with a stellar mass distribution given by equation (3). We characterize the size of the association by $\mathcal{N}_{* h}$, the number of massive stars that form over the 5 generations. The stars in each generation are assumed to form simultaneously. As in MW97, we assume that these five generations occur with a period of $t_{g}=3.7 \mathrm{Myr}$. At any given time after the birth of an association, the luminosities $\mathcal{L}_{\mathrm{FUV}}$ and $\mathcal{L}_{\mathrm{H}_{2}}$ of the entire association are the sum of the luminosities of the stars still living. However, as discussed in $\S 2.2$, not all of this UV luminosity can escape the parent cloud of the association. We estimated in $\S 2.2$ that for each generation the fraction of the radiation that escapes, $f_{\text {esc }}$, goes linearly from $1 / 4$ to one in a time $t_{\text {obsc }} \sim 4 \mathrm{Myr}$. In this case, the effective luminosity of an association of age $t$ that will eventually form a total of $\mathcal{N}_{* h}$ massive stars is

$$
\mathcal{L}_{\mathrm{FUV}, \mathrm{H}_{2}}\left(\mathcal{N}_{* h}, t\right)=\sum_{j=1}^{\mathcal{N}_{g}}\left\{f_{\mathrm{esc}}\left[t-(j-1) t_{g}\right] \sum_{i \geq i_{\text {live }}(j, t)} L_{\mathrm{FUV}, \mathrm{H}_{2}}\left(m_{i}\right)\right\} .
$$

Here the first summation is over the $\mathcal{N}_{g} \leq 5$ generations or bursts that occurred during the association age $t$ [i.e., those for which $t-(j-1) t_{g}>0$ ]. The second summation is over all the stars born in generation $j$ and that are still on the main sequence at time $t$. (The stars in each generation are labeled in order of decreasing mass, and $i_{\text {live }}(j, t)$ labels the most massive star from generation $j$ still on the main sequence at time $t$.) In practice, the second summation can be stopped at $m<2 M_{\odot}$ due to the negligible FUV luminosities of such stars.

Figure 4 shows the evolution of the FUV luminosity $\mathcal{L}_{\mathrm{FUV}}\left(\mathcal{N}_{* h}, t\right)$ for associations characterized by generations with $\mathcal{N}_{* h} / 5=200,20$, and 2 high-mass stars (i.e., the associations ultimately form 1000, 100, and 10 high-mass stars, respectively). The decreases in $\mathcal{L}_{\mathrm{FUV}}$ are caused by the deaths of the most massive stars born in the early generations. The first generation is born at age $t=0$. The initial increase is due to the gradual dispersion of the dust from the natal cloud around the first generation. The final decrease is due to the death of OB stars after the last generation has formed. The right vertical axis gives the contribution to the energy density $U_{\mathrm{FUV}}$ at a point located $100 \mathrm{pc}$ away assuming that there is no intervening extinction [i.e., $\left.U_{\mathrm{FUV}}=\left(1 / 4 \pi r^{2} c\right) \mathcal{L}_{\mathrm{FUV}} /(2070 \AA-912 \AA)\right]$. Figure 4 shows that the maximum luminosity of an 
association is attained at about the time the fifth generation dissipates the absorbing material around it (i.e., about $18 \mathrm{Myr}$ after the first generation begins to form). At this time, the mean FUV luminosity per high-mass star formed (in the five generations) is $L_{\max , h} \sim 1.6 \times 10^{4} L_{\odot}$. Note that $L_{\max , h}$ is only about $28 \%$ of the value $\left\langle L_{\mathrm{FUV}}\right\rangle_{h}$ calculated in $\S 2.2$ since many of the most luminous stars in the first four generations have died by this point. The contributions from generations 1 to 5 to $L_{\max , h}$ are respectively $5.4 \%, 7.9 \%, 12.4 \% 21.6 \%$ and $52.7 \%$. At $20 \mathrm{Myr}$, the mean FUV luminosity per high-mass star formed drops to $\sim 1.0 \times 10^{4} L_{\odot}$.

\subsection{Size Distribution of Associations}

Using compilations of radio HII regions in the Galaxy and optical studies of HII regions in nearby galaxies, MW97 showed that the luminosity distribution of giant radio HII regions in the Galaxy can be fit by a truncated power law of the form $\mathcal{N}_{a}(>S)=\mathcal{N}_{a u}\left[\left(S_{u} / S\right)-1\right]$, where $S$ is the initial ionizing photon luminosity, $\mathcal{N}_{a}(>S)$ is the number of associations with luminosity at least $S, S_{u}$ is the upper limit of the distribution, and $\mathcal{N}_{a u}$ is the number of the most luminous associations, with luminosities between $0.5 S_{u}$ and $S_{u}$.

The luminosity distribution of $\mathrm{OB}$ associations does not continue as a power law in $S$ to low luminosities because when the luminosity is dominated by a single star, the distribution is determined by the IMF. MW97 assumed that the underlying dependence on the number of high-mass stars in the association did not change at this point, however, so that $\mathcal{N}_{a}\left(>\mathcal{N}_{* h}\right)=\mathcal{N}_{a u}\left[\left(\mathcal{N}_{* h, u} / \mathcal{N}_{* h}\right)-1\right]$. Here $\mathcal{N}_{* h}$ is the number of high-mass stars in all the generations of the association, and $\mathcal{N}_{* h, u}$ is the number of high-mass stars in an association in which each generation has an ionizing luminosity $S_{u}$ (MW97 argued that the different generations of an association had comparable ionizing luminosities). If the lifetime of the association is $t_{a}$, the birthrate of associations in which at least $\mathcal{N}_{* h}$ high-mass stars form is then

$$
\dot{\mathcal{N}}_{a}\left(>\mathcal{N}_{* h}\right)=\frac{\mathcal{N}_{a u}}{t_{a}}\left(\frac{\mathcal{N}_{* h, u}}{\mathcal{N}_{* h}}-1\right)
$$

MW97 assumed that associations had 5 generations, each lasting $3.7 \mathrm{Myr}$, so that $t_{a}=18.5$ Myr; we shall adopt this value here. For the Galaxy, MW97 estimate an upper bound $S_{u}=490 \times 10^{49}$ photons s $^{-1}$, which corresponds to $S_{u} /\langle s\rangle_{h}=1180$ massive stars per generation, or $\mathcal{N}_{* h, u}=5900$ massive stars over the life of the association. This is somewhat smaller than the value $\mathcal{N}_{* h, u}=7200$ estimated by MW97 because of the flatter IMF we have adopted here. The lower limit of the distribution is $\mathcal{N}_{* h, l}=\mathcal{N}_{* h, u} \exp \left[-S_{T} /\left(\mathcal{N}_{a u} S_{u}\right)\right]$, which is obtained by requiring that the integrated ionizing photon emission by the entire distribution of associations match the observed value. With $S_{T}=2.6 \times 10^{53}$ photons s$^{-1}$ and $\mathcal{N}_{a u}=6.1$ (MW97), we find $\mathcal{N}_{* h, l} \simeq 1.0$.

The birthrate of associations per unit disk area is $\dot{\zeta}_{a}\left(>\mathcal{N}_{* h}\right)=\dot{\mathcal{N}}_{a}\left(>\mathcal{N}_{* h}\right) / A_{\text {eff }}$. At the solar circle the effective area is $A_{\text {eff }} \sim 530 \mathrm{kpc}^{2}$, so that the birthrate of associations per unit disk area is

$$
\dot{\varsigma}_{a}\left(>\mathcal{N}_{* h}\right)=6.2 \times 10^{-4}\left(\frac{5900}{\mathcal{N}_{* h}}-1\right) \quad \mathrm{kpc}^{-2} \mathrm{Myr}^{-1} .
$$




\subsection{The Typical Star Formation Rate is Below Average}

Equation (19) reveals that, on average, one association (of any size, $\mathcal{N}_{* h, l}<\mathcal{N}_{* h}<\mathcal{N}_{* h, u}$ ) forms in one $\mathrm{kpc}^{2}$ each $\sim 0.3 \mathrm{Myr}$ at the solar circle. The number of massive stars formed in a given area $A$ and over a given time interval $\Delta t$ will depend on the size $\mathcal{N}_{* h}$ and number $\mathcal{N}_{a}$ of associations formed. For small areas (e.g., $\sim 1 \mathrm{kpc}^{2}$ ) large fluctuations are expected even if $\Delta t$ is tens of millions of years. We shall show that as a result, the typical rate of massive star formation $\dot{\zeta}_{* h \text {,typ }}$ can be significantly less than the average rate in the Galaxy for small values of $A \Delta t /\left(A_{\text {eff }} t_{a}\right)$. Since the FUV-ISRF is determined by the average rate of massive star formation in a small fraction of the disk over a relatively short time, this means that the typical value of the radiation field is significantly less than the average value.

The number of associations $\mathcal{N}_{a}\left(>\mathcal{N}_{* h}\right)$ with at least $\mathcal{N}_{* h}$ high-mass stars that are expected to form during a period of time $\Delta t$ inside an area $A$ is

$$
\mathcal{N}_{a}\left(>\mathcal{N}_{* h}, \Delta t, A\right)=\frac{\dot{\mathcal{N}}_{a}\left(>\mathcal{N}_{* h}\right) A \Delta t}{A_{\text {eff }}}
$$

Conversely, the largest $\mathcal{N}_{a}$ associations formed in an area $A$ and time $\Delta t$, can be expected to have at least

$$
\mathcal{N}_{* h}\left(\mathcal{N}_{a}\right)=\frac{\mathcal{N}_{* h, u}}{1+\left(\frac{A_{\text {eff }} t_{a}}{\mathcal{N}_{a u} A \Delta t}\right) \mathcal{N}_{a}}
$$

high-mass stars, where we have used equation $(18)$ to evaluate $\dot{\mathcal{N}}_{a}$. For example, consider a large sample of sets of associations formed in $A \Delta t$; the median size of the largest association in each set

will be $\mathcal{N}_{* h}\left(\mathcal{N}_{a}=\frac{1}{2}\right)$ (i.e., it is expected that half of the sets of associations formed would have an association with $\mathcal{N}_{* h}$ larger than this value). As a numerical example, $A=1 \mathrm{kpc}^{2}$ and $\Delta t=25$ Myr, give $\mathcal{N}_{* h}\left(\mathcal{N}_{a}=\frac{1}{2}\right)=178$, which is substantially less than $\mathcal{N}_{* h, u}=5900$.

This analysis carries over into comparing the mean and median rates of star formation in a given area of the Galaxy. The important point is that, averaged over a finite region of space and time, the star formation rate is most likely a relatively low value, but that if one waits long enough or chooses a large enough area, a very massive association will form in this region that raises the mean rate above the median or typical rate. For an area $A$ and time interval $\Delta t$, the typical star formation rate can be estimated as the rate of formation in all but the largest association plus the rate of formation in the typical largest association,

$$
\dot{\zeta}_{* h} \simeq \frac{1}{A_{\text {eff }}} \int_{\mathcal{N}_{* h, l}}^{\mathcal{N}_{* h}(1)} \mathcal{N}_{* h} d \dot{\mathcal{N}}_{a}+\frac{1}{A_{\text {eff }}} \mathcal{N}_{* h}\left(\frac{1}{2}\right) \dot{\mathcal{N}}_{a}\left[\mathcal{N}_{* h}(1)\right]
$$

The ratio of this typical rate to the mean rate can be evaluated with the aid of equation (18),

$$
\frac{\dot{\zeta}_{* h, \text { typ }}}{\left\langle\dot{\zeta}_{* h}\right\rangle} \simeq \frac{\ln \left[\frac{\mathcal{N}_{* h}(1)}{\mathcal{N}_{* h, l}}\right]+2\left[1-\frac{\mathcal{N}_{* h}\left(\frac{1}{2}\right)}{\mathcal{N}_{* h, u}}\right]}{\ln \left(\frac{\mathcal{N}_{* h, u}}{\mathcal{N}_{* h, l}}\right)} .
$$


For example, if the typical star formation rate is measured by observing the $\mathrm{O}$ and $\mathrm{B}$ stars, then we are only probing a time $\Delta t \sim 25 \mathrm{Myr}$, and the typical to mean ratio within $1 \mathrm{kpc}$ is $\sim 0.86$. We show in $\S 4$ that most of the typical FUV-ISRF originates from associations closer than $\sim 400$ $\mathrm{pc}$ and younger than $\sim 30 \mathrm{Myr}$; in this case, the ratio of typical to mean is 0.69 . Moreover, when averaging over very long periods of time, about half of the local mean FUV-ISRF is produced by very infrequent large associations within less than $H_{*} \sim 85 \mathrm{pc}$, the observed scale height of $\mathrm{OB}$ stars. The ratio of the typical to mean star formation within $H_{*}$ during a period of $30 \mathrm{Myr}$ is $\sim 0.34$. In other words, for very long periods of time there are no large associations within $H_{*}$ and the FUV field is relatively low. Then, sporadically, a large association forms within $H_{*}$ and raises the field so much that, even though its duration is brief, it pushes the mean significantly above the median. Properly accounting for this effect, the typical FUV-ISRF is about half the mean value!

As a sidenote to this section, it is interesting to compare the predictions of the MW97 model with observations of nearby OB associations. In total, about 7 associations younger than $25 \mathrm{Myr}$ are observed within $1 \mathrm{kpc}$ from the Sun. Equation (20) predicts that the 7 associations will each produce more than $\sim 40$ high-mass stars. The completeness of the observed sample is unknown, but the observations appear consistent with the model. MW97 came to the same conclusion by a slightly different approach: They calculated that there should be 7.3 associations within a kpc of the Sun that currently have at least 30 high-mass stars (i.e., excluding some young associations that have yet to reach their full complement of high-mass stars) and are younger than 25 Myr. In summary, the MW97 model for the OB associations is in good agreement with

the observed associations in the Solar neighborhood. However, the observational bias is such that these associations are large and young. A significant contribution to the FUV-ISRF comes from smaller and/or older associations that are less readily detected. We shall use the MW97 model to describe these associations as well.

\section{Time dependent FUV-ISRF}

To calculate the evolution of the FUV-ISRF at a given point in the Galaxy, we assume that the birthrate of associations per unit volume has a Gaussian distribution with respect to the height $z$ above the galactic plane. We take the scale height of the birthrate to be $H_{*}=85 \mathrm{pc}$, the observed scale height of molecular gas and OB stars (Ferrière 1998, and references therein). Since the surface number density of young associations is small, the above distributions must be regarded as probabilistic. In the plane the distribution is assumed to be uniform (random) since almost all the FUV-ISRF originates within $\sim 1 \mathrm{kpc}$, the scale length for dust attenuation of FUV, and therefore radial gradients in the birthrates are negligible. Large scale patterns of star formation, such as spiral arms, are present in the Galaxy, but it is not necessary to include the corresponding spatial variation in the star formation rate since the FUV field at a given point is mainly determined by the population of massive stars in a rather small region around it (within $0.4 \mathrm{kpc}$ ). Our adopted star formation rate is based on the global model of MW97 and is therefore 
an average over arm and interarm regions of the Galaxy. Since the fluctuations in the ISRF we calculate are purely statistical, they are a lower limit to the fluctuations that occur as a point moves between arm and interarm regions.

Figure 5 shows the projection on the plane of the positions of associations formed in a period of $500 \mathrm{Myr}$ (left panel) and $30 \mathrm{Myr}$ (right panel). The birthrate corresponds to the average value at the solar circle. Note that although there are more small associations than large associations, there are equal total numbers of stars in small and large associations. For a point at the center, most of the FUV radiation comes from within the circle. The FUV flux varies as function of position (or time), depending on whether the given point is close to large associations.

The distribution of attenuating dust with respect to $z$ is assumed to follow the distribution of HI as given by Dickey \& Lockman (1990), i.e.,

$$
n_{\mathrm{H}}(z)=0.566\left[0.69 e^{-(z / 127 \mathrm{pc})^{2}}+0.189 e^{-(z / 318 \mathrm{pc})^{2}}+0.113 e^{-|z| / 403 \mathrm{pc}}\right] \mathrm{cm}^{-3} .
$$

Note that $n_{\mathrm{H}}$ is the space-averaged number of $\mathrm{H}$ atoms in a large volume that might contain a significant amount of hot gas (McKee \& Ostriker 1977). The molecular gas is not considered due to its small filling factor. The dust optical depth to a given association is then assumed to be proportional to the column density of $\mathrm{H}$ atoms, $N(\mathrm{HI})$, along the line of sight. We adopt the physical properties of dust proposed by Witt and Gordon (1999) to estimate the average absorption and scattering by dust grains in the 912 - $1100 \AA$ Aband and in the $1100-2070$ Aband. The scattering and absorption optical depths are given by $\tau_{s, a}=\kappa_{s, a} N(\mathrm{HI})$, where the values of $\kappa_{s, a}$ are given in Table B1. The assumption of a constant extinction per unit column density is justified in statistical studies of the expected radiation field, but this approximation is not useful in calculating the contribution of a particular source because the extinction per unit column density is known to depend on the particular line of sight. Scattering removes photons from the direct line of sight to the associations, but adds photons from other directions. The effect of scattering is considered in Appendix B.

The contribution of an association labeled " $i$ " to the radiation density at a distance $r$ can be approximated as

$$
u_{\text {band }}(i)=\frac{1}{4 \pi r^{2} c} \frac{\mathcal{L}_{\text {band }}(i)}{\Delta_{\text {band }}} e^{-\tau_{\text {band }}(i)},
$$

where $c$ is the light speed, and $\mathcal{L}_{\text {band }}(i)$ is the effective luminosity (eq. 17) of the association labeled $i$, which has $\mathcal{N}_{* h}$ high-mass stars and whose age is $t$. The term $e^{-\tau_{\text {band }}}$ accounts for both absorption and scattering (see Appendix B).

At a given time $t_{\text {sim }}$ of the simulation, the total energy density in each band $U_{\text {band }}\left(t_{\text {sim }}\right)$ is the sum of the contributions $u_{\text {band }}(i)$ from all the associations formed between $t=0$ and $t=t_{\text {sim. Due }}$ to the $1 / r^{2}$ term in equation (25), the resulting radiation density $U_{\text {band }}$ at a given point is very sensitive to the spatial distribution of the associations; in particular, a nearby luminous source can dominate the radiation field. The effect of extinction is very important for distant sources. Without extinction the contribution of FUV sources in a disk grows logarithmically with distance; 
with extinction the total FUV field comes from sources within a few optical depths. In practice it is sufficient to consider only associations within about $1 \mathrm{kpc}$ for conditions at the solar circle of the Galaxy. Additionally, old associations (with ages exceeding the lifetime of stars with masses $\left.\sim 2-3 M_{\odot}\right)$ make a negligible contribution to $U_{\mathrm{FUV}}$. These two facts are useful in reducing the number of associations that must be tracked in simulations to obtain an accurate accounting of the time dependence of the radiation field at a given point.

\subsection{The Mean FUV-ISRF}

Before presenting the results from simulations, it is useful to estimate the mean FUV energy density assuming a smooth emissivity per unit volume corresponding to the PDMF and a smooth spatial distribution; hereafter the "continuous method". This approach is necessary because the "discrete method" used in the simulation (assuming that FUV sources are highly discrete in space and time) is useful to get the median value of the radiation field, but is very inefficient in calculating the mean value because of the large integration times needed to obtain a good sample of rare events. However, the "continuous method" does not show the interesting time dependence of the FUV-ISRF. Knowledge of the ratio between the median and the mean value of the radiation field is important because low resolution observations (for example, in external galaxies) that average over huge spatial volumes can provide an estimate of the mean FUV-ISRF, but the interstellar gas is subject typically to the median FUV-ISRF.

The mean energy density $<U_{\text {band }}>$ in the plane can be calculated by adding the diluted extincted and scattered radiation from a smooth distribution of stars following a PDMF. Let

$$
n_{*}(m, \mathbf{r}) \equiv \frac{d^{2} \mathcal{N}_{*}(m, \mathbf{r})}{d V d \ln m}
$$

be the PDMF per unit volume at a point $\mathbf{r}$. The mean energy density of radiation at the origin is then

$$
\left\langle U_{\text {band }}\right\rangle=\frac{1}{c \Delta_{\text {band }}} \int \frac{e^{-\tau_{\text {band }}(\mathbf{r})}}{4 \pi r^{2}} d V \int_{m_{l}}^{m_{u}} n_{*}(m, \mathbf{r}) L_{\text {band }}(m) d \ln m,
$$

where $L_{\text {band }}(m)$ is the mean luminosity of a star of mass $m$ after subtracting the fraction lost in the parent cloud. As shown in Appendix B, we approximate the optical depth, including both absorption and scattering, as $\tau_{\text {band }}=\tau_{a}+\tau_{s}(1-\mathcal{R})$, where $\mathcal{R}$ is a factor that is somewhat less than unity for scattering that is strongly peaked in the forward direction.

Since the stars that dominate the FUV flux are relatively nearby, we can ignore the Galactic radial gradient in the density of stars and express the volume density of stars in terms of the surface density as

$$
n_{*}(m, \mathbf{r})=\varsigma_{*}(m) D(z)
$$


where $z$ is the distance normal to the plane and, for a Gaussian distribution,

$$
D(z)=\frac{e^{-\left(z / H_{*}\right)^{2}}}{\sqrt{\pi} H_{*}} .
$$

Recall that for a constant birthrate, the PDMF for stars younger than the age of the disk is related to the star formation rate by $\varsigma_{*}(m)=\dot{\varsigma}_{*}(m) t_{\mathrm{ms}}(m)$. Equation (27) can then be rewritten as

$$
\left\langle U_{\text {band }}\right\rangle=\frac{4 \pi \mathcal{J}_{\text {band }} \Sigma_{L_{\text {band }}}}{c \Delta_{\text {band }}},
$$

where

$$
\Sigma_{L_{\mathrm{band}}}=\int_{m_{l}}^{m_{u}} \varsigma_{*}(m) L_{\mathrm{band}}(m) d \ln m
$$

is the mean rate of emission of radiation (after subtracting the absorbed emission in the natal clouds) per unit disk area in the band, and

$$
4 \pi \mathcal{J}_{\text {band }}=\int \frac{e^{-\tau_{\text {band }}(\mathbf{r})} D(z)}{4 \pi r^{2}} d V
$$

is $4 \pi$ times the mean intensity due to unit FUV emissivity in the disk.

At the solar circle $\Sigma_{L_{\mathrm{H} 2}} \sim 2.5 L_{\odot} / \mathrm{pc}^{2}$ and $\Sigma_{L_{\mathrm{FUV}}} \sim 10.9 L_{\odot} / \mathrm{pc}^{2}$. Numerical integration of equation (32), taking into account multiple scattering (Appendix B), gives respectively 0.88 and 1.19 for the value of $4 \pi \mathcal{J}_{\text {band }}$ in the $\mathrm{H}_{2}$ and $\mathrm{FUV}-\mathrm{H}_{2}$ bands. From equation (30) one obtains $\left\langle U_{\mathrm{H} 2}\right\rangle=15.7 \times 10^{-17} \mathrm{erg} \mathrm{cm}^{-3} \AA^{-1},\left\langle U_{\mathrm{FUV}-\mathrm{H} 2}\right\rangle=13.8 \times 10^{-17} \mathrm{erg} \mathrm{cm}^{-3} \AA^{-1}$, and $\left\langle U_{\mathrm{FUV}}\right\rangle=14.2 \times 10^{-17} \mathrm{erg} \mathrm{cm}^{-3} \AA^{-1}$. Additionally, $\left\langle U_{1400 \AA}\right\rangle=14.4 \times 10^{-17} \mathrm{erg} \mathrm{cm}^{-3} \AA^{-1}$. Hereafter, we denote these mean values as $\left\langle U_{\text {band }}\right\rangle_{\infty}$ since they correspond to the mean energy density for an infinite period of time.

To clarify how different regions contribute to the mean radiation field, consider a simple model consisting of two regions: the first is a sphere of radius $H_{*}$ centered at a point in the midplane, and the second is a zero thickness disk that covers the midplane but excludes a radius $H_{*}$ around the point in question. The volume number density per unit mass of FUV sources in the sphere is $n_{*}(m)=\varsigma_{*}(m) / 2 H_{*}$, and the surface density in the disk is $\varsigma_{*}(m)$. The UV extinction is assumed to be $e^{-k r}$, where $k=\left[\kappa_{a}+\kappa_{s}(1-\mathcal{R})\right] n_{\mathrm{H}} \simeq 7 \times 10^{-22} \mathrm{~cm}^{-1}$ (see Appendix B). Then,

$$
\left\langle U_{\mathrm{band}}\right\rangle \simeq \frac{1}{c \Delta_{\text {band }}}\left[\frac{1}{2 H_{*}} \int_{0}^{H_{*}} e^{-k r} d r+\frac{1}{2} \int_{H_{*}}^{\infty} \frac{e^{-k r}}{r} d r\right] \int_{m_{l}}^{m_{u}} \varsigma_{*}(m) L_{\mathrm{band}}(m) d \ln m .
$$

The two terms in the bracket correspond to $4 \pi \mathcal{J}$ in this approximation. Since $k H_{*} \ll 1$, the contribution of the sources in the sphere to the function $4 \pi \mathcal{J}$ is $\sim 1 / 2$. This term represents sources out to a distance of order the scale height $H_{*}$ of the disk. Beyond $H_{*}$, the contribution of the sources in the disk is $\frac{1}{2} E_{1}\left(k H_{*}\right)$, where $E_{1}$ is the first exponential integral. Since $k H_{*} \simeq 0.18$ and $E_{1}(0.18) \simeq 1.3$, the second term is about 0.65. In other words, in calculating the mean field, we find that roughly half the radiation comes from sources closer than $\sim H_{*}=85 \mathrm{pc}$, and half the 
radiation arises from more distant sources in the disk. However, as shown in equation (23), the typical number of FUV sources inside of $85 \mathrm{pc}$ is much less than the mean value. Therefore, most of the time, the FUV radiation field is expected to be well below its mean value. We also see that beyond $r>k^{-1} \sim 0.46 \mathrm{kpc}$ the contribution to the FUV field rapidly falls off due to attenuation of the distant FUV flux by dust.

In order to quantify the simulation time necessary to get a good estimate of $\langle U\rangle$, we calculate the accumulated typical FUV radiation field during a period of time $t_{\text {sim }}$,

$$
\left\langle U_{\text {band }}\right\rangle_{t_{\text {sim }}}=\frac{1}{c \Delta_{\text {band }}} \int \frac{e^{-\tau_{\text {band }}(\mathbf{r})} D(z)}{4 \pi r^{2}} d V \int \varsigma_{*, \text { typ }}\left(m, A=\pi r^{2}, t_{\text {sim }}\right) L_{\text {band }}(m) d \ln m .
$$

Here the typical PDMF is

$$
\begin{aligned}
\varsigma_{* \text { typ }}\left(m, A, t_{\mathrm{sim}}\right) & =\int_{0}^{\min \left(t_{\mathrm{sim}}, t_{\mathrm{ms}}\right)} \dot{\varsigma}_{* \mathrm{typ}}\left(m, A, t_{\mathrm{sim}}\right) d t \\
& =\left\{\frac{\ln \left[\frac{\mathcal{N}_{* h}(1)}{\left.\mathcal{N}_{* h, l}\right]+2\left[1-\frac{\mathcal{N}_{* h}\left(\frac{1}{2}\right)}{\mathcal{N}_{* h, u}}\right]}\right.}{\ln \left(\frac{\mathcal{N}_{* h, u}}{\mathcal{N}_{* h, l}}\right)}\right\} \dot{\zeta}_{*}(m) \min \left(t_{\mathrm{sim}}, t_{\mathrm{ms}}\right),
\end{aligned}
$$

where the typical maximum value of $\mathcal{N}_{* h}$ in the area $A$ during time interval $\Delta t=t_{\text {sim }}$ is given by equation (21). This equation applies only if $A t_{\text {sim }}$ is large enough that $\mathcal{N}_{* h \text {,typ max }}>\mathcal{N}_{* h, l}$; for smaller values of $A t_{\text {sim }}, \varsigma_{* \operatorname{typ}}\left(m, A, t_{\text {sim }}\right)=0$.

Note that in computing $\left\langle U_{\text {band }}\right\rangle_{t_{\text {sim }}}$ for a given spatial point, the area of simulation $A_{\text {sim }}$ must be chosen so that $\tau_{\text {band }} \gg 1$ at the outer boundary $r_{\max }$ (see eq. (34)). Otherwise, the simulation area would not include significant contributors to the UV flux at the central point. This is equivalent to $r_{\max } \gg k^{-1}$, which for our standard values of $n_{\mathrm{H}}$ and $\kappa_{a, s}$ results in $r_{\max } \gg 0.46 \mathrm{kpc}$. Assuming a square simulation area, this corresponds to $A_{\text {sim }} \gg 0.85 \mathrm{kpc}^{2}$. Given that $A_{\text {sim }} \simeq 5$ $\mathrm{kpc}^{2}$, the simulation area is no longer a factor in $\langle U\rangle$; only the simulation time enters. Figure 6 shows the ratio $\left\langle U_{\text {band }}\right\rangle_{t_{\text {sim }}} /\left\langle U_{\text {band }}\right\rangle_{\infty}$ as function of the time $t_{\text {sim }}$. Extremely long times are required to get a good estimate of $\left\langle U_{\text {band }}\right\rangle$ from the simulations.

\subsection{Simulation in the Galactic Plane at the Solar Circle}

Over a sufficiently large area $A_{\text {sim }}$ to include all possible contributions to the FUV field not severely attenuated by dust, the associations form following the truncated birthrate law given by equation (19). We assume a constant interval of time $\Delta t_{a \text {, form }}$ between the formation of associations in the simulation area $A_{\text {sim. }}$. Equation (19) gives this interval as

$$
\Delta t_{a, \text { form }}=\frac{A_{\mathrm{eff}} t_{a}}{\mathcal{N}_{a u}\left(\frac{\mathcal{N}_{* h, u}}{\mathcal{N}_{* h, l}}-1\right) A_{\mathrm{sim}}} .
$$


Substituting $\mathcal{N}_{a u}=6.1, t_{a}=18.5 \mathrm{Myr}, \mathcal{N}_{* h, u}=5900$ and $\mathcal{N}_{* h, l}=1$ (see $\S 3.2$ ), we obtain $\Delta t_{a}=514\left(A_{\text {eff }} / A_{\text {sim }}\right)$ yrs. At the solar circle $A_{\text {eff }}=530 \mathrm{kpc}^{2}$ and we simulate a disk area $A_{\text {sim }}=5 \mathrm{kpc}^{2}$, so that an association forms in $A_{\text {sim }}$ every $5.5 \times 10^{4}$ years.

In the simulation, the position of each association is taken to be completely random in the plane $(x, y)$, but in the vertical direction $(z)$ the random distribution is weighted by a Gaussian profile of scale height $H_{*}=85 \mathrm{pc}$. The masses of the stars in each generation of each association are chosen using equations (A1) and (A2). The adopted gas distribution from which we derive the dust distribution is given by equation (24), and the methods for including dust absorption and scattering are given in Appendix B. To avoid boundary effects, the evolution of $U_{\text {band }}$ is studied only in the central part of $A_{\text {sim. }}$.

Figure 7 shows the time evolution of $U_{\mathrm{FUV}}$ at a point located in the center of simulation area and in the galactic plane. The radiation field energy density $U_{-17}$ is expressed in units of $10^{-17} \mathrm{erg} \mathrm{cm}^{-3} \AA^{-1}$. We note that Habing (1968) found that the local interstellar radiation field is approximately $U_{\mathrm{FUV}}^{\mathrm{H}}=4.6 \times 10^{-17} \mathrm{erg} \mathrm{cm}^{-3} \AA^{-1}$. The first association in the simulation volume forms at $t_{\text {sim }}=0$. Although difficult to see in Figure 7, it takes $\sim 40$ Myr to build up the FUV-ISRF once star formation commences. After this time, a "steady state" is achieved with large time fluctuations of $U_{-17}$. In addition, large spatial gradients are derived when $U_{-17}$ is evaluated at different points in the plane at a given instant of time. Note that although the probability is small, a massive star can form in a small association (see Appendix A). These small associations are abundant, and many of the HII regions encompassing the point are produced by these unusually massive stars in small associations.

The differences in the behavior of the radiation field in the $\mathrm{H}_{2}$ band $[912-1100 \AA]$ and in the FUV $-\mathrm{H}_{2}$ band $[1100-2070 \AA]$ are shown in Figures 8a,b. Note the high concentration of points around the median value, which corresponds to $U_{\mathrm{H} 2} \sim U_{\mathrm{FUV}-\mathrm{H} 2}$. The ordered sequence of points outside the crowded region in Figure $8 \mathrm{~b}$ corresponds to periods of time when the radiation field is completely dominated by one association. In Figure 8b, two larger excursions to high values of $U$ have been connected by lines in order to show how the field evolves as generations form and stars die. The large fluctuation that starts at $t_{\text {sim }}=1875 \mathrm{Myr}$ is produced by an association at 190 pc with 2830 high-mass stars. The fluctuation that starts at $t_{\text {sim }}=3975 \mathrm{Myr}$ is produced by an association at $32 \mathrm{pc}$ with 440 high-mass stars. The main trend seen in Figure $8 \mathrm{~b}$ is that, as $U_{\mathrm{FUV}}$ increases, the ratio $U_{\mathrm{H}_{2}}$ to $U_{\mathrm{FUV}-\mathrm{H}_{2}}$ increases. This expected correlation arises because high $U_{\mathrm{FUV}}$ is often associated with massive (and therefore hot) nearby stars.

Figure $8 \mathrm{c}$ shows the fraction of $U_{\text {band }}$ in the $[1100-2070 \AA]$ band that comes to the observer as scattered light (see Appendix B), as function of $U_{-17}$. The median value of $U_{\text {scatt }} / U_{\text {tot }}$ is $\sim 0.18$. We discuss this median value in comparison with observational data in $\S 5$.

Figure 8d shows for the FUV band the fraction of total field $U_{-17}$ that comes to the observer from the source that provides the larger contribution. For values of $U_{-17}$ around the median $(\sim 7)$ the dominant source provides in average $20 \%$ of the energy density $U_{-17}$, but the values range 
from $10 \%$ to $50 \%$. For large values of $U_{-17}$ the radiation field is totally dominated by only one source. The distribution of points in Figure $8 \mathrm{~d}$ indicate the degree of anisotropy of the field as function of $U_{-17}$.

In order to calculate the mean and median values of the FUV radiation field in the plane, a very long simulation was performed with $t_{\text {sim }} \sim 57$ Gyr. Excluding the first 400 Myrs, Figure 9a shows the corresponding distribution of the "fraction of occurrence" of $U_{\text {band }}$ values. The fraction of occurence is defined as $-d P(>U) / d \ln U$, where $P(>U)$ is the fraction of the simulation time that the point has an FUV energy density exceeding $U$. The fraction of occurrence can be thought of approximately as the probability of lying within a factor of 1.6 of $U_{\text {band }}$. The arrows indicate the mean and median values of the distributions. The two arrows for each mean value indicate the value including (the higher) or omitting (the lower) the time steps when the point is inside an HII region. The difference with and without HII regions is very small for the median because a given point is in an HII region only about $2 \%$ of the time. The difference for the mean is larger because $U_{\text {band }}$ is very high when the point is in a HII region. The expected mean value of $U_{\text {band }}$ (including HII regions) from equation 34 and Figure 6 at 57 Gyr of simulation is $\sim 0.9$ of the theoretical asymptotic value calculated in $\S 4.1$. Therefore, even when 57 Gyr of simulation are considered, the simulation does not provide an accurate estimate of the mean values because extremely rare events with large associations very close to the point in question tend to be missing. On the other hand, the mean value of $U_{\text {band }}$ excluding HII regions is closer to its asymptotic value since regions of space very near the association are excluded by the requirement that the point be outside the HII region. Note that the energy density in the FUV band is never lower than $U_{\mathrm{FUV}}$, min $=2.4 \times 10^{-17} \mathrm{erg} \mathrm{cm}^{-3} \AA^{-1}$; in the $H_{2}$ band the minimum value is $1.5 \times 10^{-17} \mathrm{erg} \mathrm{cm}^{-3} \AA^{-1}$. These minimum values correspond to the periods of time when no young associations are present in the central region of the simulation volume.

Figure $9 \mathrm{~b}$ shows the probability $P(>U)$ that, at a given instant in the simulation, the FUV energy density is greater than $U$. As stated above, the energy density in the FUV band is never lower than $U_{\mathrm{FUV}}$, min in the simulation, and therefore $P\left(>U_{\mathrm{FUV}, \min }\right)=1$. The curves labeled 1 Gyr, 4 Gyr, and 56 Gyr, correspond to the probabilities $P(>U)$ calculated from simulations extending over time intervals $\Delta t=1 \mathrm{Gyr}, 4 \mathrm{Gyr}$, and $56 \mathrm{Gyr}$, respectively. Note that the peak energy density decreases as $\Delta t$ drops. Even for a simulation time of 56 Gyr, events with $U_{-17}>4000$ are missing. For $U_{-17}>10$, the probability $P(>U)$ can be fitted by the power law $P(>U) \simeq 10 U_{-17}^{-3 / 2}$. For $U_{-17}>300$ a better fit is $P(>U) \simeq 15 U_{-17}^{-3 / 2}$. This dependence arises from the fact that the volume of the sphere around a source of luminosity $\mathcal{L}$ where the energy density exceeds $U$ is proportional to $(\mathcal{L} / U)^{3 / 2}$. The volume filling factor $\phi(>U)$ of these spheres therefore scales as $U^{-3 / 2}$. For $\phi<<1$, we have $P(>U)=\phi(>U)$, so that in this case $P(>U) \propto U^{-3 / 2}$. This relation is not exact because, for example, extinction is neglected, and the overlapping of spheres from different sources is not considered. However, the fit becomes better at high values of $U$ since these effects become unimportant there, since the volume around the sources is then small and consequently overlapping and extinction can be neglected. Figure $9 \mathrm{~b}$ also 
shows the typical time $t_{U}$ (right vertical axis) between two events that raise the energy density to $>U$ at a fixed point in space. It can be shown (Hollenbach et al. 2002) that $t_{U}=t_{\mathrm{FUV}} / \phi(>U)$, where $t_{\mathrm{FUV}}$ is the typical time that a source (an association) emits strongly in the FUV band (see

Fig. 4). The power law $t_{U}=t_{\mathrm{FUV}} / P(>U)=2 U_{-17}^{3 / 2} \mathrm{Myr}$ assumes $t_{\mathrm{FUV}}=20 \mathrm{Myr}$ and is good for $U_{-17}>10$. For $U_{-17}>300$ a better fit is $t_{U}=1.3 U_{-17}^{3 / 2}$ Myr. From this relation one can see that for $t_{\text {sim }}=57$ Gyr only the events with $U_{-17}<1000$ are well sampled.

For the FUV band, Figure 10 shows the evolution of the mean $\langle U\rangle$ and the median $U_{\text {med }}$ radiation fields (including HII regions) normalized to the theoretical value $\langle U\rangle_{\infty}$ derived in the previous section. The calculation of these averages is over the period of time $t_{\text {sim }}$ starting at time zero when associations begin to form in the simulation area. The theoretical ratio $\langle U\rangle_{t_{\text {sim }}} /\langle U\rangle_{\infty}$ (plotted in Fig. 6) is also shown in Figure 10 for comparison. Note that the median initially grows for $\sim 200$ Myr and then stabilizes, showing that the simulation is very efficient for calculating the median value of the field. The amplitude of fluctuations tends to decrease with time since the averages are made over the entire history of the simulation. The large fluctuation in $\left\langle U_{\text {sim }}\right\rangle /\langle U\rangle_{\infty}$ at $\sim 100 \mathrm{Myr}$ is due to an association of $\sim 300$ high-mass stars (the most massive star with a mass of $86 M_{\odot}$ ) born at $t_{\mathrm{sim}} \sim 60 \mathrm{Myr}$ at a distance of $\sim 120 \mathrm{pc}$ (with $z \sim-80 \mathrm{pc}$ ) from the central point. At $t_{\mathrm{sim}} \sim 75 \mathrm{Myr}, U \sim 25 \times 10^{-17} \mathrm{erg} \mathrm{cm}^{-3} \AA^{-1}$, mainly due to the FUV flux from this association. The second large fluctuation at $\sim 600 \mathrm{Myr}$ is due to an association of $\sim 41$ high-mass stars born at $t_{\mathrm{sim}} \sim 540 \mathrm{Myr}$ at a distance of $\sim 30 \mathrm{pc}$ (with $z \sim+23 \mathrm{pc}$ ) from the central point. This association is relatively small, but its most massive star has a mass of $114 M_{\odot}$. At $t_{\text {sim }} \sim 560 \mathrm{Myr}, U \sim 80 \times 10^{-17} \mathrm{erg} \mathrm{cm}^{-3} \AA^{-1}$, mainly due to the FUV flux from this star. The large fluctuation at $\sim 21$ Gyr is due to a very rare event: an association of 3120 high-mass stars at a distance of $\sim 35 \mathrm{pc}$ (with $z \sim-9 \mathrm{pc}$ ) that raises the FUV radiation field to a peak value of $U_{-17} \sim 3200$. The radiation field has $U_{-17}>1000$ for over $20 \mathrm{Myr}$, enough to raise $\left\langle U_{\text {sim }}\right\rangle$ over $\langle U\rangle_{\infty}$ even when the average is made over $21 \mathrm{Gyr}$.

As stated in $§ 3.3$, the star formation rate is expected to fluctuate during the simulation. Figure 11 shows the evolution of $\varsigma_{* h}$, the surface (number) density of high-mass stars in the simulation. The thick line corresponds to the average surface density over $A_{\text {sim }}$ (i.e. $5 \mathrm{kpc}^{2}$ ), whereas the thin line corresponds to the average within $500 \mathrm{pc}$ from the central point. As expected, the fluctuations are larger for the surface density within $500 \mathrm{pc}$. For a simulation interval between 0.1 to $57 \mathrm{Gyr}$, the mean value of $\varsigma_{* h}$ over $A_{\mathrm{sim}}$ is $\sim 540 \mathrm{kpc}^{-2}$, and within $500 \mathrm{pc}$ is $\sim 530 \mathrm{kpc}^{-2}$. Due to the large simulation time, these mean values of $\varsigma_{* h}$ are similar and correspond to a SN II every 62 yrs in the whole galaxy (we take $A_{\text {eff }}=530 \mathrm{kpc}^{2}$ ).

\section{The Local FUV Radiation Field: Observations and Theory}




\subsection{The Diffuse FUV Radiation Field}

Observationally, it has been established that diffuse radiation coming from regions where no sources are observed (i.e. far from the regions of recent star formation) is a minor component of the FUV-ISRF. This diffuse component arises primarily from large angle scattering by dust grains of FUV photons from Galactic sources, with a smaller contribution from extragalactic sources. Measurements of the diffuse FUV radiation field from these regions place an upper limit of $200-400$ photons $\mathrm{s}^{-1} \mathrm{sr}^{-1} \AA^{-1}$ in the $[912-1200 \AA]$ band (Murthy et al. 1991; Bowyer 1991; Henry et al. 1980). This upper limit corresponds to a radiation density $U_{\text {diffuse }} \sim 1.7-3.4 \times 10^{-18} \mathrm{erg} \mathrm{cm}^{-3} \AA$ and represents less than $10 \%$ of the estimates of local FUV energy density. By comparing a map resulting from summing the fluxes from individual FUV bright stars (Gondhalekar, et al. 1980) to a map constructed from large angle detections, Henry (1991) demonstrated that the FUV radiation field is dominated by the direct (or forward scattered) flux from relatively nearby FUV-producing stars. In the previous section we presented model results that showed that the median value of $U_{\text {scatt }} / U_{\text {tot }}$ is $\sim 0.18$, which is higher than the above estimates of $U_{\text {diffuse }} / U_{\text {tot }} \leq 0.1$. This difference arises because the "diffuse field" $U_{\text {diffuse }}$ comes from regions in the sky far from strong stellar sources, whereas the scattered field $U_{\text {scatt }}$ refers to all scattered flux, including that scattered through small angles. In fact, we find (Appendix B) that about 1/3 of the once-scattered radiation from a source comes from lines of sight within a cone of five degrees from the observer to the source. Taking this into account, we conclude that our estimate of the scattered flux is consistent with the observed diffuse field.

\subsection{The Observed FUV Radiation Field in the Solar Vicinity}

Neglecting diffuse radiation, the spectral energy density $U_{\lambda}$ at wavelength $\lambda$ in the immediate solar vicinity can be directly measured by adding the observed flux $F_{\lambda}(i)$ of all the FUV stellar sources $i$. By including all the data in the S2/68 Survey (more than 50,000 stars), Gondhalekar et al. (1980) estimated the flux at several wavelengths in the FUV band. At $1400 \AA$ the estimated energy density is about $5 \times 10^{-17} \mathrm{erg} \mathrm{cm}^{-3} \AA^{-1}$. Habing (1968), like Gondhalekar, added the fluxes from individual stars to estimate $U_{\lambda}$ in the immediate solar neighborhood. The estimated value for $U_{1400 \AA}$ was $4.2 \times 10^{-17} \mathrm{erg} \mathrm{cm}^{-3} \AA^{-1}$ when the direct light from all the bright stars B9 and earlier were included. For the extreme case of albedo 0.9, the Habing's value with diffuse light included

was $U_{1400 \AA}=7.5 \times 10^{-17} \mathrm{erg} \mathrm{cm}^{-3} \AA^{-1}$. However, this value depended on the assumptions made to infer the FUV flux for each object from its visual magnitude $m_{V}$ and its color excess $E(B-V)$. Jura (1974) recomputed $U_{\lambda}$ near $1000 \AA$ and obtained a value $\sim 7 \times 10^{-17} \mathrm{erg} \mathrm{cm}^{-3} \AA^{-1}$ by using updated calculations of stellar atmospheres and including all stars B5 and earlier listed in the Catalogue of Bright Stars. Utilizing a different technique, Henry et al. (1980) have directly measured the local $U_{\lambda}$ by taking large beam $\left(12^{\circ} \times 12^{\circ}\right)$ observations over about one third of the sky and extrapolating to obtain the unobserved portion. Henry et al. found that the average energy density is $5.8 \times 10^{-17} \mathrm{erg} \mathrm{cm}^{-3} \AA^{-1}$ in the band [1180-1680 $\AA$ ], and $5.3 \times 10^{-17} \mathrm{erg} \mathrm{cm}^{-3} \AA^{-1}$ 
at $1400 \AA$. Draine (1978) compiled a number of the observations and models of that epoch, and did a fit to some of them (primarily Henry et al (1977), Witt and Johnson (1973), Belyaev et al (1971), and Jura (1974)) to obtain what has become another standard (besides Habing) field, sometimes called the "Draine field". Averaged over the FUV band, it is approximately 1.7 times higher than the Habing field, i.e., the Draine field has $G_{0}=1.7$. A summary of these estimates is given in Table 2 .

The asymmetry of nearby FUV point sources results in a non-isotropic local FUV field. Most, if not all, OB stars are born in associations, and there are few associations that are young and close enough to produce a significant contribution to the FUV field. A sample of the nearby OB associations reveal about 7 associations within a kiloparsec of the Sun (see §3.3). The distribution of their distances above and below the galactic plane is quite symmetric, but their distribution in the plane is totally asymmetric. When the observed FUV field is plotted in Gould coordinates (Galactic Coordinates tipped by about $19^{\circ}$ ), a clear excess toward the active section of the Gould Belt is apparent (Henry et al. 1980). Even when our simulations do not consider correlation between the positions where coeval associations born, the results in Fig 8d shows that because FUV sources form in groups, FUV field is in general non-isotropic, in particular when the radiation field is high.

\subsection{The Mean and Median FUV Radiation Field at the Solar Circle}

Complementing these more or less direct determinations of $U_{\lambda}$ in the immediate solar neighborhood are the estimates, such as ours, obtained by modeling a spatial distribution of the relevant spectral type stars and the dust. The advantages of modeling are that it corrects for the incompleteness in the catalogs, it is useful to quantify the variations from different distributions, and it generalizes to other locations than the immediate solar neighborhood. The disadvantage is the necessity to introduce simplifying assumptions such as smooth dust distributions and prototype stellar luminosities. However, the "direct" procedure is only useful to give a snapshot of the local FUV-ISRF at the current time, whereas the theoretical models permit the study of the temporal and spatial behavior of the UV field in the interstellar medium, which is the main subject of the present paper.

In the previous section, the mean FUV field was calculated assuming the model ingredients, but, for the sake of comparison, we reproduce here one of the classical calculations of Habing. The "continuous approximation" in the Habing (1968) model includes the contribution of 10 associations and 577 field stars between B0 and B5. The FUV emission of these sources is assumed to be uniformly distributed in the plane (the field stars in $1 \mathrm{kpc}^{2}$ and the associations within $1 \mathrm{kpc})$. The distribution is proportional to $\exp \left[-(z / 120 \mathrm{pc})^{2}\right]$ in the vertical direction. Habing assumed a homogeneous plane-parallel layer of dust of thickness 150 pc producing an extinction of $3.5 \mathrm{mag} \mathrm{kpc}^{-1}$. By integrating over this continuous medium (there are neither stars nor associations, but an equivalent emitting medium), he obtained what we term the observed-source 
mean radiation energy density at $1400 \AA$ of $\left\langle U_{1400 \AA}\right\rangle=3.4 \times 10^{-17} \mathrm{erg} \mathrm{cm}^{-3} \AA^{-1}$. (This value assumes that the dust has zero albedo, so there is no scattered light. For an albedo of 0.9, Habing obtain $\left.\left\langle U_{1400 \AA}\right\rangle=5.6 \times 10^{-17} \mathrm{erg} \mathrm{cm}^{-3} \AA^{-1}\right)$. The distinction between Habing's observed-source mean and our mean is that ours is a mean based on the star formation rate in the entire Galaxy, whereas Habing's is based on the observed FUV sources in the solar vicinity. The surface emissivity $\Sigma_{L_{\mathrm{FUV}}}$ in Habing's model is about $1 / 3$ of the value in our model; this is plausible, since we expect his value to be closer to our typical value than to our mean (§3.3). Because his dust opacity is somewhat higher than ours, his observed-source mean radiation density is smaller yet, about $1 / 4$ of the value of $\left\langle U_{\mathrm{FUV}}\right\rangle$ calculated in $\S 4.1$.

The observed-source mean FUV energy density estimated by Habing (1968) and the mean FUV energy density calculated in this paper are summarized in Table 3, while the typical values are summarized in Table 4. Habing's typical value is different from ours: his estimate is obtained by excluding the space in the neighborhood of associations (about $10 \%$ of the total volume) and taking the average over the remaining space. As discussed in $\S 4$, we estimate the typical FUV radiation by calculating median value of the FUV field at a point in a large simulation of the model. Compared to the band-averaged FUV "Habing field", our model results give $G_{0}=(1.6,3.1)$ for the (median, mean) radiation field at the solar circle. Table 4 also gives the value below and above which the FUV energy density exists $20 \%$ of the simulation time. A more complete characterization of the FUV radiation field is given by the probability distribution in Figure 9 . This representation obscures the temporal correlations of the radiation field, however, so it is also useful to use the FUV field history shown in Figures 7 and $8 \mathrm{a}$ to characterize the typical behavior of the FUV field.

\section{Effect of Runaway OB Stars and Supernova}

\subsection{Runaway OB Stars}

"Runaway" OB stars, as defined by Blaauw (1961), are OB stars with peculiar space velocities that exceed $30 \mathrm{~km} \mathrm{~s}^{-1}$ and that are directed away from known clusters and associations. About $10-30 \%$ of $\mathrm{O}$ stars and $5-10 \%$ of B stars are observed to be runaways. These runaway stars have large space velocities (30-200 $\mathrm{km} \mathrm{s}^{-1}$ ), and most of them appear to be single (Gies 1987; Stone 1991; Hoogerwerf et al. 2000a,b). The most viable scenarios for the formation of runaway stars are: (i) a supernova explosion in a massive binary (Zwicky 1957; Blaauw 1961), in which the primary becomes a compact object and the secondary moves away with a velocity comparable to its pre-explosion orbital velocity; and (ii) dynamical ejection from a dense stellar cluster (Poveda et al. 1967; Gies \& Bolton 1986), in which the most efficient interaction is the encounter of two hard binary systems. Recently, milli-arcsecond astrometry provided by Hipparcos and radio observations have provided specific evidence for both scenarios (Hoogerwerf et al. 2000a,b), but the relative importance of the two mechanisms remains unknown. 
Since the OB stars produce the FUV radiation field, and a non-negligible fraction of them are runaways, we must estimate their effect on the evolution of the FUV-ISRF. We assume that about $20 \%$ of $\mathrm{O}$ stars and $7 \%$ of B stars in each generation are ejected in random directions with a velocity weighted by the probability distribution

$$
p(v) \propto v \exp \left[-\left(\frac{v-v_{c}}{\sigma}\right)^{2}\right]
$$

with $v_{c}=\sigma=30 \mathrm{~km} \mathrm{~s}^{-1}$, appropriate for runaways. We consider only stars with masses larger than $8 \mathrm{M}_{\odot}$; i.e. $\mathrm{O}$ and early B stars. Our primary assumption is that OB-star runaways (regardless of the ejection mechanism) are ejected at the moment they form. In the dynamical ejection scenario, the runaways are ejected very early in the cluster evolution, so that this assumption is well justified. In the binary-supernova scenario, the creation of the runaway is delayed until the explosion of the more massive component of the binary system. Therefore, the instantaneous ejection assumption overestimates the size of the sphere of influence of the SN-ejected runaways and provides an upper limit on the effect of runaways.

To estimate the effect of runaway stars on the FUV radiation field, we compute the difference $\Delta U_{-17 \text {,run }}$ of the radiation energy density produced by the stars with masses over $8 \mathrm{M}_{\odot}$ in two cases, with and without runaways. Figure 12 shows the effects of including runaways in the simulation performed in $\S 4.2$; that is, the simulation parameters are exactly those used to generate the time evolution of the FUV-ISRF in Figures 7 and 8, so that in both simulations the associations are formed with the same size, and at the same place and time. Additionally, we have assumed periodic boundary conditions; runaway stars that leave the simulation area from one boundary are reinserted at the opposite boundary.

The heavy line in Figure 12 shows $\Delta U_{-17, \text { run }}\left(t_{\text {sim }}\right)$ for the same time interval in Figure 8a. The dotted line shows the total energy density $U_{\mathrm{FUV}}$ from the simulation in $\S 4.2$ (i.e. considering stars of all masses and assuming that they all remain at their birth places). On average, at a given moment of time, there are about 200 runaway stars in the simulation area $(\sim 40 \mathrm{O}$ runaways and $\sim 160$ early $\mathrm{B}$ runaways in $5 \mathrm{kpc}^{2}$ ), but very few of them make a passage close to the observer and create a spike in the radiation field. Most of these spikes are produced by nearby associations; in the plot, only one significant spike is produced by a runaway coming from a distant (844 pc) association. For FUV spikes characterized by peak strengths $U_{-17}$, the duration of the spike is of order $\Delta t_{U} \sim 10^{6} L_{5}^{1 / 2} v_{7}^{-1} U_{-17}^{-1 / 2}$ years, where $L_{5}=L_{F U V} / 10^{5} L_{\odot}$ is the stellar luminosity and $v_{7}=v / 100 \mathrm{~km} \mathrm{~s}^{-1}$ is the runaway speed. Spikes that dominate the FUV field occur roughly once every $\sim 100$ Myr. The negative values of $\Delta U_{-17 \text {,run }}$ are also produced by nearby associations, since on average their runaway stars are at greater distances from the point in question than is the natal association.

The median value of $\Delta U_{-17 \text {,run }}$ in the simulation is 0.016 (this slightly positive value is due to the fact that it is assumed that the parent cloud does not absorb radiation from the runaways). The ratio of the fluence in the spikes to that in the rest of the radiation field is $\sim 0.025$. Therefore, 
the conclusions drawn in previous sections (assuming that all the stars remain at their place of birth) do not change significantly if runaways are considered. However, very sporadically, the FUV-ISRF is dominated by the radiation from a runaway. These dominant spikes tend to occur when $U_{\text {FUV }}$ is high (i.e., there are nearby associations).

\subsection{Supernovae}

Supernovae produce very large FUV luminosities for very short periods of time. Core-collapse supernovae produce UV radiation by two separate phenomena. One is the FUV radiation burst due to shock breakout, and the second is the FUV emission associated with the visible supernova. Scalo et al. (2001) estimate that each process typically produces about $10^{47}$ erg in UV photons, over time intervals of less than an hour and $2-3$ months, respectively. The luminosities in these phases are very large compared with the mean FUV luminosity per main sequence high-mass star (i.e. $\left\langle L_{\mathrm{FUV}}\right\rangle_{h}=5.8 \times 10^{4} L_{\odot}$, see $\S 2.2$ ) However, the luminosity-weighted lifetime of main sequence high-mass stars (i.e. $\left\langle t_{\mathrm{FUV}}\right\rangle=7.8 \times 10^{6} \mathrm{yr}$ ) is much longer than the short periods where SN are actively emitting FUV photons, and as a result the total FUV energy emitted per main sequence

high-mass star $\left(\left\langle L_{\mathrm{FUV}}\right\rangle_{h} \times\left\langle t_{\mathrm{FUV}}\right\rangle \sim 6 \times 10^{52} \mathrm{erg}\right)$ is about five orders of magnitude larger than the FUV fluence per SN ( few $\left.10^{47} \mathrm{erg}\right)$; indeed, the emitted FUV energy is about 60 times larger than the typical total energy released by a SN $\left(\sim 10^{51} \mathrm{erg}\right)$. Therefore, SN FUV spikes make a negligible contribution to the median and mean values of the radiation field calculated in previous sections.

However, as pointed out by Scalo et al. (2001), these FUV spikes may be relevant for atmospheric chemistry and for inducing mutations in living creatures. Scalo et al. (2001) calculate the frequency of events exceeding the solar flux in the $2000-3000 \AA$ at 1 AU and conclude that such events have occurred several times during the history of the Earth. Scalo et al. (2001) have considered an homogeneous distribution of SN. If as in the present work, massive stars are assumed to form in associations, high $U$ events tend to occur in groups because high mass members of a given association explode within a relatively short period of time and at similar distances. Then, the time intervals between events (in different groups) exceeding a given flux increase by a factor $\dot{\zeta}_{* h} / \dot{\zeta}_{a}(>1)=\ln \mathcal{N}_{* h, u}=8.7$. In addition, we note that the spectrum of the shock breakout is dominated by extreme ultraviolet and soft X-ray emission (Matzner \& McKee 1999), so only a small fraction of the emission is in the FUV band. When this energetic radiation strikes the Earth's atmosphere, much of it will be reprocessed to lower energies. The analysis of this process and of the effects on atmospheric chemistry is beyond the scope of the present work. 


\section{Conclusions}

The presence of a time and space-dependent FUV field may have a major impact on the structure of the neutral interstellar medium. In particular, the main process of $\mathrm{H}_{2}$ and $\mathrm{CO}$ destruction is photodissociation by radiation in the $\mathrm{H}_{2}$ band (912-1100 $\AA$ ), and the primary heating process of the neutral interstellar gas is grain photoelectric heating by radiation in the FUV band (912-2070 ̊). The FUV controls the amount of interstellar material in the cold (CNM) and warm (WNM) neutral phases.

In this paper, we have presented a study of the temporal behavior of the radiation field in these bands, as well as at $1400 \AA$. To simulate the evolution of the radiation field at the solar circle of the Milky Way, we have adopted: a) a power-law initial mass function with an upper mass cutoff for high-mass stars in associations (§2.1); b) a stellar-mass dependence of the FUV and $\mathrm{H}_{2}$ band luminosities, and the main sequence lifetime for stars of various masses ( $(2.2)$; c) a stellar-mass dependence of the ionizing luminosity from high-mass stars (§2.3), which, coupled with radio observations of the total ionizing luminosity in the Galaxy, determines the rate of high-mass star formation $(\S 2.4)$; d) a history of the star formation rate in an association ( $(3.1)$; e) a dependence of the birth-rate of associations on the number of high-mass stars $\mathcal{N}_{* h}$ in the association (§3.2); and f) a vertical distribution of associations and dust ( 44 ). Our calculations are based on the distribution of OB associations in the Galaxy as determined by MW97 and are completely independent of observations of the local interstellar radiation field.

The most important conclusions are the following:

1) We have computed the typical (or median) value of the FUV energy density at the solar circle in various bands and at $1400 \AA$. Our computed value of the median FUV energy density at $1400 \AA$ at the solar circle $\left(\sim 7.5 \times 10^{-17} \mathrm{erg} \mathrm{cm}^{-3} \AA^{-1}\right)$ compares reasonably well with the currently measured local value at $1400 \AA\left(5.3 \times 10^{-17} \mathrm{erg} \mathrm{cm}^{-3} \AA^{-1}\right.$; Henry et al. 1980) and with Habing's and Draine's recommended values for the typical local interstellar medium $\left(\sim 5 \times 10^{-17}\right.$ and $\sim 9.8 \times 10^{-17} \mathrm{erg} \mathrm{cm}^{-3} \AA^{-1}$, respectively). The observationally estimated local value at 1400 $\AA$ is near the most probable FUV energy density values in the simulation (Fig. 9). Therefore, the local neighborhood has neither an exceptional high or low number of OB associations. We find band-averaged median energy densities of $(7.4,7.1,7.2) \times 10^{-17} \mathrm{erg} \mathrm{cm}^{-3} \AA^{-1}$ for the $([912-1100$ $\AA],[1100-2070 \AA],[912-2070 \AA])$ bands, respectively. Our value for the FUV band is 1.6 times the value recommended by Habing $\left(G_{0}=1.6\right)$, which is close to Draine's (1978) suggested value of $G_{0}=1.7$.

2) MW97 proposed a distribution for the birth rate of associations in terms of the number of high-mass stars in the association, $d \dot{\mathcal{N}}_{a} / d \ln \mathcal{N}_{* h} \propto 1 / \mathcal{N}_{* h}$, based on observations of giant radio HII regions. Here we have shown that this distribution produces a median value of the solar circle FUV-ISRF in agreement with the currently measured local value. This is consistent with the extension of the MW97 distribution to small associations, as they proposed. 
3) We have included scattering and absorption by interstellar dust. The absorption by dust limits the region that contributes to the FUV field at a given point to distances less than about 500 pc. The scattering produces a "diffuse" field that is observed to be less than $10 \%$ of the total FUV field. Our models are consistent with these observations. Thus, most of the FUV energy density at a given point at the solar circle comes from the sum of discrete sources.

4) We have computed the average (or mean) of the FUV energy density in various bands and at $1400 \AA$. The mean value is nearly twice the median value, due to infrequent spikes in the radiation field. The mean energy density at $1400 \AA$ is $14.4 \mathrm{erg} \mathrm{cm}^{-3} \AA^{-1}$. The band-averaged mean energy densities are $U_{-17}=(15.7,13.8,14.2)$ for the $([912-1100 \AA],[1100-2070 \AA],[912-2070$ A]) bands, respectively.

5) The fluctuations in the $\mathrm{H}_{2}$ band [912-1100 $\AA$ ] are larger than those in the FUV band, and the ratio of the mean to median energy density in this band is larger than that in the FUV band. This is both because the extinction in the $\mathrm{H}_{2}$ band is larger than in the FUV band and because the sources contributing to the $\mathrm{H}_{2}$ band are rarer than the those contributing to the FUV band.

6) The FUV field undergoes substantial fluctuations, with the larger ones being less frequent. Figures 7 and 8 show that at the solar circle the energy density of the radiation $U$ fluctuates above the median value by a factor 2 to 3 every $100-200$ Myr. For $U>>U_{\text {med }}$, the time interval $t_{U}$ between episodes with fields greater than $U$ is roughly given by $t_{U} \simeq 2 U_{-17}^{3 / 2}$ Myr or $2 U_{-15}^{3 / 2}$ Gyr (Figure 9). These infrequent epochs of high fields last of order $30 \mathrm{Myr}$, the lifetime of the nearby $\mathrm{OB}$ association that creates the high field. As we shall show in a subsequent paper, these fluctuations affect the relative fractions of cold neutral medium and warm neutral medium, and thereby create a fluctuating state of the ISM as a function of space and time.

7) A point at the solar circle is inside the $\mathrm{H}$ II region of a nearby OB association about $2 \%$ of the time, based on the assumption that the size of the H II region is that of a Strömgren sphere in a medium with a density of $n=1 \mathrm{~cm}^{-3}$.

8) In general, a significant fraction of the FUV radiation field comes from a single source. For values of $U_{-17}$ around the median $(\sim 7)$ the dominant source provides on average $20 \%$ of the energy density, but the values range from $10 \%$ to $50 \%$. For large values of $U_{-17}$, the radiation field is totally dominated by only one source. Therefore, the FUV field is asymmetric at a given point, the asymmetry growing for higher fields. Very low values of the radiation field are very infrequent - indeed, values below 1/3 of the median FUV field are totally absent from our simulations.

9) OB runaways produce short-lived spikes in the radiation field. At a given moment of time at the solar circle, we estimate that there are about 40 runaway stars per $\mathrm{kpc}^{2}(\sim 8 \mathrm{O}$ runaways and $\sim 30$ early $\mathrm{B}$ runaways), but very few of them make a passage close to the observer and create a spike in the radiation field. However, very sporadically (on the order of once every $\sim 100$ Myr, see Figure 12), the FUV-ISRF is briefly dominated by the radiation from a runaway. These dominant spikes tend to occur when $U_{\mathrm{FUV}}$ is high (i.e., there are nearby associations). However, 
the duration of the spikes is short (about one Myr) and the total fluence in the spikes is negligible in comparison with the total fluence from the stars in the associations.

10) Supernovae raise the radiation field significantly above the median value every $\sim 5 \times 10^{4}$ years. However, their duration is so brief that the total fluence in the spikes is negligible in comparison with the total fluence from the stars in the associations.

We acknowledge support from the NASA Astrophysical Theory Program in RTOP 344-0410-02, which funds The Center for Star Formation Studies, a consortium of researchers at NASA Ames, University of California at Berkeley, and University of California at Santa Cruz. AP was supported as a Senior Associate for part of this research by the National Research Council and by the Universidad de Los Andes by CDCHT project C-1098-01-05-B. The research of CFM is supported in part by NSF grant AST-0098365. We thank Juan Mateu and Gustavo Bruzual for providing theoretical models of evolutionary spectra from high mass stars. 
TABLE 1

Stellar Properties

\begin{tabular}{|c|c|c|c|c|c|}
\hline Quantity & Units & \multicolumn{2}{|c|}{ Approximation } & \multicolumn{2}{|c|}{ Mass Range } \\
\hline \multirow[t]{5}{*}{ MS Life Time } & Myr & $7.65 \times 10^{3}$ & $m^{-2.80}$ & 1.2 & $-3 M_{\odot}$ \\
\hline & & $4.73 \times 10^{3}$ & $m^{-2.36}$ & 3 & -6 \\
\hline & & $2.76 \times 10^{3}$ & $m^{-2.06}$ & 6 & -9 \\
\hline & & $1.59 \times 10^{3}$ & $m^{-1.81}$ & 9 & $-\quad 12$ \\
\hline & & $7.60 \times 10^{2}$ & $m^{-1.57}+2.30$ & 12 & $-\quad 120$ \\
\hline \multirow[t]{8}{*}{$L_{\mathrm{H} 2}$} & $L_{\odot}$ & $1.98 \times 10^{-14}$ & $m^{26.6}$ & 1.8 & -3 \\
\hline & & $2.86 \times 10^{-8}$ & $m^{13.7}$ & 3 & -4 \\
\hline & & $1.35 \times 10^{-4}$ & $m^{7.61}$ & 4 & -6 \\
\hline & & $1.10 \times 10^{-2}$ & $m^{5.13}$ & 6 & -9 \\
\hline & & $1.07 \times 10^{-1}$ & $m^{4.09}$ & 9 & $-\quad 12$ \\
\hline & & $5.47 \times 10^{-1}$ & $m^{3.43}$ & 12 & $-\quad 15$ \\
\hline & & $9.07 \times 10^{0}$ & $m^{2.39}$ & 15 & $-\quad 30$ \\
\hline & & $9.91 \times 10^{1}$ & $m^{1.69}$ & 30 & $-\quad 120$ \\
\hline \multirow[t]{8}{*}{$L_{\mathrm{FUV}}-L_{H 2}$} & $L_{\odot}$ & $2.77 \times 10^{-4}$ & $m^{11.8}$ & 1.8 & -2 \\
\hline & & $1.88 \times 10^{-3}$ & $m^{9.03}$ & 2 & $-\quad 2.5$ \\
\hline & & $1.19 \times 10^{-2}$ & $m^{7.03}$ & 2.5 & -3 \\
\hline & & $1.47 \times 10^{-1}$ & $m^{4.76}$ & 3 & -6 \\
\hline & & $8.22 \times 10^{-1}$ & $m^{3.78}$ & 6 & -9 \\
\hline & & $2.29 \times 10^{0}$ & $m^{3.31}$ & 9 & - 12 \\
\hline & & $2.70 \times 10^{1}$ & $m^{2.32}$ & 12 & $-\quad 30$ \\
\hline & & $3.99 \times 10^{2}$ & $m^{1.54}$ & 30 & - 120 \\
\hline \multirow[t]{7}{*}{$s_{49}$} & $10^{49} \mathrm{phot} / \mathrm{s}$ & $2.23 \times 10^{-15}$ & $m^{11.5}$ & 5 & -7 \\
\hline & & $3.69 \times 10^{-13}$ & $m^{8.87}$ & 7 & $-\quad 12$ \\
\hline & & $4.80 \times 10^{-11}$ & $m^{7.85}$ & 12 & $-\quad 20$ \\
\hline & & $3.12 \times 10^{-8}$ & $m^{4.91}$ & 20 & $-\quad 30$ \\
\hline & & $2.80 \times 10^{-5}$ & $m^{2.91}$ & 30 & -40 \\
\hline & & $3.49 \times 10^{-4}$ & $m^{2.23}$ & 40 & -60 \\
\hline & & $2.39 \times 10^{-3}$ & $m^{1.76}$ & 60 & -120 \\
\hline
\end{tabular}


TABLE 2

The Local Energy Density

\begin{tabular}{lllc}
\hline \hline Authors & Method & Wavelength & $U\left[10^{-17} \mathrm{erg} \mathrm{cm}^{-3} \AA^{-1}\right]$ \\
\hline Habing 1968 & $\mathrm{A}$ & $1000 \AA$ & 3.3 \\
& & $1400 \AA$ & 4.2 \\
& & $2200 \AA$ & 2.2 \\
Jura 1974 & $\mathrm{B}$ & $1000 \AA$ & 7.0 \\
Gondhalekar et al. 1980 & $\mathrm{C}$ & $1000 \AA$ & 4.9 \\
& & $1400 \AA$ & 5.0 \\
Henry et al. 1980 & & $2200 \AA$ & 2.5 \\
& $\mathrm{D}$ & $1400 \AA$ & 5.3 \\
Draine 1978 & & $1180-1680 \AA$ & 5.8 \\
& $\mathrm{E}$ & $1000 \AA$ & 6.7 \\
& & $1400 \AA$ & 9.8 \\
& & $2200 \AA$ & 3.7 \\
\hline
\end{tabular}

Notes to Table 2

- Method A.- The FUV flux from bright stars B9 and earlier was inferred from its visual magnitude $m_{V}$ and its color excess $E(B-V)$.

- Method B.- Similar to method A but using updated stellar atmospheres model and including only bright stars B5 and earlier.

- Method C.- Similar to method B but including more than 50,000 stars in the S2/68 Survey.

- Method D.- Extrapolation from direct measurements of the spectrum in regions of $12^{\circ} \times 12^{\circ}$ over about one-third of the sky.

- Method E.- Values from the analytic expresion proposed by Draine (1978) for the "Standard UV background". His formula is based on estimates of the typical FUV energy density at the solar circle and in the solar neighborhood (Habing (1968); Gondhalekar and Wilson (1975); Witt and Johnson (1973) and Jura (1974)) and on local measurements (Hayakawa, et al. (1969); Belyaev et al. (1971) and Henry et al. (1977)). 
TABLE 3

The Mean Energy Density at the Solar Circle

\begin{tabular}{|c|c|c|c|}
\hline Authors & Method & Wavelength & $\overline{\left\langle\langle U\rangle\left[10^{-17} \mathrm{erg} \mathrm{cm}^{-3} \AA^{-1}\right]\right.}$ \\
\hline \multirow[t]{3}{*}{ Habing 1968} & $\bar{A}$ & $1000 \AA$ & $3.9-6.7$ \\
\hline & & $1400 \AA$ & $3.4-5.6$ \\
\hline & & $2200 \AA$ & $1.4-2.3$ \\
\hline \multirow[t]{4}{*}{ This paper } & B & 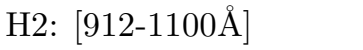 & 15.7 \\
\hline & & FUV-H2: [1100-2070Å] & 13.8 \\
\hline & & FUV: [912-2070Å] & 14.2 \\
\hline & & $1400 \AA$ & 14.4 \\
\hline \multirow[t]{4}{*}{ This paper } & $\mathrm{C}$ & 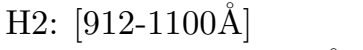 & $15.9-11.4$ \\
\hline & & FUV-H2: [1100-2070Å] & $12.1-9.8$ \\
\hline & & 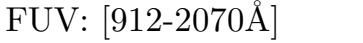 & $12.7-10.0$ \\
\hline & & $1400 \AA$ & $12.5-10.3$ \\
\hline
\end{tabular}

\section{Notes to Table 3}

- Method A.- "Continuous method" (see §5.3). Contribution of 10 associations and 577 field stars between B0 and B5. Left: direct star light; right: diffuse light included. Habing 1968, Table 6 and moderate extinction.

- Method B.- As method A but using the PDMF (see §4.1). Diffuse light included (see §6, Appendix B).

- Method C.- From simulation. Left (right) values include (exclude) HII regions. Diffuse light included (see Sec. 6, Appendix B). The quoted values are the averages over 5 points $280 \mathrm{pc}$ apart in a simulation of 57 Gyr. These mean values vary from point to point in one simulation and from one simulation to another (see text and Fig. 10). The variations of mean values excluding HII regions are much smaller. A point is inside an HII region about $2 \%$ of the simulation time. 
TABLE 4

The Typical Energy Density at the Solar Circle

\begin{tabular}{|c|c|c|c|}
\hline Authors & Method & "Wavelength & 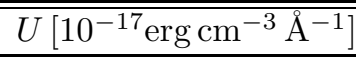 \\
\hline \multirow{3}{*}{ Habing 1968} & $\mathrm{~A}$ & $1000 \AA$ & $2.5<4.0<14.0$ \\
\hline & & $1400 \AA$ & $2.5<5.0<15.0$ \\
\hline & & $2200 \AA$ & $1.5<3.0<13.0$ \\
\hline \multirow[t]{4}{*}{ This paper } & median & H2: $[912-1100 \AA]$ & 7.4 \\
\hline & & 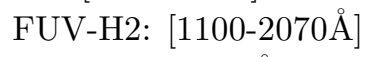 & 7.1 \\
\hline & & 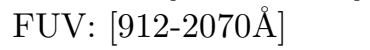 & 7.2 \\
\hline & & $1400 \AA$ & 7.5 \\
\hline \multirow[t]{4}{*}{ This paper } & $20 \%$ below & H2: $[912-1100 \AA]$ & 4.6 \\
\hline & & FUV-H2: [1100-2070Å] & 5.0 \\
\hline & & FUV: $[912-2070 \AA]$ & 5.0 \\
\hline & & $1400 \AA$ & 5.4 \\
\hline \multirow[t]{4}{*}{ This paper } & $20 \%$ above & H2: $[912-1100 \AA]$ & 14.8 \\
\hline & & 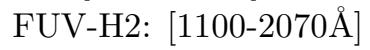 & 12.0 \\
\hline & & 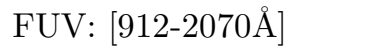 & 12.4 \\
\hline & & $1400 \AA^{2}$ & 12.5 \\
\hline
\end{tabular}

\section{Notes to Table 4}

- Method A.- Habing's 1968 recomended values for typical interstellar space near the solar circle (his Table 8 ). The three values given for each wavelength are the lower limit, the typical value, and the upper limit.

- Median.- Median value of the field in a point in a simulation with $t_{\text {sim }}=57$ Gyr. The first Gyr is discarded. See $\S 4.2$ and Figures 9 and 10.

- $20 \%$ above.- The value below which the energy density exists $20 \%$ of the simulation time.

- $20 \%$ over.- The value above which the energy density exists $20 \%$ of the simulation time. 
TABLE B1

Physical Properties of the Dust ${ }^{\mathrm{a}}$

\begin{tabular}{llll}
\hline \hline Wavelength & $\kappa_{a}$ & $\kappa_{s}$ & $g$ \\
\hline $912-1100 \AA$ & $1.910^{-21}$ & $8.9510^{-22}$ & 0.8 \\
$1100-2070 \AA$ & $8.010^{-22}$ & $7.510^{-22}$ & 0.75 \\
$1400 \AA$ & $7.0310^{-22}$ & $7.8110^{-22}$ & 0.756 \\
\hline${ }^{\mathrm{a}}$ Units of $\kappa$ are $\mathrm{cm}^{2}(\mathrm{H} \text { atom })^{-1}$ &
\end{tabular}




\section{A. MASS DISTRIBUTION IN ASSOCIATIONS}

The IMF in equation (3) is the probability distribution for the stellar masses in an association. Each realization of an association will have fluctuations about this distribution, with the fluctuations being larger for smaller associations. In order to make a realization for a single generation of an association that is consistent with the IMF, we set the masses of stars with $m_{i} \geq 2 M_{\odot}$ to

$$
m_{i}=\left[\mu_{i}^{-\Gamma}-x_{i}\left(\mu_{i}^{-\Gamma}-\mu_{i-1}^{-\Gamma}\right)\right]^{-1 / \Gamma} \quad\left(i=1, . ., \mathcal{N}_{*}\right)
$$

where

$$
\mu_{i}=\frac{m_{u}}{\left[\frac{i \phi_{h}}{\mathcal{N}_{* h} / 5}\left(\frac{m_{u}}{m_{h}}\right)^{\Gamma}+1\right]^{1 / \Gamma}}
$$

and $\mu_{0}=m_{u}$. We have assumed that there are 5 generations in an association, so that the generation has $\mathcal{N}_{* h} / 5$ stars. The mass $m_{1}$ of the most massive star in each generation is chosen at random by setting $x_{1}$ equal to a random number between 0 and 1 . The masses of the less massive stars are set assuming $x_{2}=x_{3}=\ldots=1 / 2$. This procedure enables us to recover the correct IMF slope near $m_{u}$ when a distribution of associations is considered. Since an important fraction of $L_{\mathrm{FUV}}$ and $L_{H_{2}}$ is emitted by the most massive star in the association, this approach also accounts for the significant differences that can be present in similar (i.e. same $\mathcal{N}_{* h}$ ) associations.

\section{B. DIRECT AND DIFFUSE RADIATION}

In this appendix we describe the procedure used to calculate the scattered radiation, allowing for the possibility that the source and/or the observer are out of the galactic plane. We assume that the absorbing and dispersing medium follows the HI vertical distribution (eq. 24). The geometrical parameters of the

problem are the height of the observer above the plane, $z_{o}$, the height of the emitter, $z_{e}$, and the projected separation between the two, $x_{o e}$, as indicated in Figure B1.

To calculate the contribution $d F_{\text {scatt }, 1}$ to the diffuse flux emitted at point 'e', scattered at point 'd', and observed at point ' $\mathrm{o}$ ', we erect a coordinate system based on the line of sight 'oe' from the observer to the source. Let 'p' denote the projection of 'd' onto this axis, $\varpi$ be the distance from 'p' to 'd', and $r_{e p}$ the distance from the source to 'p'. We then have

$$
d F_{\text {scatt }, 1}=\frac{L_{*}}{4 \pi r_{e d}^{2}} e^{-\tau_{e d}} d f_{\text {scatt }} \frac{\phi_{\text {scatt }}(\alpha, g)}{r_{d o}^{2}} e^{-\tau_{d o}} d \varpi \varpi d \theta
$$

where

$$
d f_{\mathrm{scatt}}=\kappa_{s} n_{\mathrm{H}}\left(z_{d}\right) d r_{e p}=\left[\frac{n_{\mathrm{H}}\left(z_{d}\right)}{n_{\mathrm{H}}\left(z_{p}\right)}\right] d \tau_{s}
$$

is the fraction of the incident flux scattered per unit area (measured normal to the axis 'oe') at ' $d$ '. In equation (B2), $\kappa_{s}$ is the grain scattering cross section per $\mathrm{H}$ atom, $\tau_{s}$ is the scattering optical depth along the direct ray 'oe', and $n_{\mathrm{H}}\left(z_{d}\right)$ is the number density of $\mathrm{H}$ atoms at height $z_{d}=z_{p}+\varpi \cos (\theta) \cos (\phi)$ (see Fig. B1) above the galactic plane. The term $\phi_{\text {scatt }}(\alpha, g)$ in equation (B1) is the scattering phase function; 
we adopt the Henyey \& Greenstein (1941) phase function

$$
\phi_{\text {scatt }}(\alpha, g)=\frac{1-g^{2}}{4 \pi\left(1+g^{2}-2 g \cos \alpha\right)^{3 / 2}},
$$

where

$$
g \equiv \frac{1}{4 \pi} \int d \Omega \cos \alpha \phi_{\text {scatt }}(\alpha, g) d \Omega
$$

is the average of the cosine of the scattering angle. The factors $\exp \left(-\tau_{e d}\right)$ and $\exp \left(-\tau_{d o}\right)$ in equation (B1) are the extinctions along the paths 'ed' and 'do', respectively, and include both absorption and scattering.

For the $912-1100 \AA$ and $1100-2070 \AA$ bands, and at $1400 \AA$, the adopted values for $\kappa_{a}, \kappa_{s}$ and $g$ are given in Table B1, and are derived from the data in Witt \& Gordon (1999) assuming that $\kappa_{a}=5.34 \times 10^{-22}\left(\tau / \tau_{V}\right)(1-a) \mathrm{cm}^{2}(\mathrm{H} \text { atom })^{-1}$ and $\kappa_{s}=5.34 \times 10^{-22}\left(\tau / \tau_{V}\right) a \mathrm{~cm}^{2}(\mathrm{H} \text { atom })^{-1}$.

The total flux $F_{\text {scatt }, 1}$ that arrives at 'o' after being scattered once at 'd' is simply the integration of $d F_{\text {scatt } 11}$ over the whole volume. In practice, the integration is done over the revolution volume around the axis 'oe' that satisfies $\alpha<\pi / 2$, because more than $96 \%$ of the diffuse flux comes from this volume. It is illustrative to compare $F_{\text {scatt }, 1}$ to the direct flux

$$
F_{\text {dir }}=\frac{L_{*}}{4 \pi r_{o e}^{2}} e^{-\left(\tau_{a}+\tau_{s}\right)}
$$

where $\tau_{a}$ is the absorption optical depth along the ray 'oe'. Taking advantage of the fact that the scattering is strongly peaked in the forward direction $(g \geq 0.75$ - Table B1), we normalize to the case of purely forward scattering $(g=1)$. In that case, the probability of a single scattering is $\tau_{s} \exp \left(-\tau_{s}\right)$ and $F_{\text {scatt }, 1}=F_{\text {dir }} \tau_{s}$. In the general case in which $g \leq 1$, we express the single scattering flux as:

$$
F_{\text {scatt }, 1}=F_{\text {dir }} \tau_{s} \mathcal{R} .
$$

We have evaluated $\mathcal{R}$ numerically in Figure B2. Note that it is close to unity, as expected from the fact that the scattering is strongly peaked in the forward direction.

The procedure described above considers the diffuse radiation arriving at ' $\mathrm{o}$ ' after only one scattering. To estimate the contribution of multiple scattering, we again consider the case of purely forward scattering. In this case, the probability that a photon will arrive at 'o' after $n$ scatterings is $\tau_{s}^{n} \exp \left(-\tau_{s}\right) / n$ !. We adopt the ansatz that the flux that arrives at 'o' after $n$ scatterings when $g<1$ is

$$
F_{\text {scatt }, n} \sim F_{\text {dir }} \frac{\left(\tau_{s} \mathcal{R}\right)^{n}}{n !}
$$

The total flux $F_{\text {tot }}$ received at 'o' (direct plus scattered) is then

$$
F_{\mathrm{tot}}=F_{\mathrm{dir}} e^{\tau_{s} \mathcal{R}}=\frac{L_{*}}{4 \pi r_{o e}^{2}} e^{-\tau_{a}-\tau_{s}(1-\mathcal{R})} .
$$

For purely forward scattering, $\mathcal{R}=1$ and scattering has no effect on the observed flux, as expected. In the actual case, $\mathcal{R}$ is close to unity and scattering has only a small effect. Figure B3 shows exp $-\tau_{s}(1-\mathcal{R})$ as a function of the total optical depth for several different cases.

The contribution of the diffuse radiation to the total radiation field obtained by this method can not be directly compared with the estimates obtained from observation because the latter only counts the 
diffuse radiation coming from regions relatively far from sources. For example, the diffuse radiation that originates in lines of sight close to regions of recent star formation such as Gould's Belt are excluded. In $\S 5$, the median value of $F_{\text {scatt }} / F_{\text {tot }}$ from the simulations is about 0.2 whereas the estimates from observations are somewhat less than 0.1 . However, if the scattered light that originates from lines of sight close to the sources are excluded from our calculations, our results are closer to the observations. We find that about $1 / 3$ of the single-scattered radiation has a scattering angle less than 5 deg; omitting such radiation from the "diffuse" category gives a diffuse fraction of about $13 \%$, close to what is observed. 


\section{REFERENCES}

Bakes, E. L. O. \& Tielens, A. A. G. M. 1994, ApJ, 427, 822

Belyaev, V. P., Kurt, V. G., Melioranskii, A. S., Smirnov, A. S., Srokin, L. S. \& Tiit, V. M. 1971, Cosmic Res., 8, 677

Blaauw A. 1961, Bull. Astron. Inst. Netherlands, 15, 265

Blaauw, A. 1964, ARA\&A, 2, 213

Blaauw, A. 1991, in The Physics of Star Formation and Early Stellar Evolution, ed. C. Lada \& N. Kylafis (Dordrecht: Kluwer), 125

Bowyer, S. 1991 ARA\&A 29, 59-88

Bruzual A., G. \& Charlot, S. 1999, ApJ, in preparation

Corbelli, E. \& Salpeter, E. E. 1995, ApJ, 450, 32

de Jong, T. 1977, A\&A, 55, 137

d'Hendecourt, L., \& Léger, A. 1987, A\&A, 180, L9

Dickey, J., \& Lockman, F. J. 1990, ARAA, 28, 215

Draine, B. T. 1978, ApJ SS, 36, 595

Ferriere, K. 1998, ApJ, 503, 700

Gies, D.R. 1987, ApJS, 64, 545

Gies, D.R. \& Bolton, C. T. 1986, ApJS, 61, 419

Gondhalekar, P. M. \& Wilson, R. 1975, A\&A, 38, 329

Gondhalekar, P. M., Phillips, A. P., Wilson, R. 1980, A\&A, 85, 272

Habing, H. J. 1968, Bull. Astr. Inst. Netherlands, 19, 421

Hayakawa, S., Yamashita, K. \& Yoshioka, S. 1969, Ap\&SS, 5, 493

Heiles, C. 1990, ApJ, 354, 483

Henry, R. C., Swandic,J.R., Shulman, S. D. \& Fritz, G. 1977, ApJ, 212, 707

Henry, R. C., Anderson, R. C., Fastie, W. G. 1980, ApJ, 239, 859

Henry, R. C. 1991, ARAA, 29,89

Henyey, L. G. \& Greenstein, J. L. 1941, ApJ, 93,70

Hollenbach, D. J., Parravano, A., \& McKee, C. F. 2002, in preparation.

Hoogerwerf R., de Bruijne J.H.J., and de Zeeuw P.T., 2000, ApJ, 544, L136

Hoogerwerf R., de Bruijne J.H.J., and de Zeeuw P.T., 2000, A\&A, 365, 49

Jura, M. 1974, ApJ, 191, 375

Jura, M. 1976, ApJ, 204, 12

Kaufman, M. J., Wolfire, M. G., Hollenbach, D. J. \& Luhman, M. L. 1999, ApJ, 527, 795 
Leep, S. \& Dalgarno, A. 1988, ApJ, 335, 769

Lejeune,T., Cuisinier, F., \& Buser, R. 1997a, A\&AS, 125, 229

1997b, A\&A, in preparation

Lequeux, L. 1979, A\&A, 80, 35 (L)

Malhotra, S., Kaufman, M. J., Hollenbach, D., Helou, G., Rubin, R. H., Brauher, J., Dale, D., Lu, N. Y., Lord, S., Stacey, G., Contursi, A., Hunter, D. A. \& Dinerstein, H. 2001, ApJ, 561, 766

Massey, P. \& Hunter, D. A. 1998, Apj 493, 180

Massey, P., Johnson, K. E. \& Degioia-Eastwood, K. 1995, ApJ, 454, 151

Matzner, C. D. \& McKee, C. F. 1999, ApJ, 510, 379

McKee, C. F. \& Ostriker, J. P. 1977, ApJ, 218, 148

McKee, C. F. 1989, ApJ, 345, 782

McKee, C. F \& Williams, J. P. 1997, ApJ, 476, 114 (MW97)

Miller, G. E. \& Scalo, J. M. 1979, ApJ, 233, 596 (MS79),

Murthy, J., Henry, R. C., \& Holberg, J. B. 1991, ApJ, 383, 198

Parravano, A. 1988, A\&A, 205, 71

Parravano, A. 1989, ApJ, 347, 812

Parravano, A. \& Mantilla, J. Ch. 1991, A\&A, 250, 70

Poveda, A., Ruiz, J. \& Allen, C. 1967, Bol. Obs. Tonantzintla y Tacubaya, 4, 86

Rocha-Pinto, H. J., Scalo, J., Maciel, W. J., \& Flynn, C. 2000, ApJ, 531, L115

Scalo, J. M. 1986, Fundamentals of Cosmic Physics, 11, 1 (Sc86)

Scalo, J. M. 1998, in The Stellar Initial Mass Function, proceedings of the 38th Herstmonceux Conference, ed. Gary Gilmore and Debbie Howell. ASP Conference Series, Vol. 142, 1998, p.201

Scalo, J., Wheeler, J. C., \& Williams, P. 2001, astro-ph/0104209 \& American Astronomical Society Meeting 198, \#69.09

Stone R. C. 1991, Astron. J, 102, 333.

Tielens, A. G. G. M. \& Hollenbach, D. 1985, ApJ, 291, 722

Vacca. W. D., Garmany, C. D. \& Shull, J. M. 1996, ApJ, 460, 914

Van Buren, D. 1983, Ph.D. Thesis, University Of California, Berkeley (VB)

Verstraete, L., Léger, A., d'Hendecourt, L., Dutuit, O., \& Défourneau. D. 1990, A\&A, 237,436

Watson, W. D. 1972, ApJ, 176, 103

Whitworth, A. 1979, M.N.R.A.S, 186, 59.

Williams, J. P. \& McKee, C. F. 1997, ApJ, 476, 166

Witt, A. N. \& Jonhson, M. W. 1973, ApJ, 181, 363 
Witt, A. N., Gordon, K. D. 1999, ApJ, 528, 799

Wolfire, M. G., Hollenbach, D. J., McKee, C. F., Tielens, A. G. G. M., \& Bakes, E. L. O. 1995, ApJ, 443, 152

Wolfire, M. G., McKee, C. F., Hollenbach, D. J., \& Tielens, A. G. G. M. 2002, ApJ, to be submitted.

Zwicky, F. 1957, Morphological Astronomy (Springer-Verlag, Berlin), 258

This preprint was prepared with the AAS LATEX macros v4.0. 
Fig. 1.- (a) The mean luminosity in the band $[1100-2070 \AA]$ as a function of the stellar mass $m$. The triangles correspond to the calculated mean MS luminosities using stellar evolutionary tracks (see text) and the lines are the eight segment power law fit used to approximate the mass dependence of the luminosity in the simulations. (b) Same as (a) but for the $\mathrm{H}_{2}$ band $[912,1100 \AA]$. (c) The energy output in the FUV band $[912-2070 \AA]$ for a mass distribution of stars following a Present Day Mass Function (PDMF) of MS stars. The thin lines correspond to the case when obscuration is taken into account. (d) Same as (c) but for the $\mathrm{H}_{2}$ band.

Fig. 2.- (a) The mean main sequence ionizing photon production rate $s_{49}$ (units $10^{49}$ photons s${ }^{-1}$ ) as a function of the initial stellar mass. The seven segment power law fit to these data is shown in broken line. (b) The contribution of the different mass ranges to the ionizing photon production rate assuming a Present Day Mass Function of MS stars.

Fig. 3.- Various estimates of the IMF parameters in the plane $\left[\dot{\zeta}_{* h},-\Gamma\right]$ together with three curves of constant total ionizing photon production rate $S_{T, 53}=S_{T} /\left(10^{53}\right.$ photons s$\left.^{-1}\right)$. The letters beside the x symbols labels the estimates of Lequeux 1979 (L), Miller \& Scalo 1979 (MS), Van Buren 1983 (VB, b, c), and Scalo 1986 (Sc86). The 90\% and 99\% confidence contours correspond to Van Buren data. The cross near the label $\mathrm{P}$ indicates the adopted values of $\dot{\zeta}_{* h}$ and $\Gamma$ in the present work.

Fig. 4.- Evolution of the FUV luminosity for associations that ultimately form 1000, 100, and 10 high-mass stars in five generations. The contribution by a single association to the energy density $u_{\mathrm{FUV}}\left(u_{-17}=u / 10^{-17} \mathrm{erg} \mathrm{cm}^{-3} \AA^{-1}\right)$ at a point located $100 \mathrm{pc}$ away, assuming that there is no extinction, is indicated in the right vertical axis.

Fig. 5.- The projection on the plane of the positions of associations born in a period of $500 \mathrm{Myr}$ (left panel) and $30 \mathrm{Myr}$ (right panel). Large pentagons, large stars, small stars, and dots correspond respectively to associations with sizes characterized by $\mathcal{N}_{*, h}$ stars with $1000<\mathcal{N}_{*, h}<\mathcal{N}_{* h, u}$, $100<\mathcal{N}_{*, h}<1000,10<\mathcal{N}_{*, h}<100$, and $\mathcal{N}_{* h, l}<\mathcal{N}_{*, h}<10$. The right panel reflects the OB associations, since the OB stars die after about $30 \mathrm{Myr}$.

Fig. 6. - Expected increase of the mean energy density $\left\langle U_{\mathrm{FUV}}\right\rangle_{t_{\mathrm{sim}}}$ in the simulation time interval $\left[0, t_{\mathrm{sim}}\right]$ as $t_{\mathrm{sim}}$ increases. Values normalized to the asymptotic mean value $\left\langle U_{\mathrm{FUV}}\right\rangle_{\infty}$.

Fig. 7.- Time evolution of the FUV energy density at a point located at the center of simulation area and in the galactic plane. The bars in between the arrows indicate the periods of time where the point is inside an HII region; the labels give the characteristics of the association that produces the HII region: size $\mathcal{N}_{*, h}$, distance $r$, ionizing luminosity $s_{49}$ and the mass $m_{1}$ of the most massive star.

Fig. 8. - (a) Time evolution of the energy density in the $[1100-2070 \AA]$ band $U_{\mathrm{FUV}-\mathrm{H}_{2}}$ (continuous line) and in the $[912-1100 \AA] \mathrm{H}_{2}$ band $U_{\mathrm{H}_{2}}$ (dotted line). (b) Ratio of the energy density in the $[912-1100 \AA]$ band, $U_{\mathrm{H}_{2}}$, to the energy density in the $[1100-2070 \AA]$ band, $U_{\mathrm{FUV}-\mathrm{H}_{2}}$, as a function of the FUV energy density $U_{\mathrm{FUV}}$. Each data point corresponds to the radiation field every 20 time 
steps (i.e. $20 \Delta t_{a} \sim 1 \mathrm{Myr}$ ) and the time period plotted is from $t_{\text {sim }}=1000$ to 4600 Myrs (see Fig 7). (c) The fraction of $U$ that comes to the observer as scattered light as function of $U_{-17}$ in the $1100-2070 \AA$ band. The times are the same as in Figure 8b. (d) The fraction of $U$ in the FUV band that comes to the observer from the dominant source as function of $U_{-17}$ in the FUV band.

Fig. 9.- (a) Distribution of the occurrence of $U_{\mathrm{H}_{2}}$ values (dashed) and of $U_{\mathrm{FUV}}$ values (nondashed). The arrows indicate the mean and median values of the distributions (see text $\S 4.2$ ). (b) Probability $P(>U)$ that, at a given instant in the simulation, the FUV energy density is greater than $U$. The curves labeled $1 \mathrm{Gyr}, 4 \mathrm{Gyr}$, and $56 \mathrm{Gyr}$, correspond respectively to the probabilities $P(>U)$ calculated considering only the evolution of $U$ in the time intervals $\Delta t=1 \mathrm{Gyrs}, 4 \mathrm{Gyr}$, and 56 Gyr. The negative slope dashed line shows a power law fit to $P(>U)$ for large values of $U$. The positive slope dashed line shows the corresponding power law for the typical time $t_{U}$ (right vertical axis) between two events that raise the energy density to $>U$ at a fixed point in space.

Fig. 10.- Comparison of the evolution of mean energy density $\langle U\rangle_{t_{\mathrm{sim}}}$ in a single simulation of time interval $t_{\mathrm{sim}}$ and the expected relation (see Figure 6) of a large number of simulations. The corresponding evolution of the median value for a single simulation is also shown. Values normalized to the asymptotic mean value $\left\langle U_{\mathrm{FUV}}\right\rangle_{\infty}$. See text $(\S 4.2)$.

Fig. 11. - Evolution of the surface density of SN progenitors $\varsigma_{* h}$ in the simulation. The continuous line corresponds to the average surface density over $A_{\text {sim }}=5 \mathrm{kpc}^{2}$ and the dotted line corresponds to the average within 500 pc of the central point.

Fig. 12.- $\Delta U_{-17 \text {,run }}\left(t_{\text {sim }}\right)$ (heavy line) and the total energy density $U_{\mathrm{FUV}}$ from the simulation (dotted line). The labels at the top give the properties of the runaway stars producing the most prominent FUV spikes (i.e. the stellar mass $m$, the velocity of ejection $v_{\text {ejec }}$, the distance of closest approach $r_{\min }$ to the point in question, and the generation in which the runaway was produced in the association). The labels at the bottom give the properties of the association where the runaway was born (i.e. the size of the association $\mathcal{N}_{* h}$ and its distance to the observer $r_{\text {form }}$ ).

Fig. 13.- Fig. B1.- Geometrical parameters of the scattering problem.

Fig. 14.- Fig. B2.- $\mathcal{R}$ as a function of the distance $r_{o e}$ for $z_{e}$ and $z_{o}$ at the mid plane (continuous line), $z_{o}=z_{e}=100 \mathrm{pc}$ (long dash line), and $z_{o}=100 \mathrm{pc}$ and $z_{e}=-100 \mathrm{pc}$ (short dash line).

Fig. 15.- Fig. B3.- Ratio of total flux to the flux for pure absobtion $\exp -\tau_{s}(1-\mathcal{R})$ as a function of the total optical depth for the three cases in Fig. B2. 


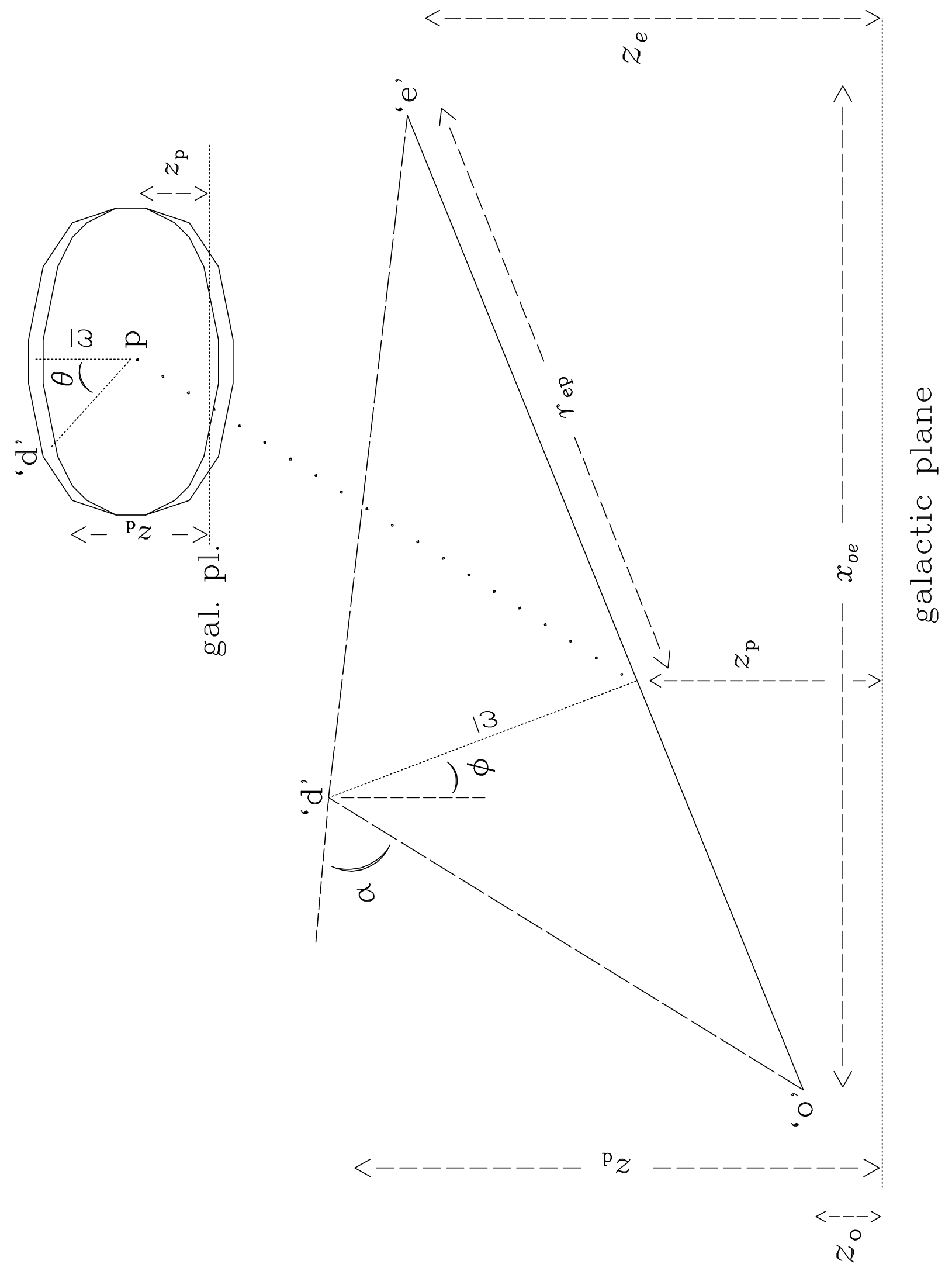




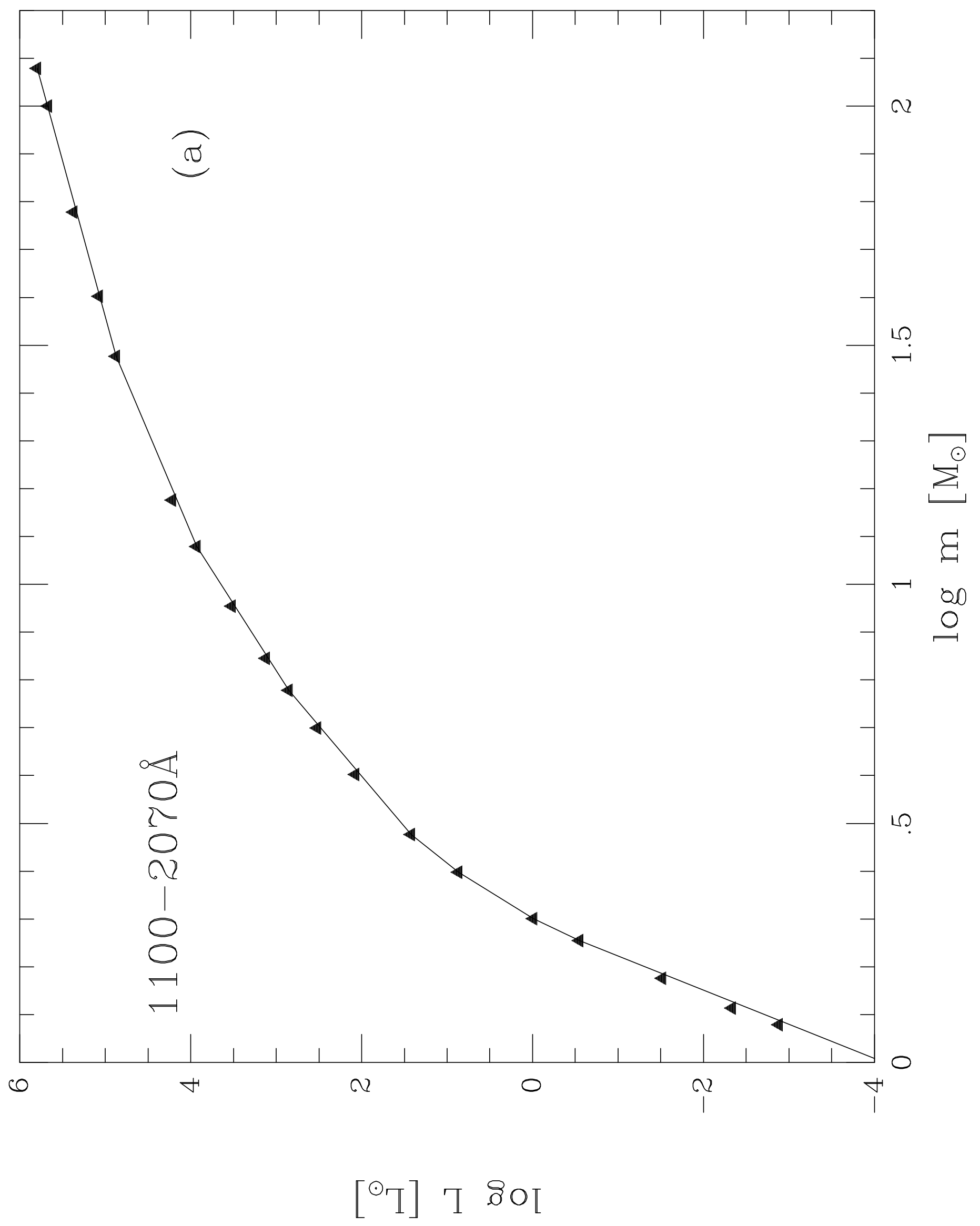




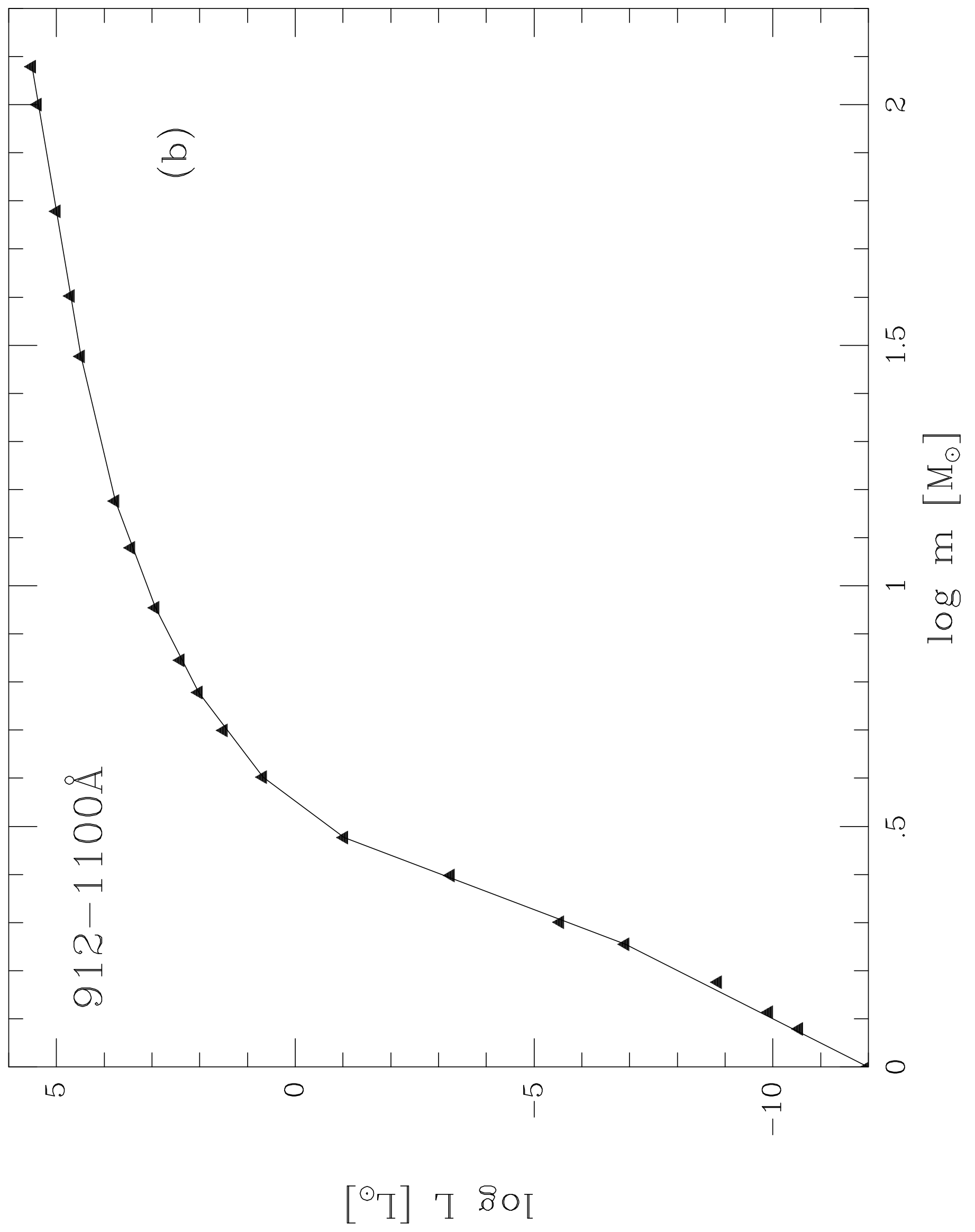




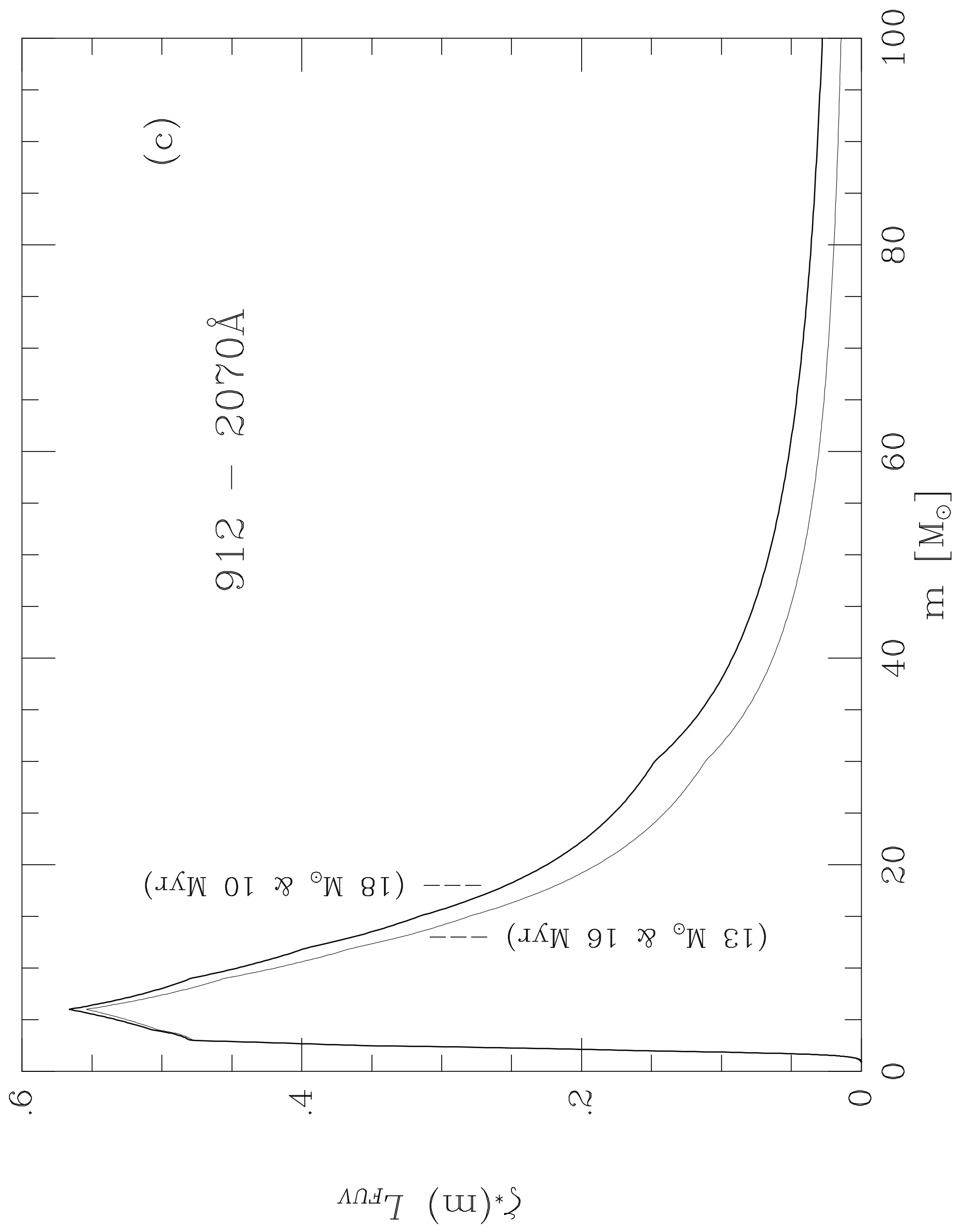




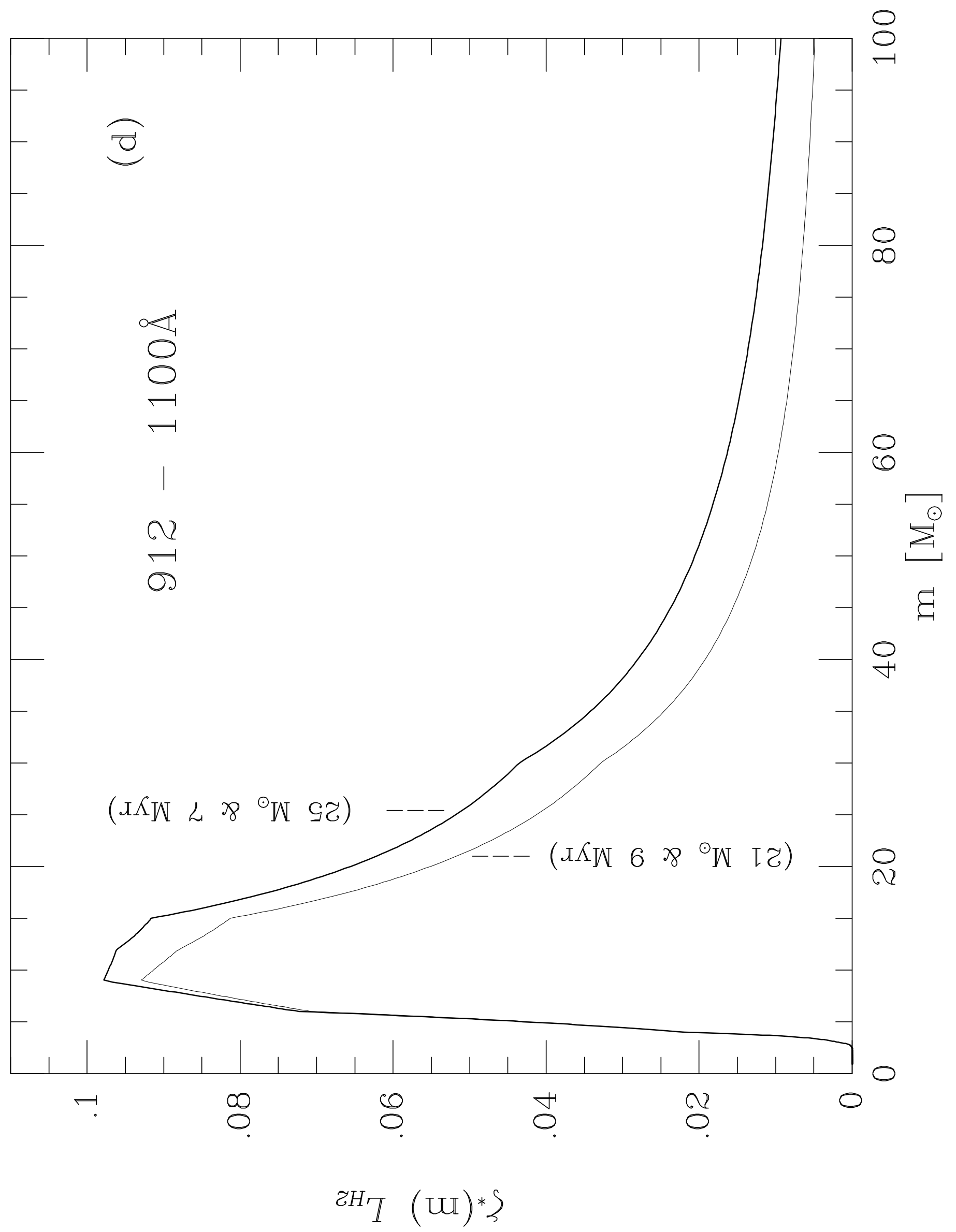




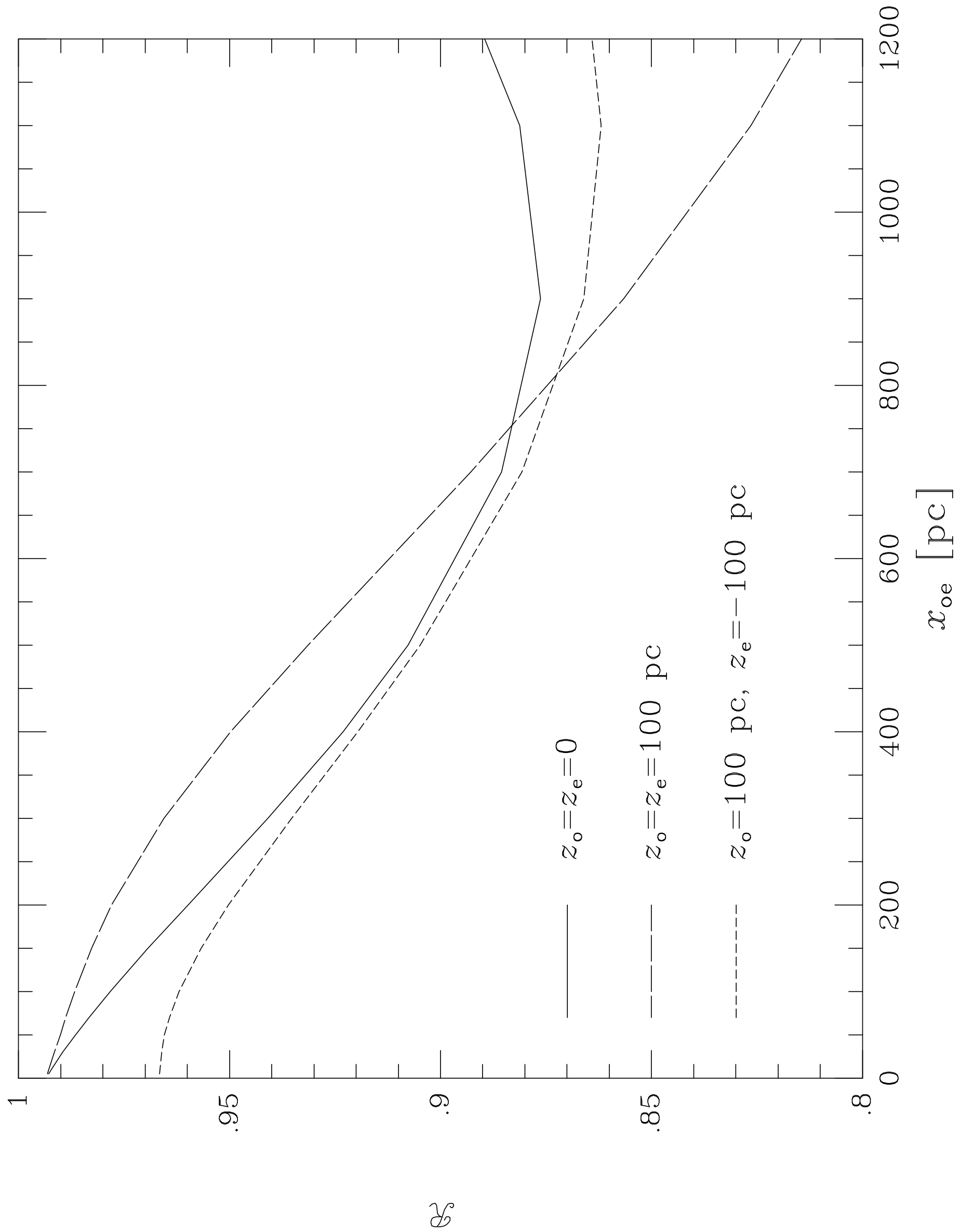




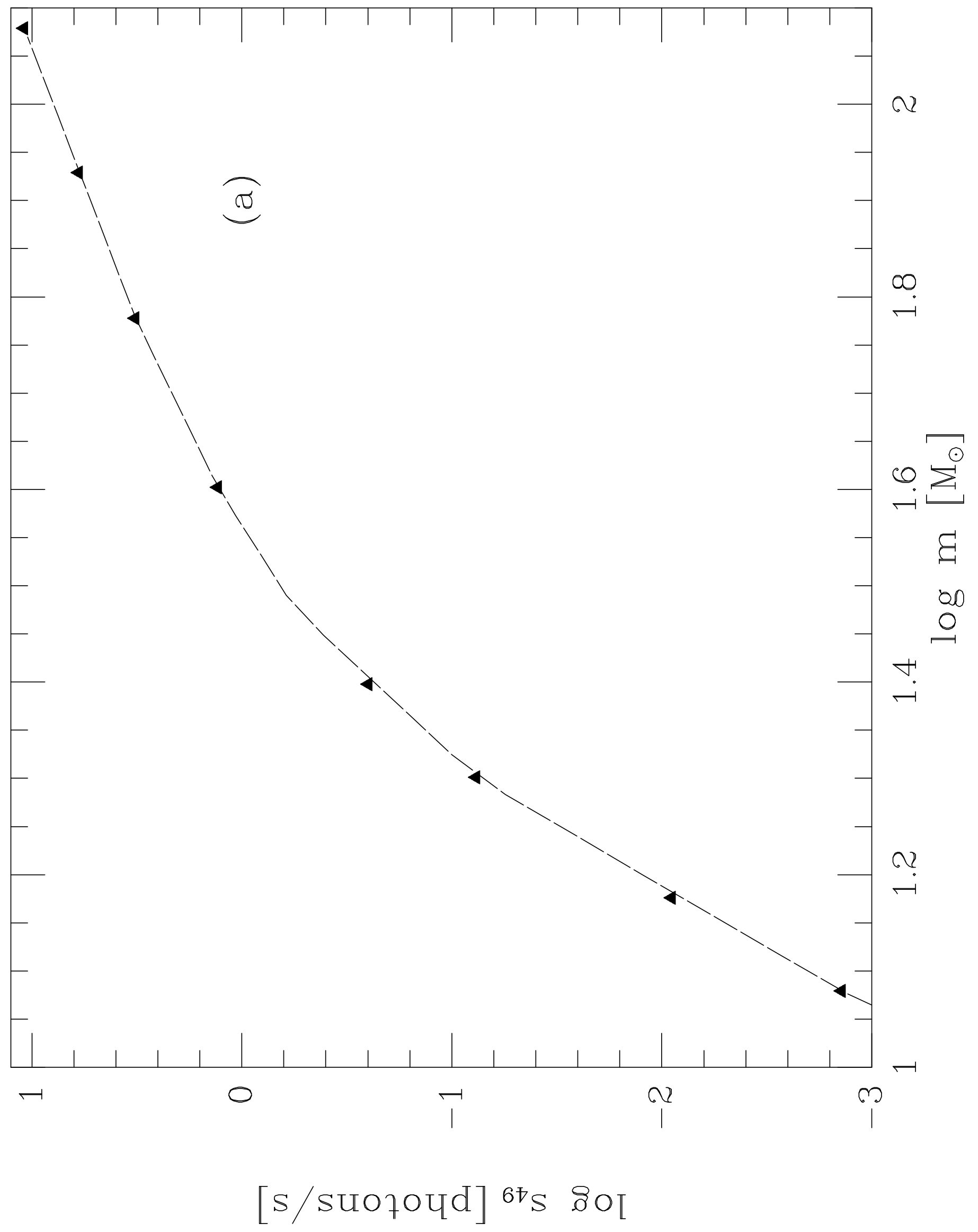




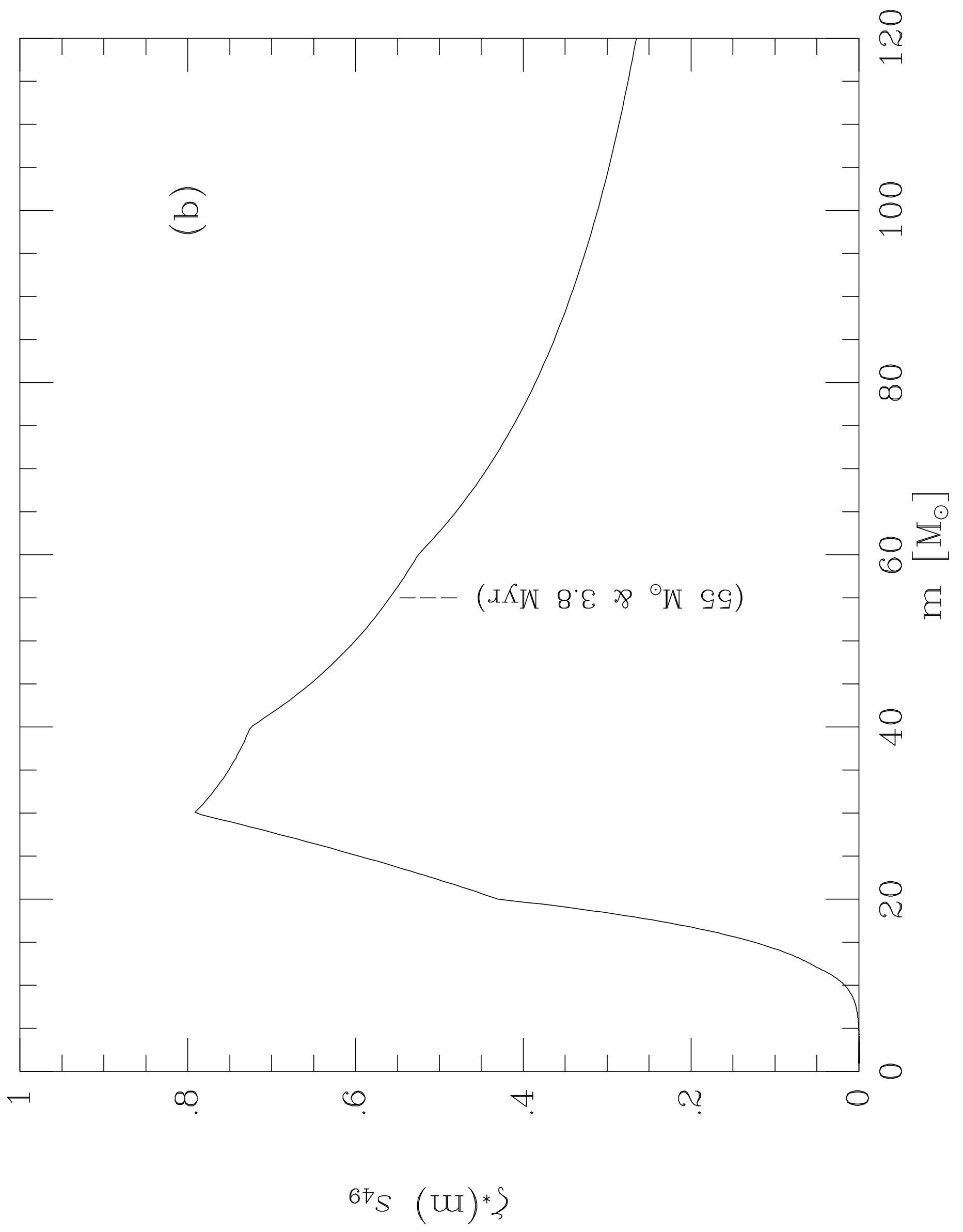




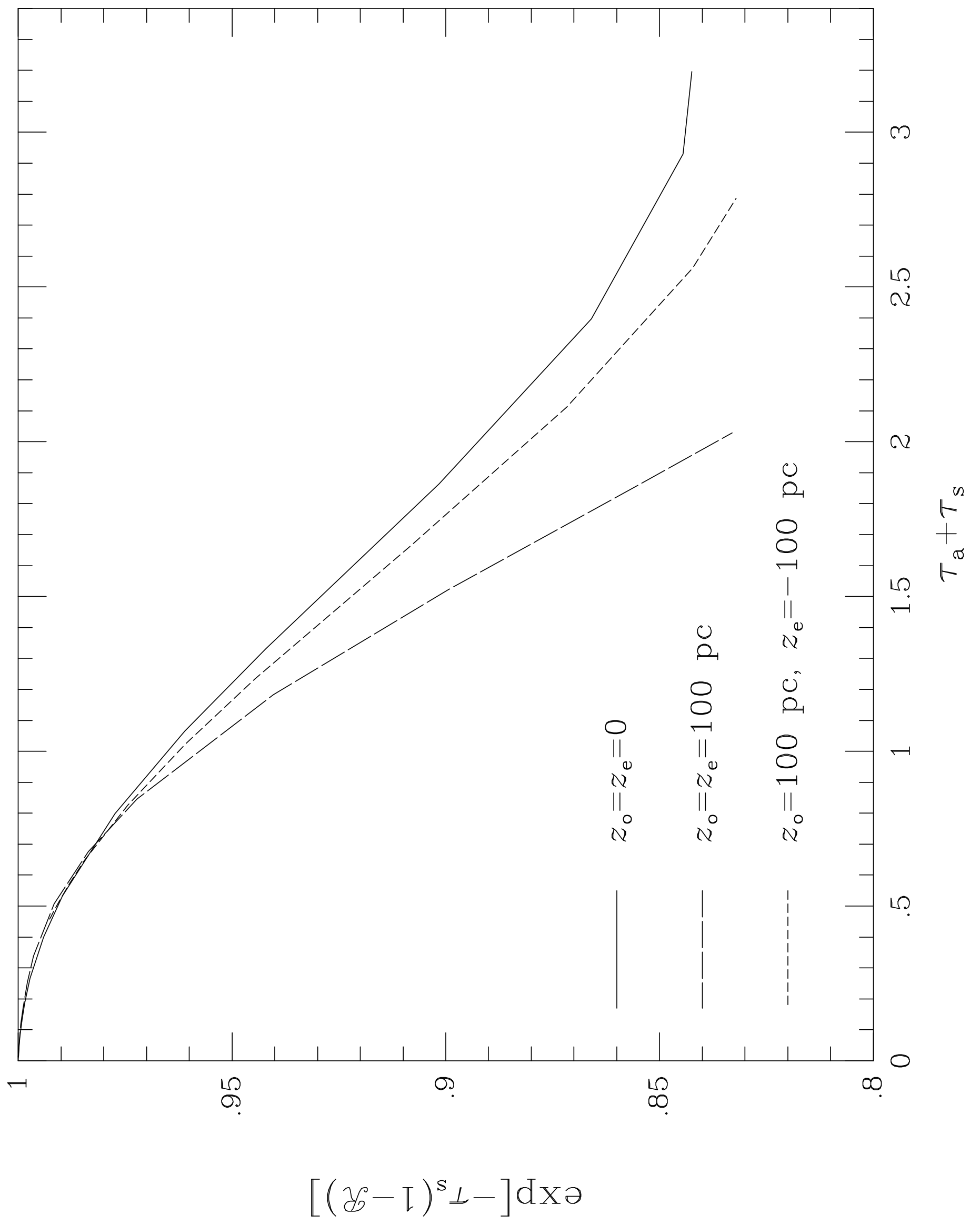




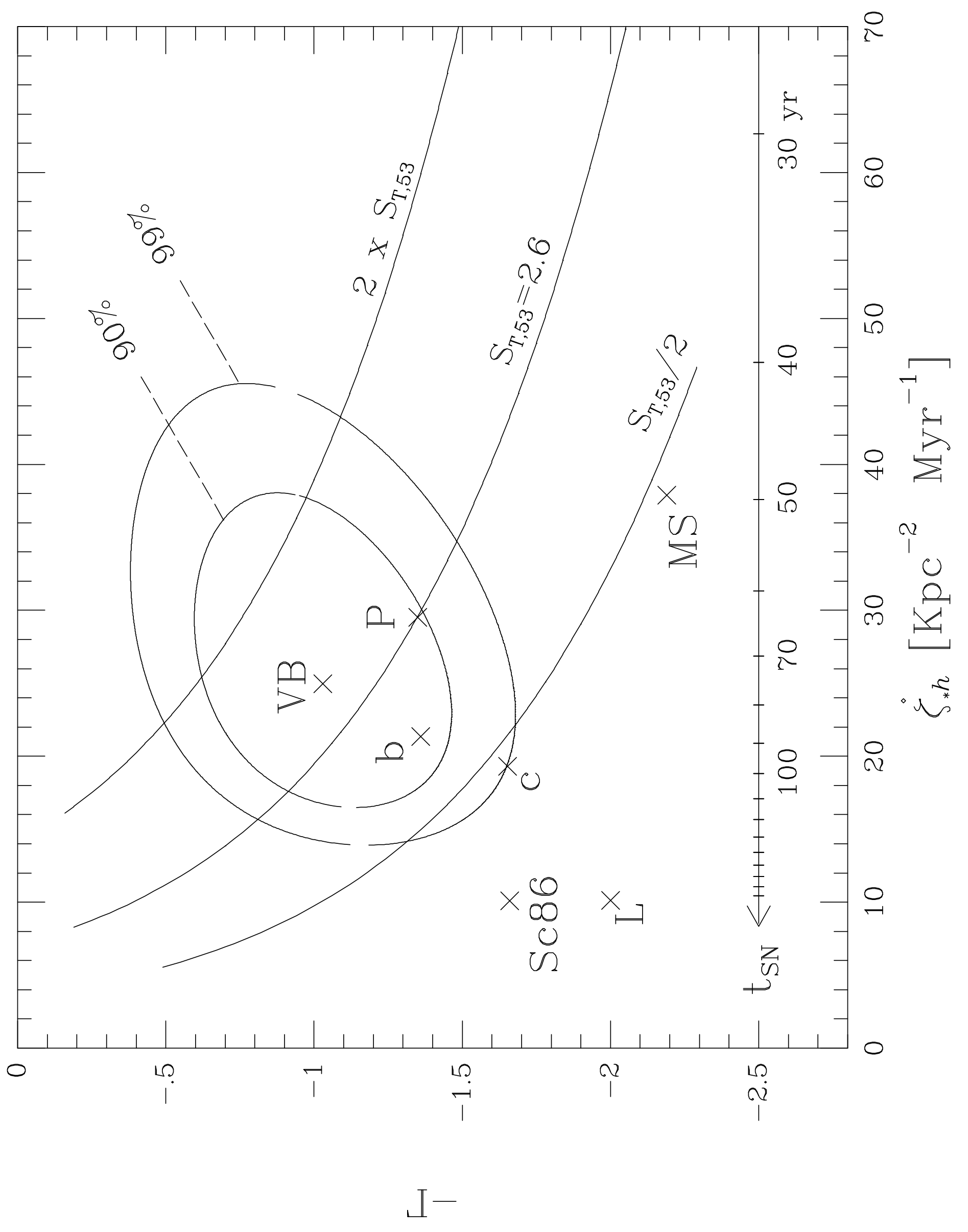




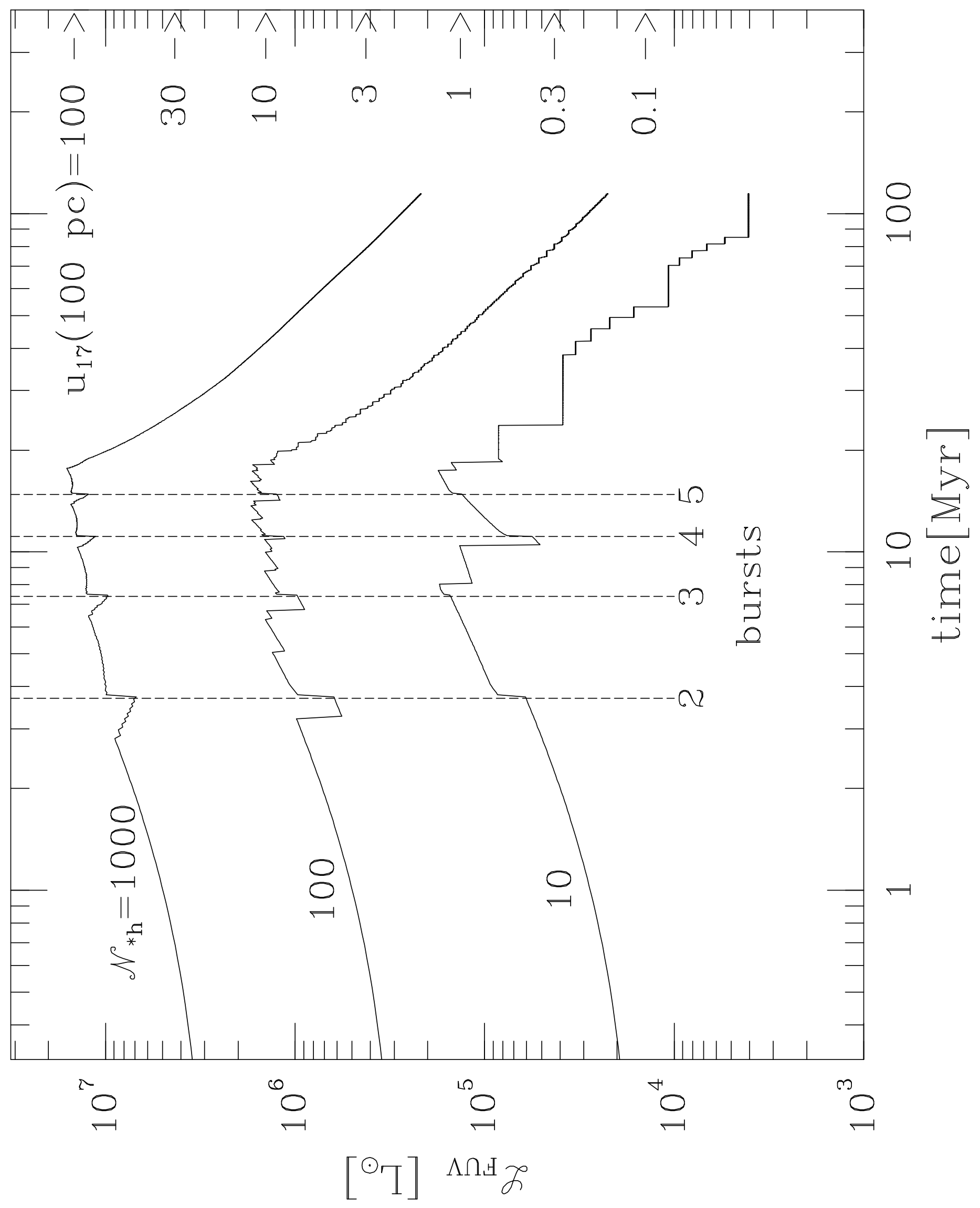



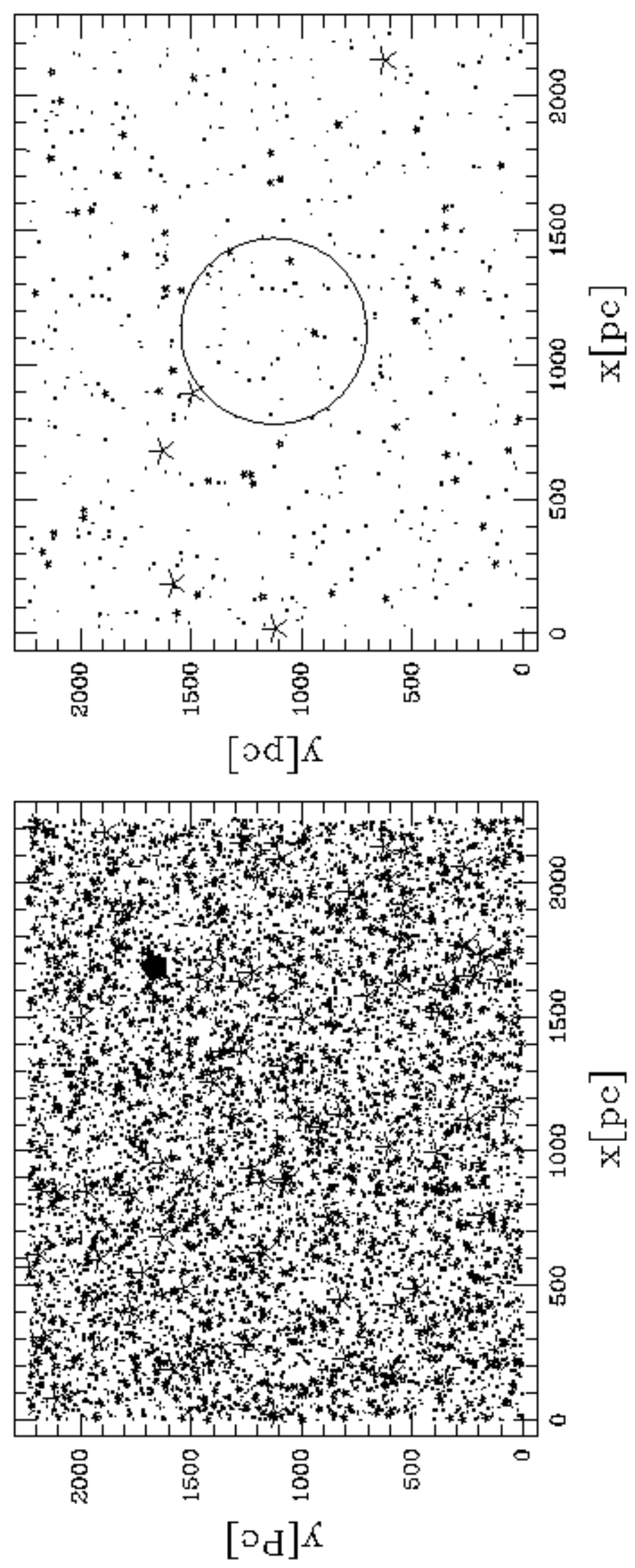


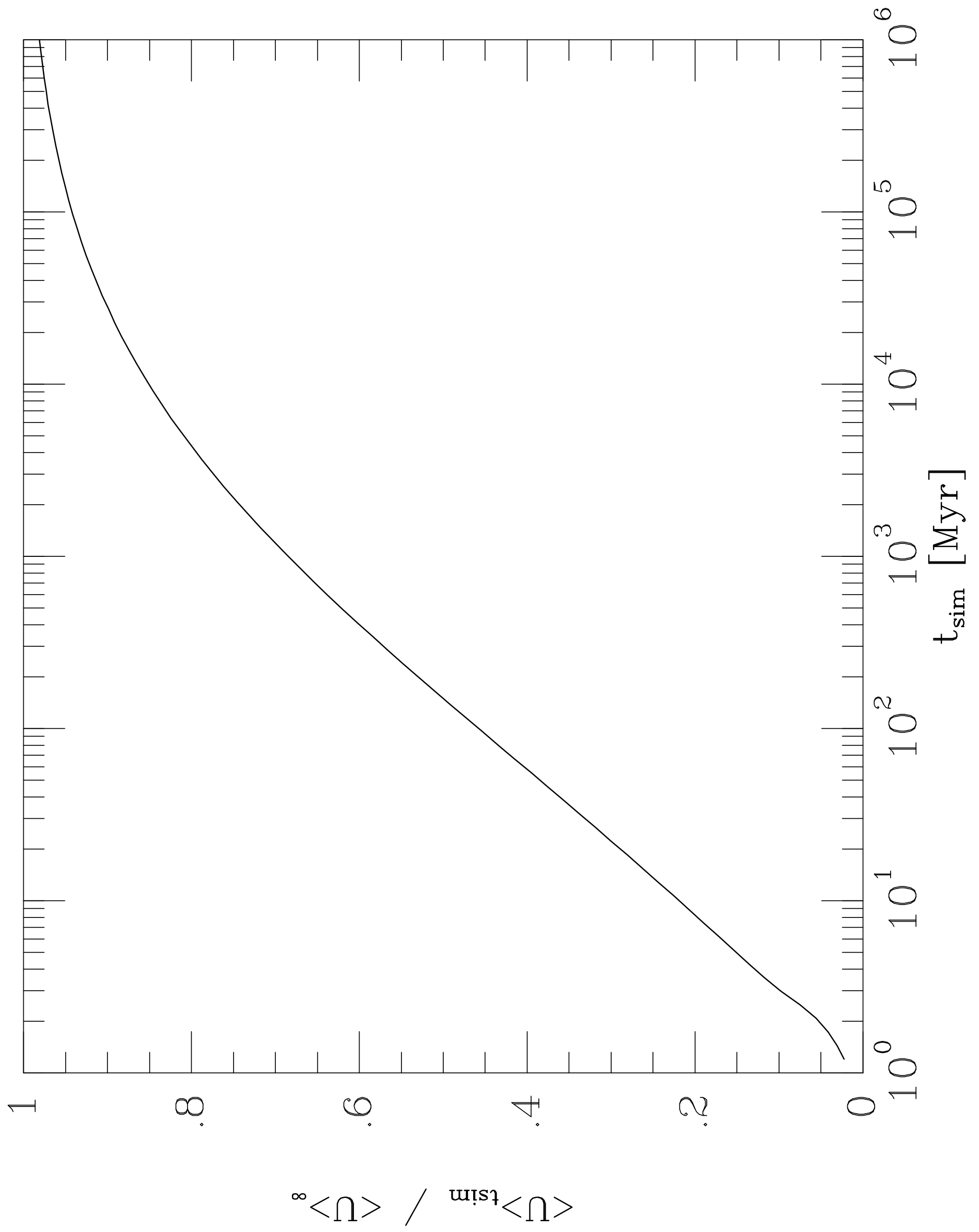




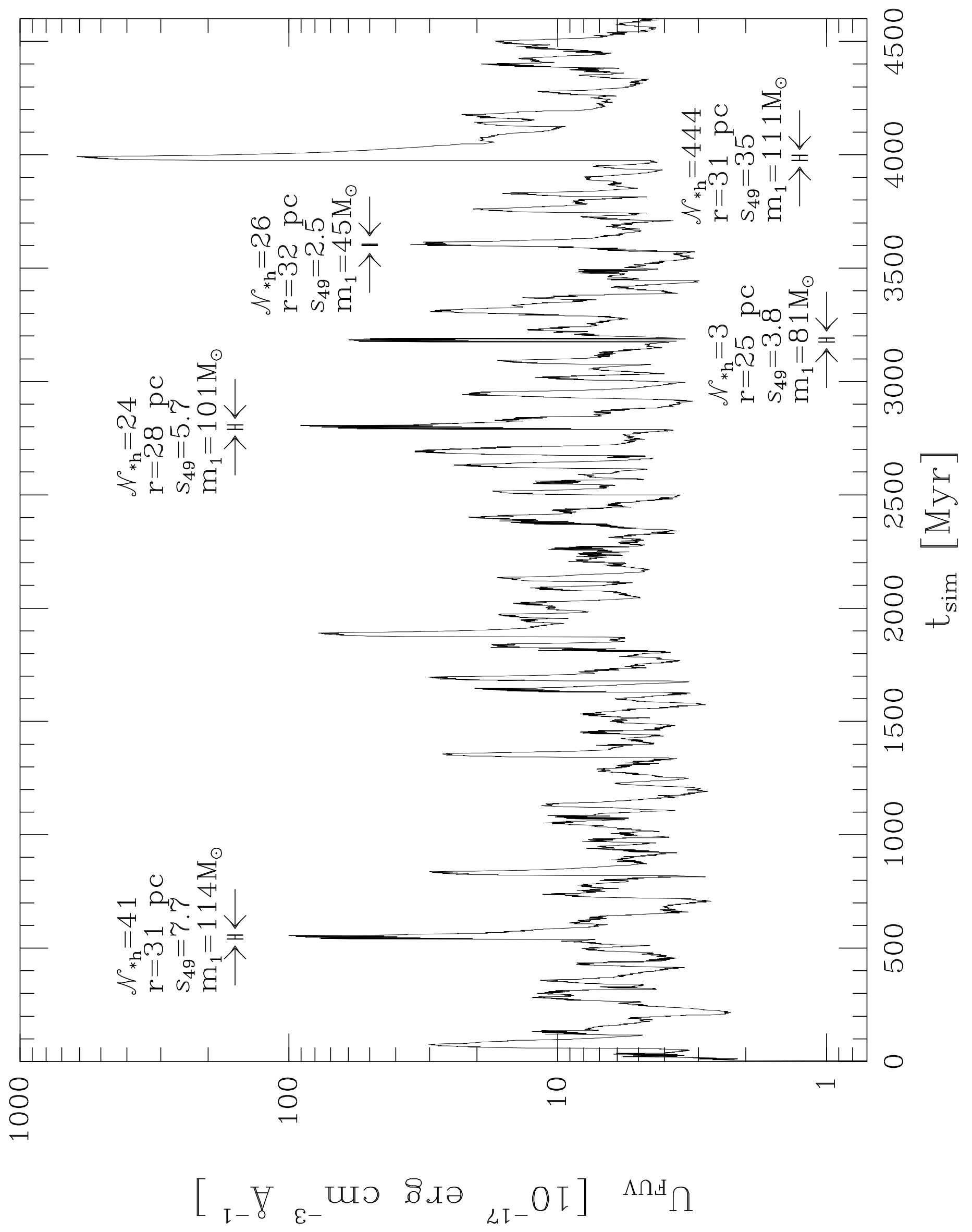




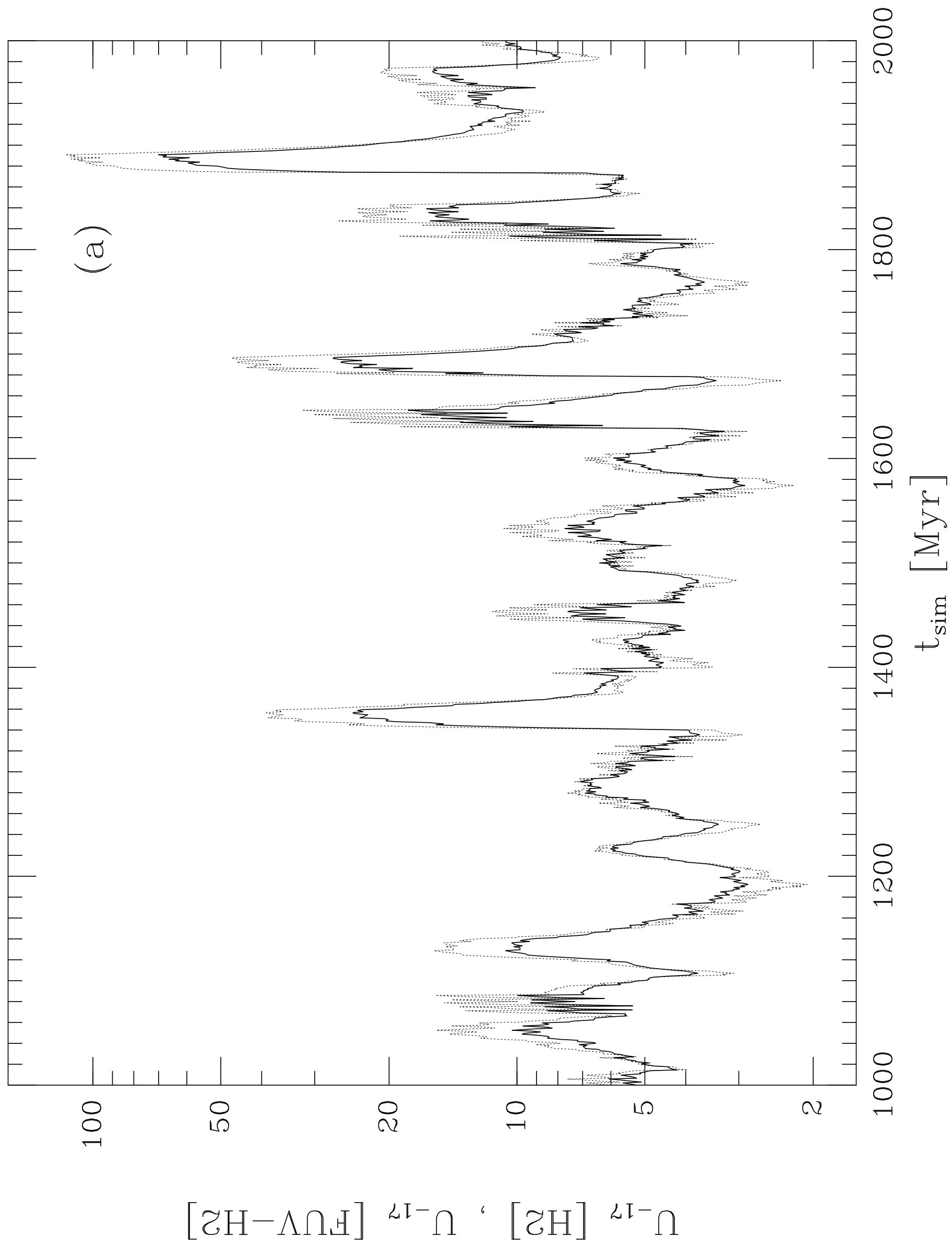




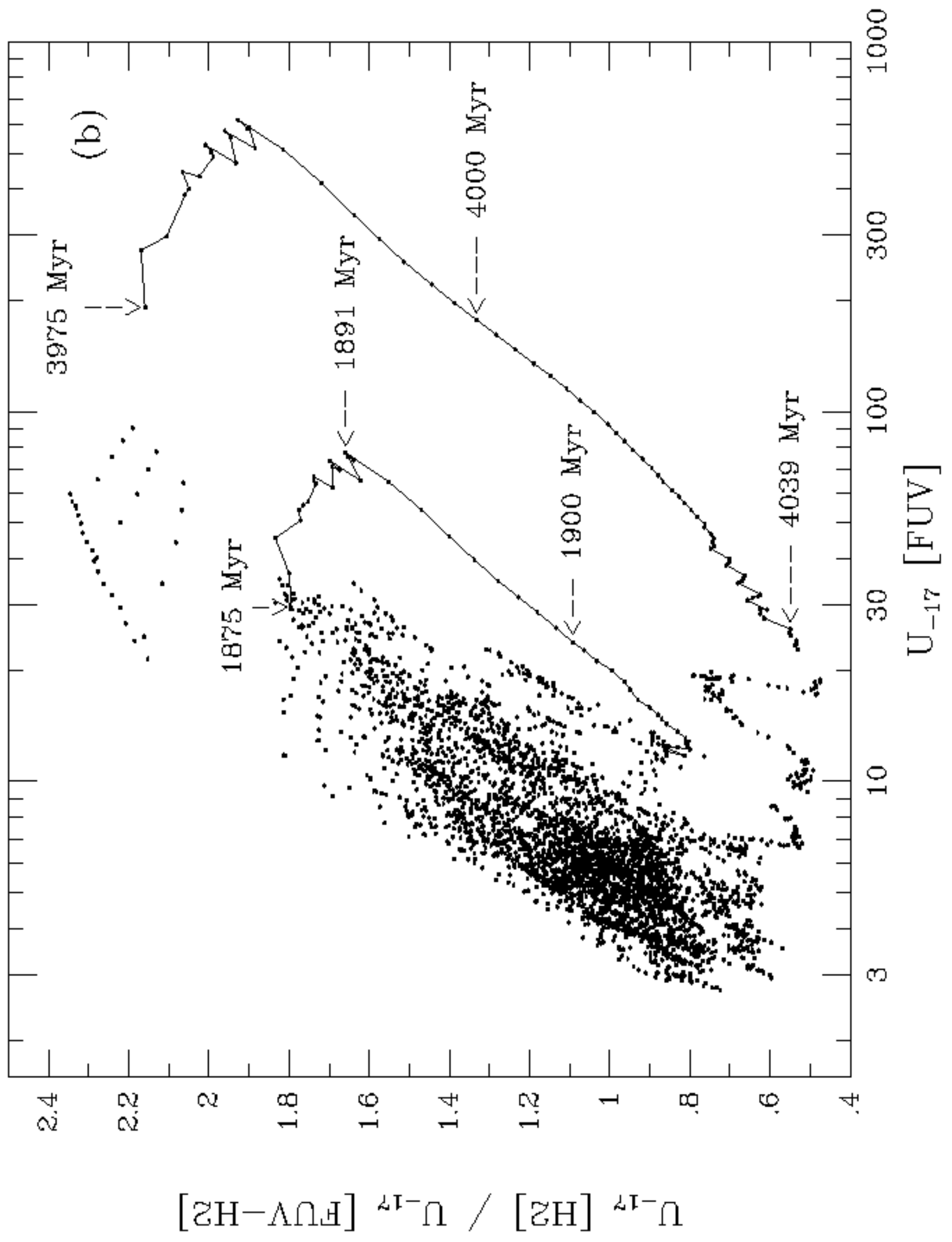




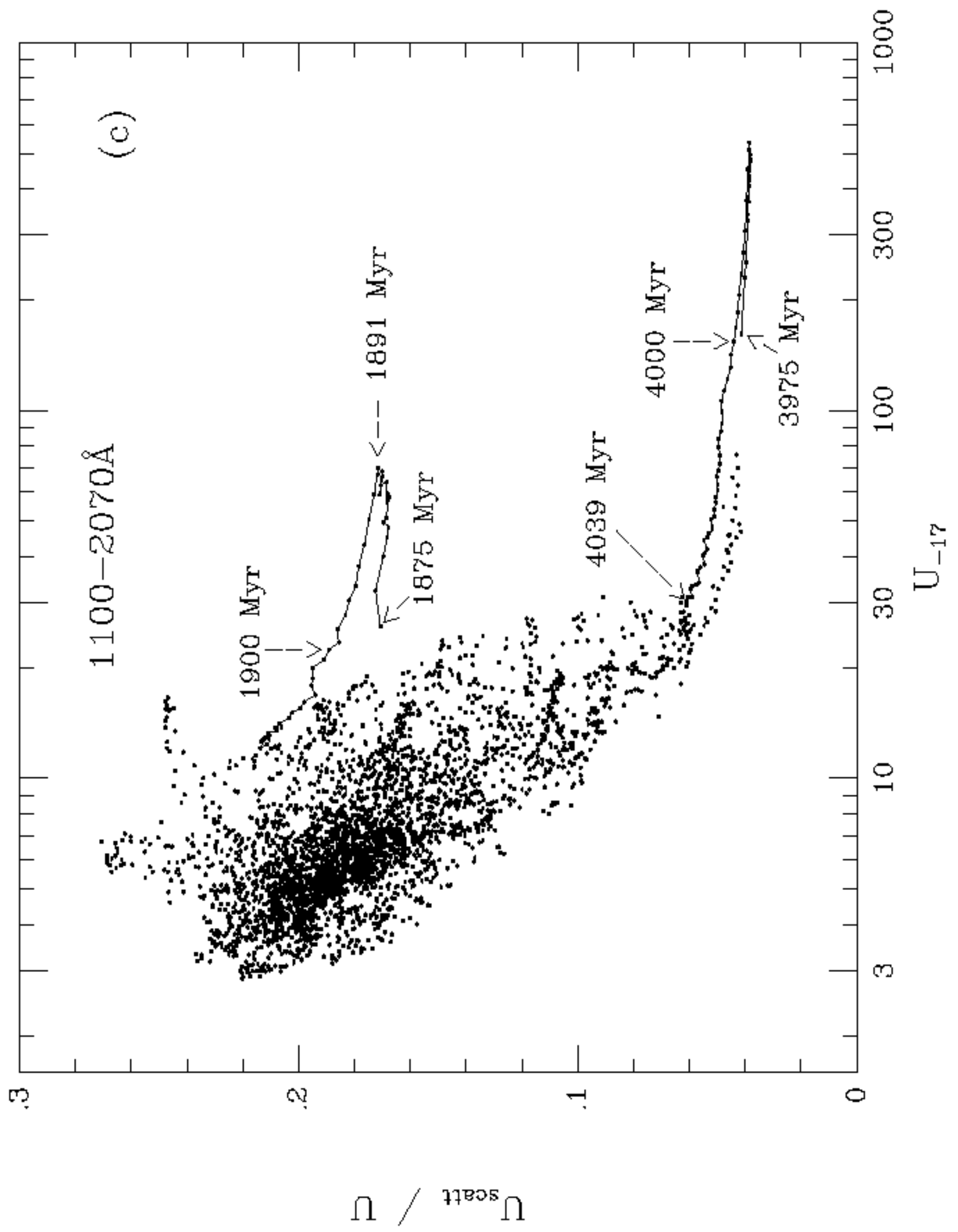




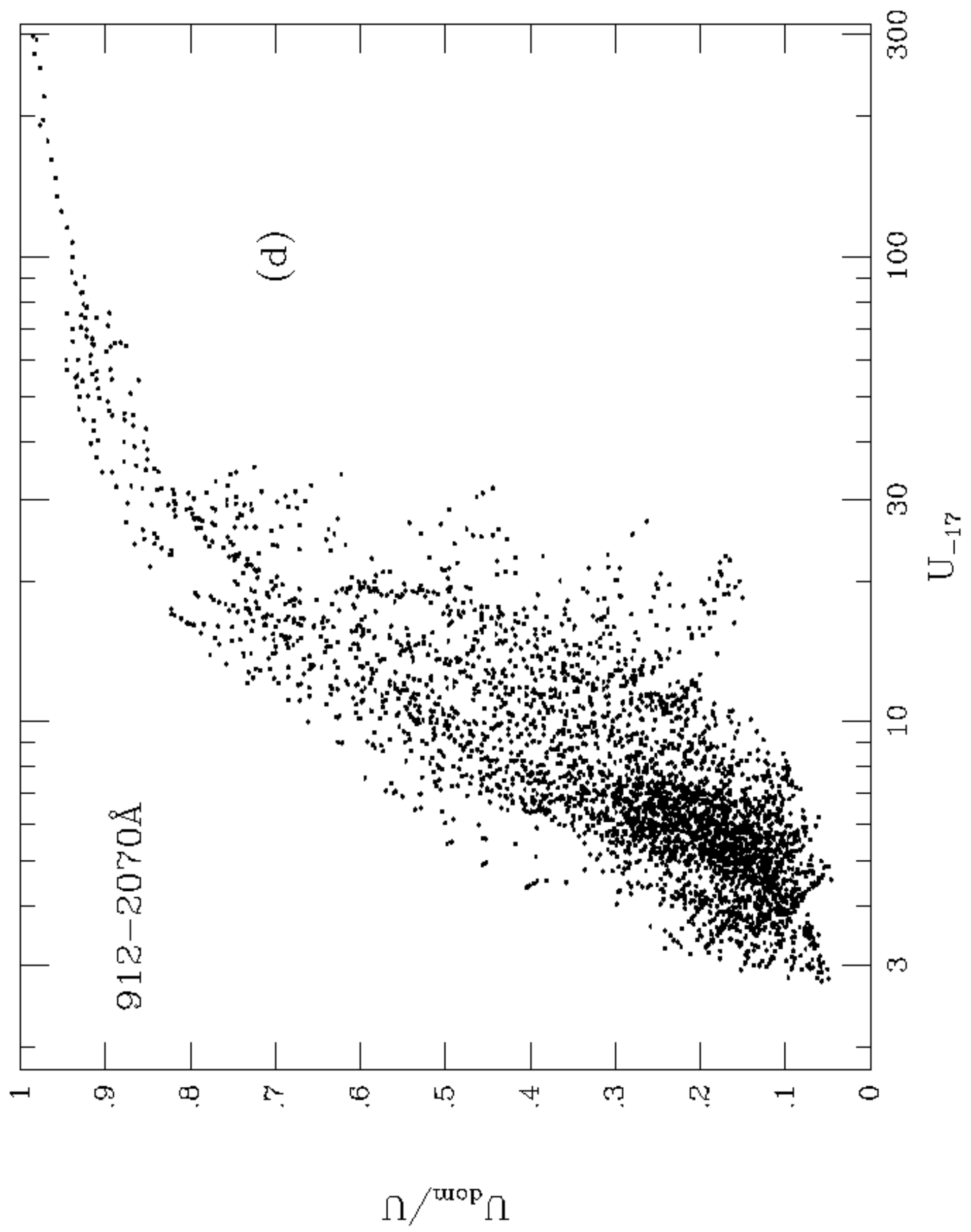




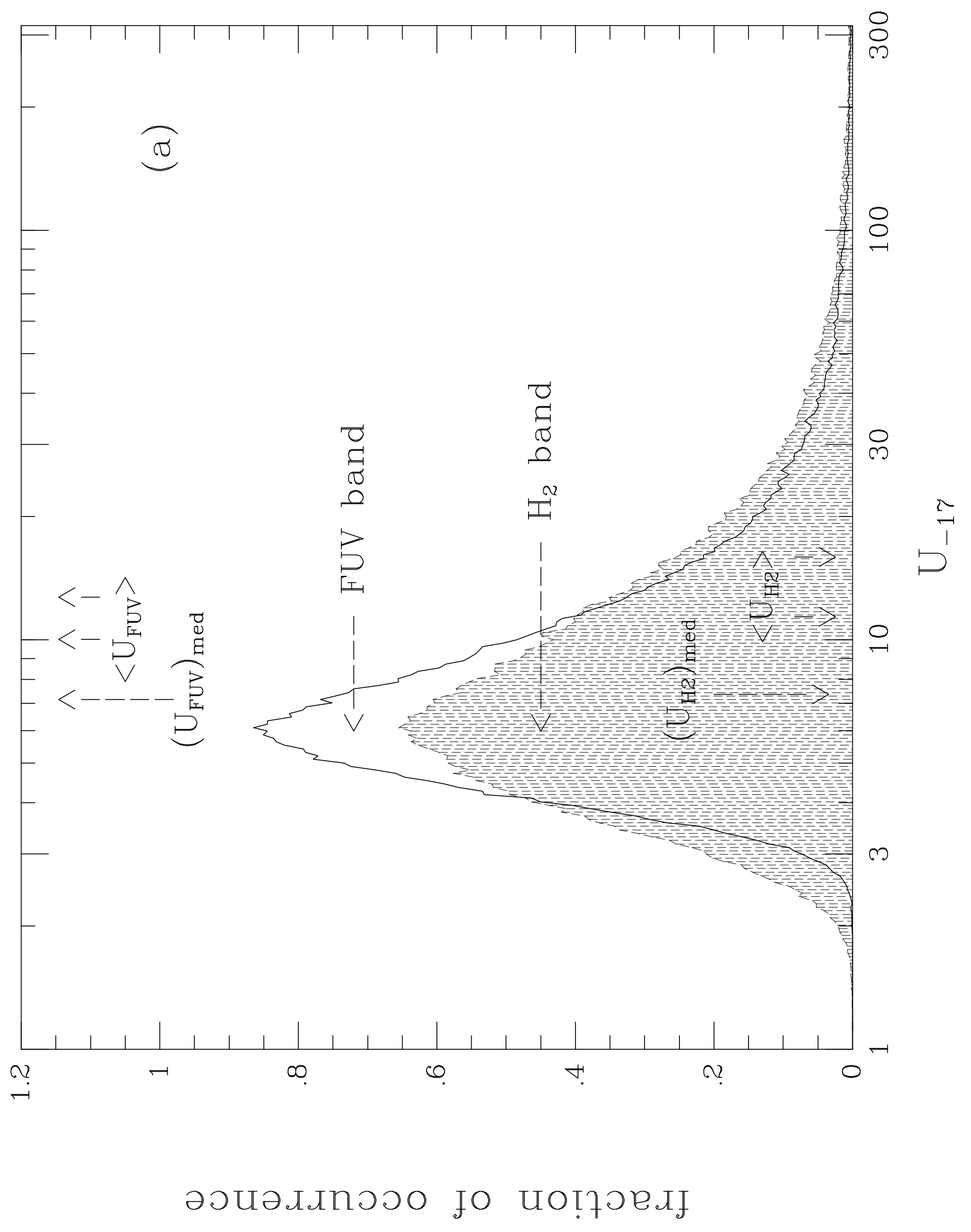




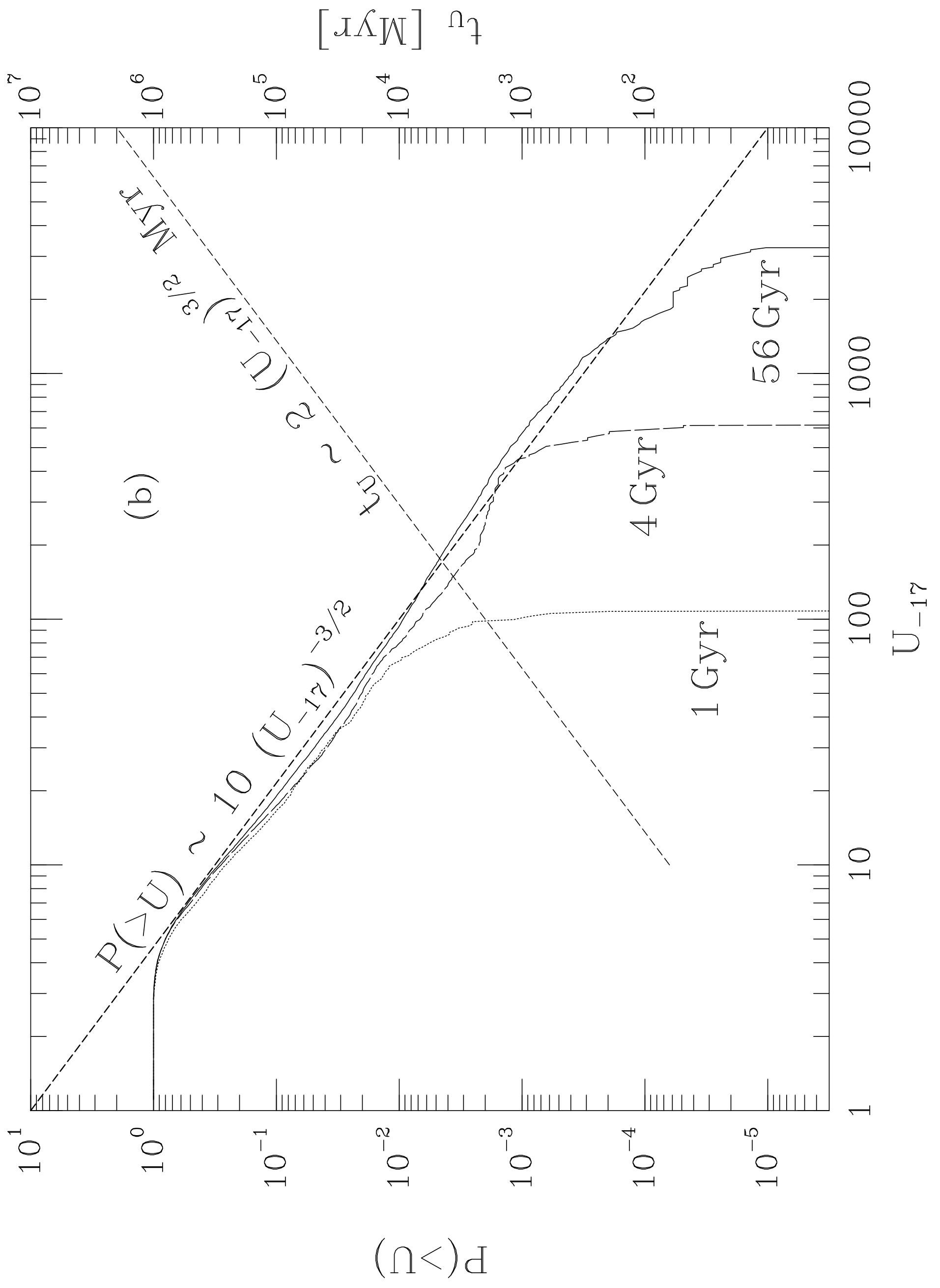




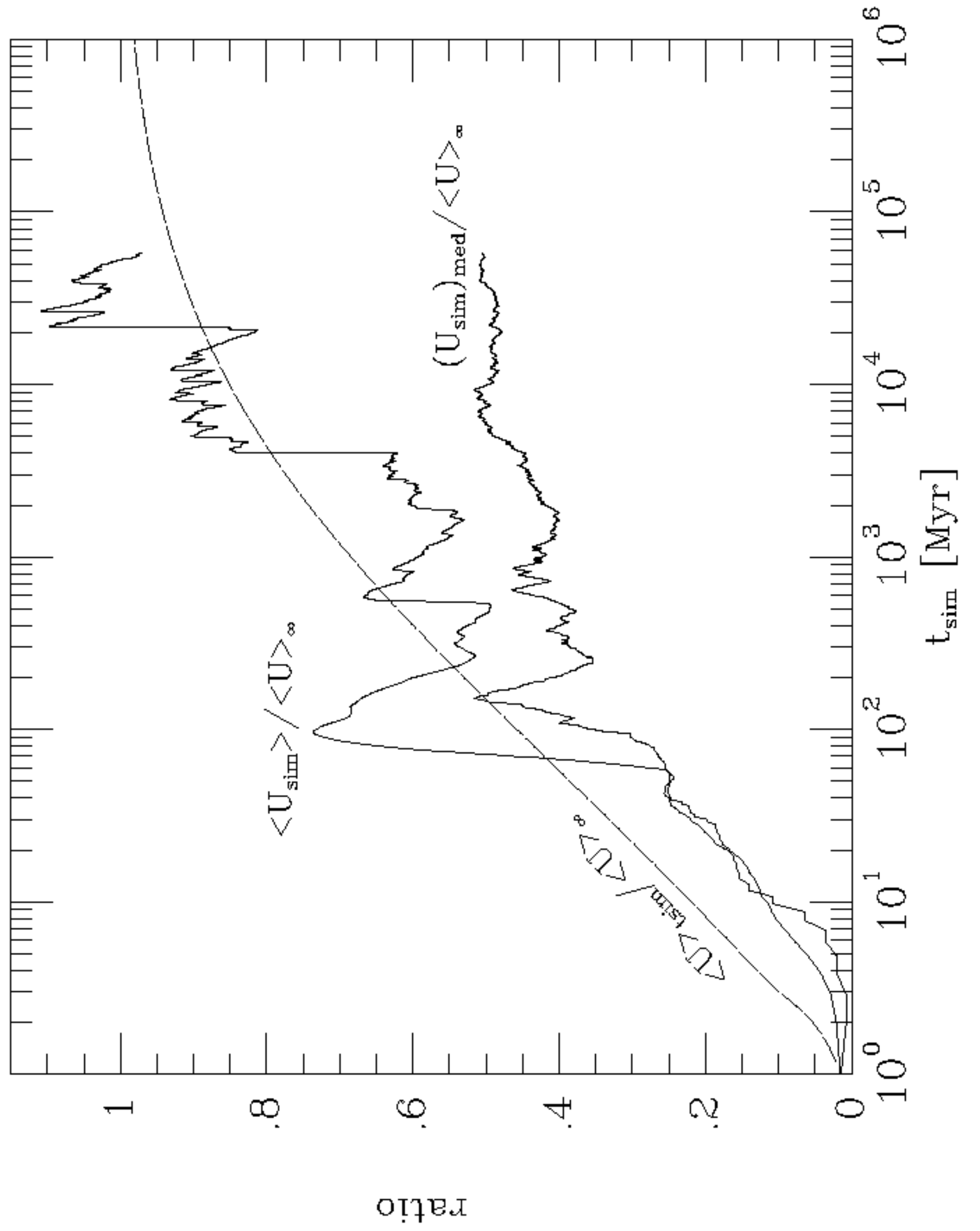




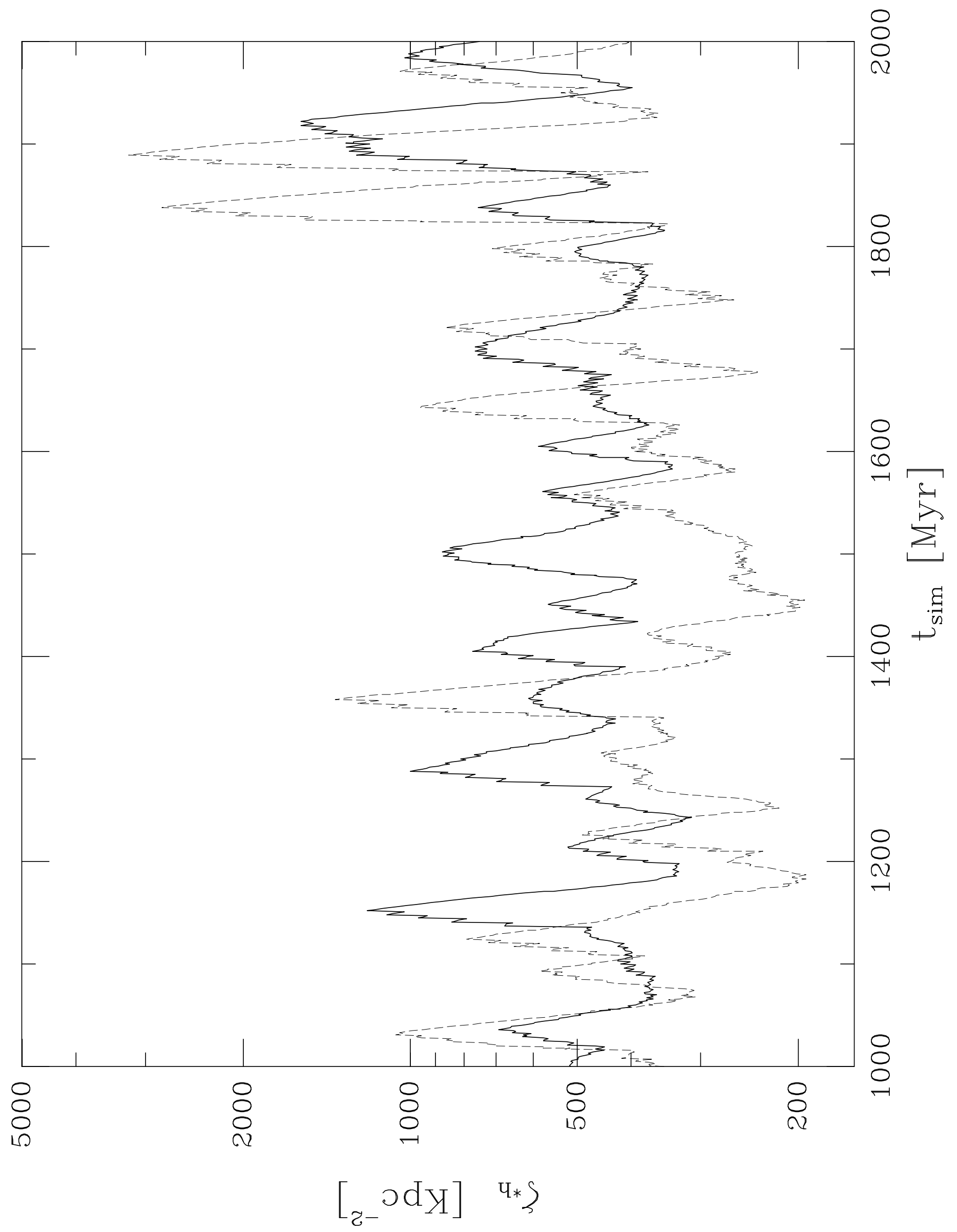




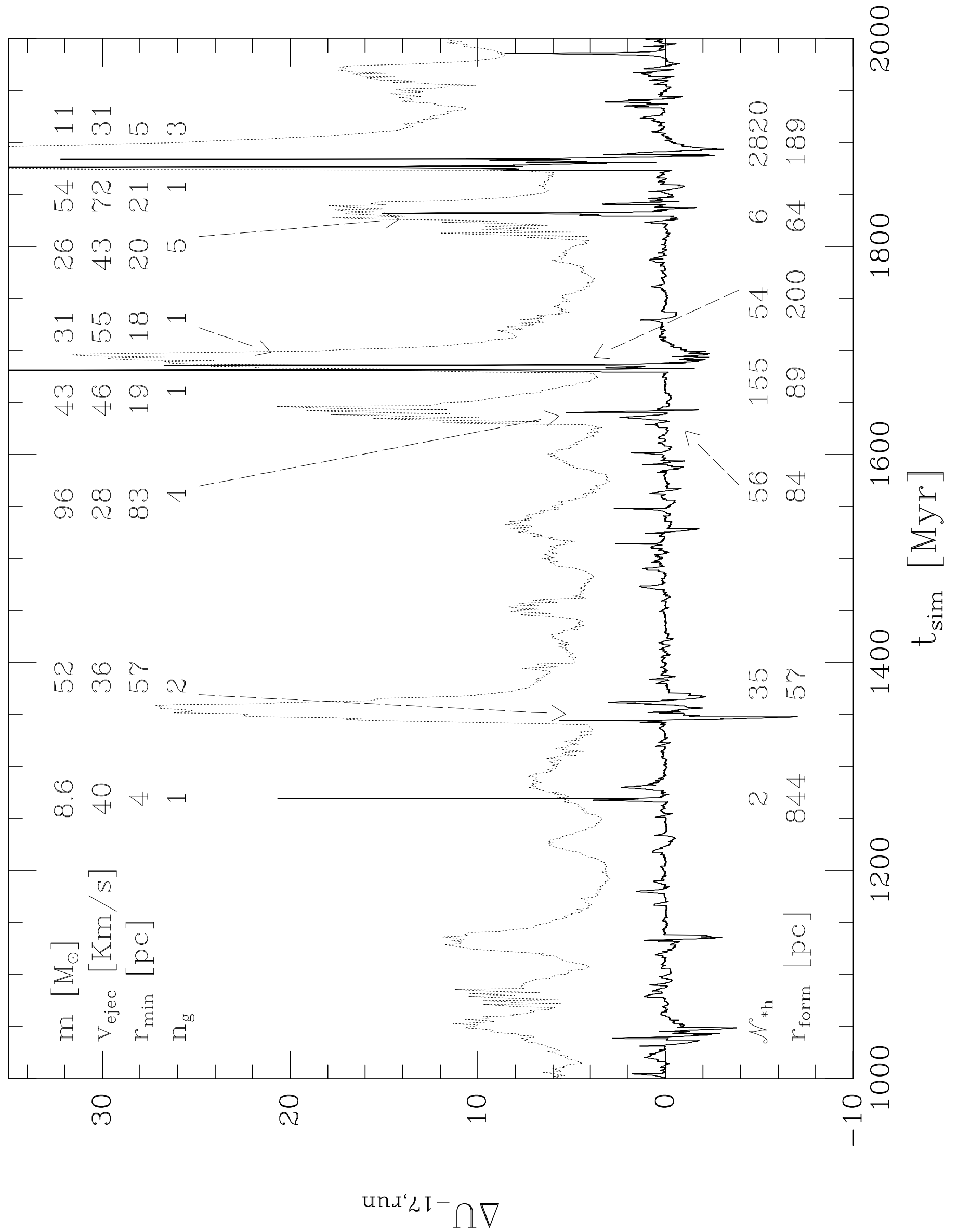

